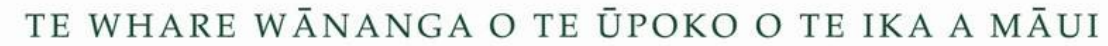

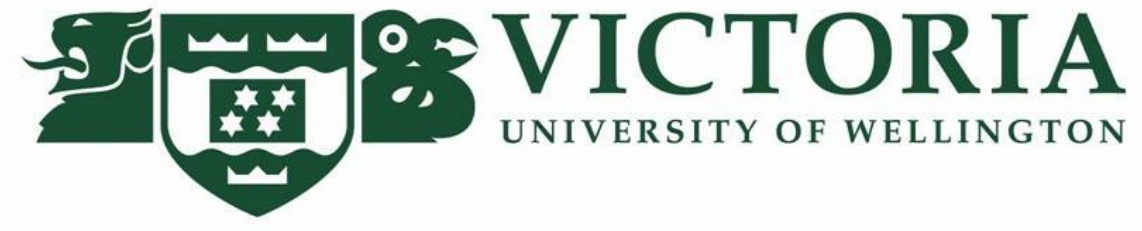

\title{
Characterising Radio Emissions in Cosmic Filaments
}

\author{
Rowan Miller
}

Supervisor: Dr. Melanie Johnston-Hollitt 


\begin{abstract}
A growing number of radio studies probe galaxy clusters into the low-power regime in which star formation is the dominant source of radio emission. However, at the time of writing no comparably deep observations have focused exclusively on the radio populations of cosmic filaments. This thesis describes the ATCA $2.1 \mathrm{GHz}$ observations and subsequent analysis of two such regions - labelled Zone 1 (between clusters A3158 and A3125/A3128) and Zone 2 (between A3135 and A3145) - in the Horologium-Reticulum Supercluster (HRS). Source count profiles of both populations are discussed and a radio luminosity function for Zone 1 is generated. While the source counts of Zone 2 appear to be consistent with expected values, Zone 1 exhibits an excess of counts across a wide flux range $\left(1 \mathrm{mJy}<\mathrm{S}_{1.4}<200 \mathrm{mJy}\right)$. An excess in radio activity at the lower extent of this range $\left(\log \mathrm{P}_{1.4}<22.5\right.$; within the SFdominated regime) is also suggested by the radio luminosity function for that region, and brief colour analysis suggests that such an excess is indeed predominantly associated with a starforming population. The differences between the two filamentary zones is attributed to cosmic variation. The regions are both small $\left(\sim 1^{\circ 2}\right)$, and are significantly separated in the HRS. Further radio observations of filaments are required and the results combined into a larger sample size in order to arrive at a generalised model filamentary population.
\end{abstract}




\section{Contents}

1 Introduction 3

1.1 Filaments and the Large Scale Structure of the Universe . . . . . . . . . . . 3

1.2 Active Galactic Nuclei and Star-forming Galaxies . . . . . . . . . . . . . . 5

1.3 Star-Forming Radio Galaxies in Rich Clusters . . . . . . . . . . . . . . 7

1.4 Hardware . . . . . . . . . . . . . . . . . . . . . . . . . 10

1.4.1 The Australia Telescope Compact Array . . . . . . . . . . . . . . . . 10

1.4.2 Computer Hardware . . . . . . . . . . . . . . . . . . . . . . 11

1.5 Research Aims . . . . . . . . . . . . . . . . . . . . 12

2 Early Results $\quad 15$

2.1 Reduction of Archival Data . . . . . . . . . . . . . . 15

2.2 Analysis of Fornax Beta Field Observational Data . . . . . . . . . . . . 25

2.2 .1 Radio Source Counts . . . . . . . . . . . . . . . 25

2.2 .2 Radio Luminosity Function _. . . . . . . . . . . . . . . . . 27

3 ATCA Observations of Zone 1 and Zone 2 Filaments 35

3.1 Radio Observations and Reduction . . . . . . . . . . . . . . . . 35

3.1 .1 Region Selection . . . . . . . . . . . . . . . 35

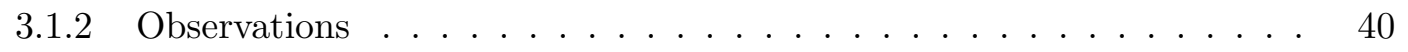

3.1 .3 Reduction Process . . . . . . . . . . . . . . . . 43

3.1 .4 Image Preparation . . . . . . . . . . . . . . . . . . . . 47

3.1.5 Source Detection . . . . . . . . . . . . . . . 50 
4 Analysis of Zone 1 and Zone 2 Filaments 53

4.1 Radio Intensity Properties of Zones 1 and $2 \ldots \ldots \ldots \ldots$

4.1 Source Counts . . . . . . . . . . . . . . . . . . . 53

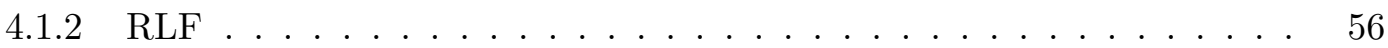

4.1.3 Colour Analysis of Radio-Weak Sources . . . . . . . . . . . . . . . . 61

4.1 .4 Spectral Indices . . . . . . . . . . . . . . . . . . 63

4.2 Polarisation Properties of Zones 1 and $2 \ldots \ldots \ldots \ldots$

4.2.1 Generation of Polarisation Images . . . . . . . . . . . . . 75

4.2.2 Analysis of Polarised Sources in Zones 1 and $2 \ldots \ldots$. . . . . . . 75

5 Discussion $\quad 85$

5.1 Source Counts in Zones 1 and $2 \ldots \ldots \ldots \ldots$. . . . . . . . . 85

5.2 False Detection of Sources with Duchamp . . . . . . . . . . . . 86

5.3 Radio Luminosity Functions . . . . . . . . . . . . . . . . . . . . 87

5.4 Spectral Indices of Filamentary Populations . . . . . . . . . . . . . . . . . 89

5.4 .1 Spectral Indices and CABB . . . . . . . . . . . . . . 89

5.5 Linear Mosaic vs Joint Deconvolution _ . . . . . . . . . . . . . . . . . . . . 91

6 Conclusions and Further Work $\quad 93$ 


\section{Chapter 1}

\section{Introduction}

\subsection{Filaments and the Large Scale Structure of the Universe}

It is widely accepted that a large percentage of matter in the Universe is contained within filaments of galaxies. Aragón-Calvo et al. (2010) propose that filaments represent 39 percent of the mass of the Universe concentrated into only 10 percent of the volume. Bond et al. (1996) estimate a mass as high as 50 percent of that of the Universe, in the same volume. These filaments are connected together to form the web-like large-scale structure of the cosmos (Fig. 1.1). The intersections of filaments are marked by the presence of galaxy clusters, each comprising hundreds of gravitationally-bound galaxies. Over Myr-timescales galaxies are drawn along cosmic filaments toward clusters by gravitational attraction, undergoing evolution as they do so. This follows naturally from the change in environment, which is a major factor in determining physical properties of galaxies such as morphology and star formation rate (Porter et al. 2008).

Filaments of galaxies have been well-studied in a number of observational wavelength regimes. Recent wide-field surveys such as the two-degree Field Galaxy Redshift Survey (2dFGRS, Colless et al. 2001) have probed these structures in the optical band, allowing us to characterise their organisation and geometry. Fadda et al. (2008) used infra-red observations to detect two filamentary structures between the Abell clusters A1763 and A1770. A number of studies have detected X-ray emission from diffuse gas in regions between galaxy clusters, leading to the positive identification of filamentary structure (Kull and Böhringer 1999; Scharf et al. 2000; Durret et al. 2003). Additionally the thermal Sunyaev-Zel'dovich (tSZ) has been used as a detector for filamentary structure that is not redshift dependent (Planck Collaboration et al. 2013). Finally, filaments have been observed indirectly using gravitational lensing techniques (Dietrich et al. 2005). In many cases filamentary detections in one regime are later confirmed by observations in other bands. Planck Collaboration et al. (2013) is a good example of this, in which skeptical detection of a filament via X-ray observation was later confirmed via detection of tSZ emission.

Such studies have revealed filaments to contain an excess of low-powered, star-forming galaxies, and that the rate of star formation generally decreases as we move closer to cluster centres - star forming processes becoming quenched by the high densities typical of these regions. 


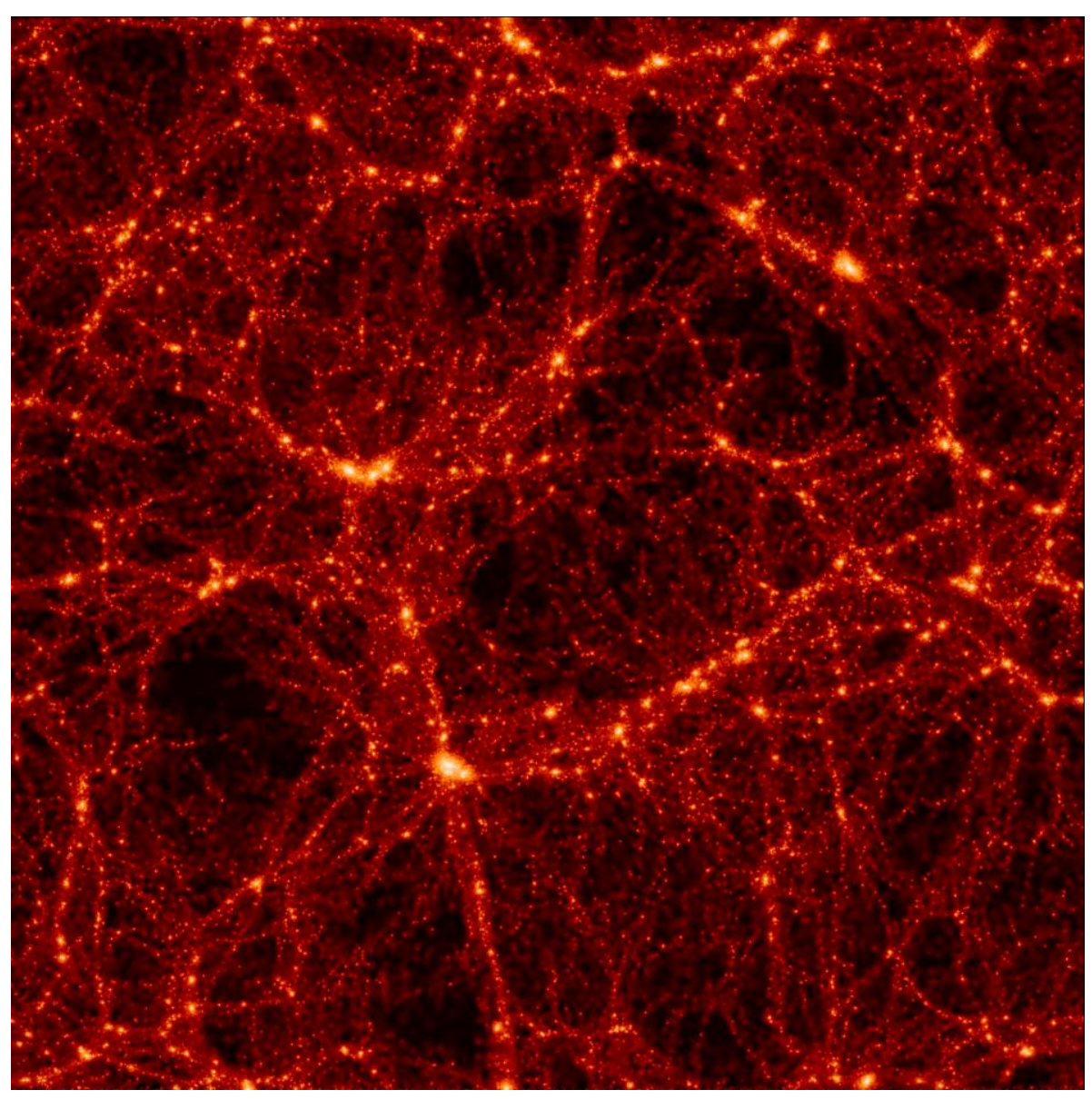

Figure 1.1: Mass distribution plot showing the results of a simulation of sources in the local universe, performed by the Virgo Consortium. Simulation modelled $256^{3}$ particles, and used a version of the AP3M N-body code on two large Cray T3D parallel supercomputers at the Edinburgh Parallel Computing Center and the Computing Centre of the Max Planck Society in Garching. Image shown is Lambda CDM, with $\lambda=0.7$ and $z=0.0$. Evident in the image is the large scale structure of the universe, with matter preferentially coalescing into filaments, creating voids of relative low density between them. Intersections of filaments are marked by galaxy clusters; dense, dynamic regions which appear as bright hotspots in the above map (Jenkins et al. 1998). 
However, in a survey sample of 52 filaments Porter et al. (2008) note the presence of an unexpected peak in the star formation rate at a distance of $2-3 \mathrm{~h}_{70}^{-1} \mathrm{Mpc}$ from the centres of clusters into which galaxies are falling. They attribute this to interactions with other infalling galaxies resulting in a sudden enhancement in star formation rate, before this is inevitably quenched by the cluster.

Despite a growing body of work that deals with cosmic filaments in other wavelengths, no study has yet probed these structures at radio wavelengths. The primary reason for this was alluded to above. Filaments predominantly contain low-powered sources. Until recently instruments with sufficient sensitivity to observe filamentary radio sources have not been available. However, every successive generation of radio instruments offers us significant advances in observational power from that which came before. While the largest leap forward is expected to come with the eventual completion of the Square Kilometre Array (SKA) telescope, notable advances are being made regularly with other, smaller facilities. The CABB-upgraded ATCA (Compact Array Broadband Back-end, Australia Telescope Compact Array; Wilson et al. 2011) is one such radio telescope. This instrument, as well as other projects like ASKAP (The Australian SKA Pathfinder; Johnston et al. 2008; Norris et al. 2011), allow for previously unachievable levels of sensitivity, making radio a cost-effective and increasingly powerful tool for observing and surveying low-powered sources.

\subsection{Active Galactic Nuclei and Star-forming Galaxies}

Radio emission of extragalactic origin can be divided into three types, based on scale. The largest of these is diffuse radio emission. This can be subdivided into relics and halos, Mpcscaled emission found in clusters, and not associated with particular galaxies. The dynamic state of clusters affects the properties of diffuse emission, or whether it is present at all. Because both kinds of diffuse emission are cluster-based phenomena not found away from rich centres, they will take no further part in this study.

The second-largest emission type is synchrotron emission by active galactic nuclei (AGNs). In this scenario matter infalling along accretion disks in elliptical galaxies results in the expulsion of highly relativistic jets of plasma, on an axis perpendicular to the disk. These jets typically extend much further than the host galaxy; radio tail lengths exceeding $\sim 1$ $\mathrm{Mpc}$ are not uncommon. A useful property of AGNs is that they are good indicators of environment, particularly in clusters. In a relaxed environment radio tails will extend in approximately straight lines from the poles of the host galaxy (possibly with some visible oscillation if the host is rotating). However interaction with the dense intracluster medium (ICM) will constrict radio jets. Relative motion between host and ICM will result in the two jets being swept backward, in extreme cases into a single observed tail (Miley et al. 1972). These head-tail (HT) galaxies can be described by the degree of sweeping; wide-angle-tail (WAT) or narrow-angle-tail (NAT) radio galaxies (Rudnick and Owen 1976). AGNs are more commonly found in rich clusters. A classical example, Cygnus A is shown in Fig. 1.2 (Carilli and Barthel 1996). In this galaxy we clearly see the two radio jets extending from the central host. At the termini of the jets we see hotspots, localised areas of increased radio flux. From the orientation of the jets, at $180^{\circ}$ to one another, we can state that this galaxy is not moving relative to the ICM in the plane of the sky (velocity along the line of sight must be determined 


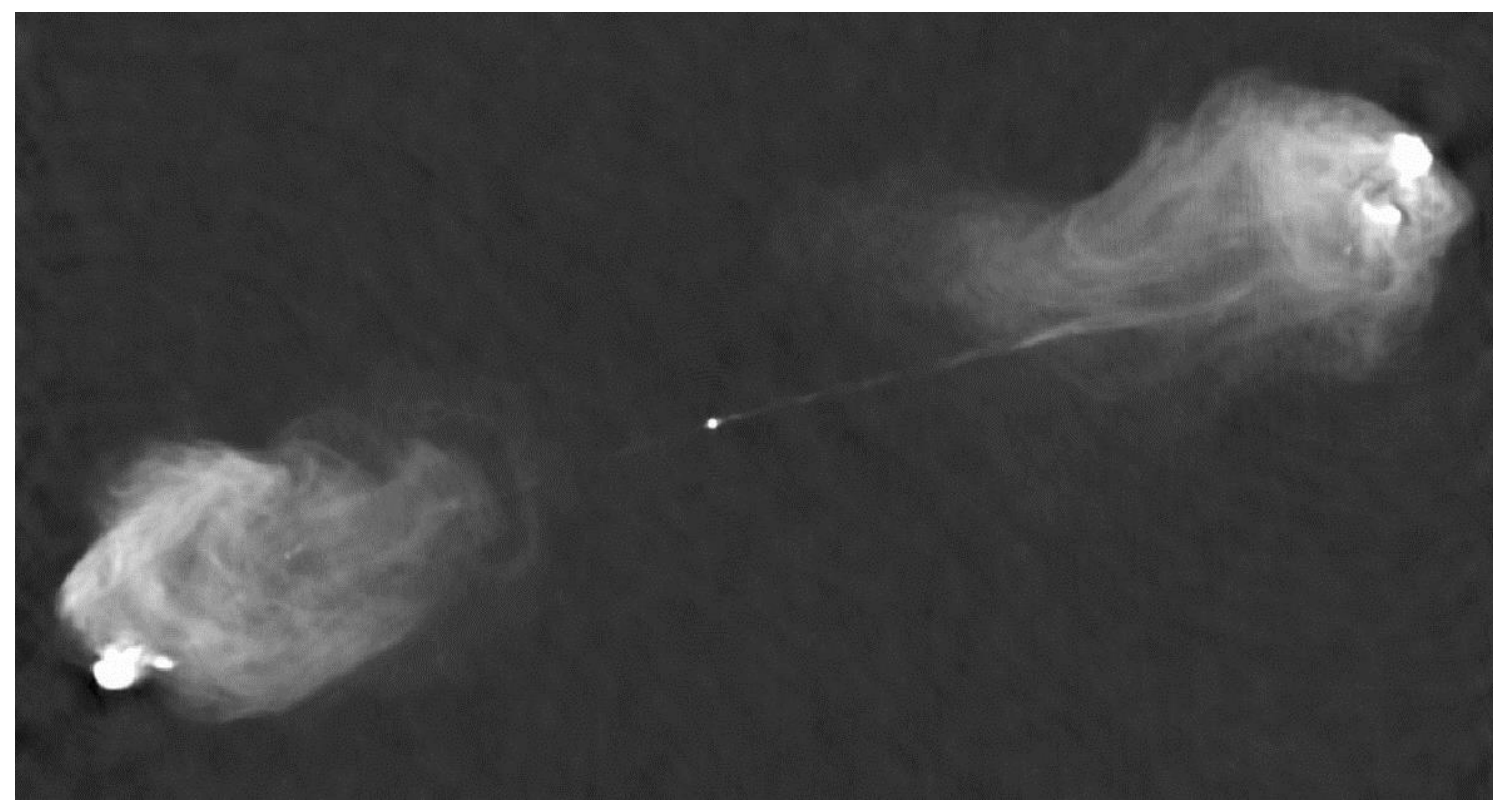

Figure 1.2: Cygnus A, a well known example of an active galactic nucleus (AGN). The pair of radio jets extend from either pole of an elliptical host galaxy (centre) as matter is drawn inwards along its accretion disk (Carilli and Barthel 1996).

by other means, e.g. redshift).

The smallest-scale mode of radio emission detectable from extragalactic sources is from star formation in spiral galaxies. In spiral galaxies like the Milky Way, an excess of molecular gas and dust provides the fuel for the creation of new stars in diffuse nebulae (known colloquially as stellar nurseries). The stars formed in these regions are typically hot. Stellar temperatures of $\mathrm{T} \sim 10^{4} \mathrm{~K}$ result in thermal radiation peaking in the blue part of the optical spectrum. These young stars also emit in the radio band via synchrotron emission, similar to that seen in AGNs but on a much smaller scale (Condon 1992). While these radio stars are able to be detected within the Milky Way on an individual basis (Taylor and Seaquist 1985), for extragalactic objects this is unfeasible. Rather we see the integrated signature of starforming across a galaxy as a whole. Because the radio emission here is associated with objects within a galaxy, rather than the galaxy itself (as for AGNs), starforming galaxies at comparable redshifts are rather less spectacular. Radio jets from AGNs can continue well beyond the limits of an observed optical host but emission in star forming (SF) galaxies is usually limited to within, or slightly beyond the spiral itself. For nearer examples with suitable orientations, emission from star formation can be seen to follow the arms of the host spiral. More commonly distant SF galaxies are observed by detection of point sources, and confirmed by spectral analysis. An example of a powerful SF galaxy is shown in Fig. 1.3, showing the typical scale of observed radio emission.

In studying the local radio luminosity function (RLF) of 6667 sources drawn from NVSS (NRAO VLA Sky Survey; radio) and 6dFGS (6-degree-Field Galaxy Survey; optical), Mauch and Sadler (2007) found that the total RLF could be described by superposition of RLFs for AGN- and SF- populations, allowing them to divide the radio population into two regimes 


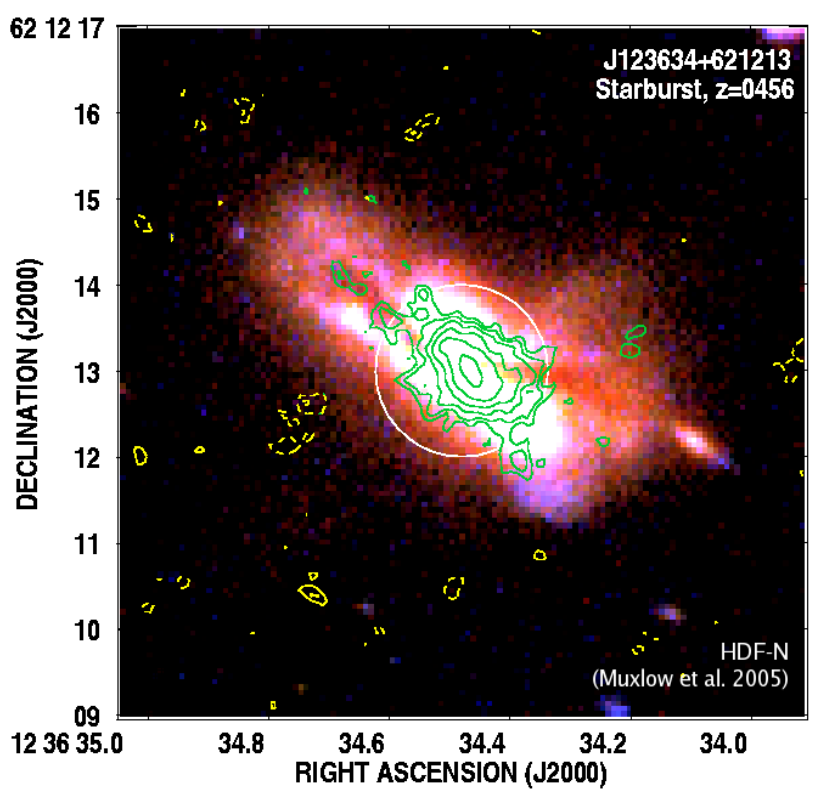

Figure 1.3: Star formation in spiral galaxy J123634+621213, detected with MERLIN+VLA at $1.4 \mathrm{GHz}$ (green contours). Typically for a SF galaxy, the radio emission here is limited to the approximate size of the host galaxy, and shows some of the spiral structure observable in the optical (HST ACS) image (Muxlow et al. 2006).

by radio power (see Fig. 1.4). For radio powers $\log \mathrm{P} \geq 23$ the population is dominated by radio-loud AGNs. In the low power regime, $\log \mathrm{P} \leq 22.5$ radio-quiet AGN make up the majority of the population. At some radio power between these two ranges, there will be a crossover point where the population consists of an equal mix of the two species. According to Miller and Owen (2002) a division can be made in a cluster population at $\mathrm{P}_{1.4}=10^{22.8}$ $\mathrm{WHz}^{-1}$, below which starforming galaxies dominate. At $\mathrm{P}_{1.4} \geq 10^{22.8}$ the population will be dominated by AGNs. Other authors (e.g. Morrison and Owen 2003; Condon et al. 2002; Haines et al. 2011) arrive at slightly different values for this crossover, but typically these fall within $\log \mathrm{P}_{1.4}=22.8 \pm 0.2$.

\subsection{Star-Forming Radio Galaxies in Rich Clusters}

Although at the time of writing no studies have probed filamentary radio populations in the low-powered, predominantly star-forming regime (defined below), a number of papers deal with the star forming properties of galaxies in and around clusters at this frequency (e.g. Giacintucci et al. 2004; Venturi et al. 2000; Miller and Owen 2003). Radio emission is a strong indicator of the activity (AGN, star formation) in galaxies (Condon 1992). While this is true everywhere it is more relevant in clusters where radio sources are as a rule more powerful - and more easily observed - than those in the field. Typically such studies deal with whole cluster radio populations, noting the relative abundance of starforming galaxies and active galactic nuclei (AGNs) via analysis of radio source counts and/or the radio luminosity 


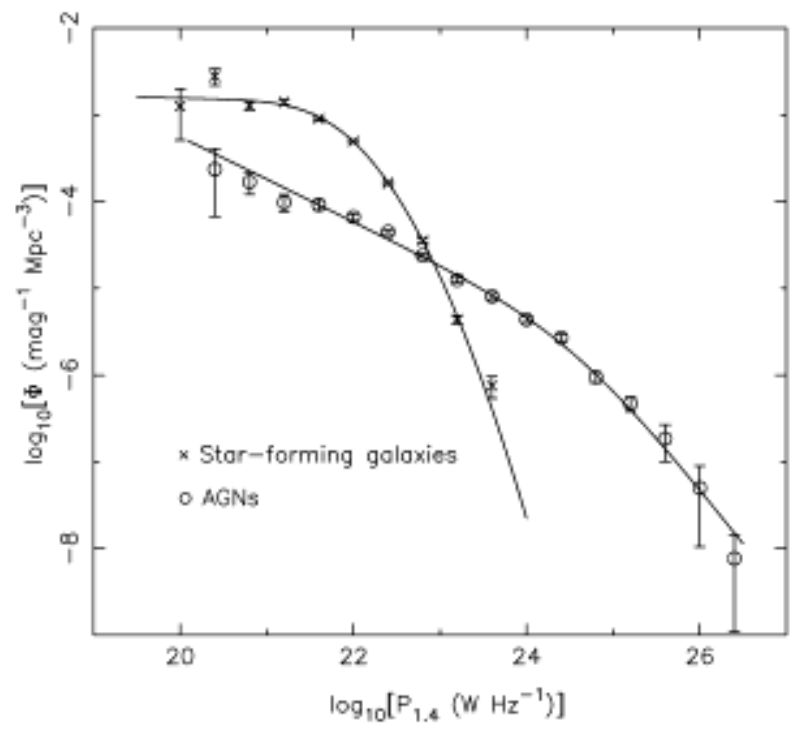

Figure 1.4: Radio luminosity function for 6667 radio/optical sources drawn from NVSS6dFGS sample. The overall RLF can be divided into RLFs for the SF population - which dominates in the low power regime, and the AGN population - which dominates at higher radio powers (Mauch and Sadler 2007).

function (RLF) of the population. Significant deviation from canonical studies (generally Prandoni et al. (2001) and Ledlow and Owen (1996) for source counts and RLF, respectively) are noted, and considered in terms of the morphology and dynamic state of the host cluster. A (non-exhaustive) selection of studies are summarised below.

In this fashion Owen et al. (1999) compared the results of 20cm VLA observations of rich clusters A2125 and A2645, both of which had been considered previously by Butcher and Oemler (1984). Despite the two clusters being almost identical in redshift and richness, their radio populations were found to be markedly different. While A2645 exhibits relative and overall abundances in keeping with canonical studies, A2125 contains a significant excess of galaxies with $\log \mathrm{P}_{1.4} \leq 23$, as well as an excess in overall source counts. The authors concluded that the discrepancy between the two clusters was due to the different dynamic states; A2645 is a relaxed cluster, while A2125 shows evidence of a current cluster/cluster merger taking place. The active starforming galaxies are noted to lie on the periphery of apparently merging clumps, suggesting that this activity has something to do with the unusual properties of the cluster.

Similarly Miller and Owen (2003) considered the case of the merger system A2255, comparing it to a number of other rich Abell clusters (details in Miller and Owen 2002). They noted an excess of both AGN and starforming galaxies. Optical emission line analysis revealed that the excess of optically dim sources was due almost exclusively to starforming in these galaxies. These galaxies showed evidence of current or recent starforming, pointing to a recent increase in activity probably accounted for by the merging environment. Spatial analysis of starforming galaxies revealed that the majority are aligned perpendicular to the proposed 
merger axis. Additional correlations between the radio galaxy fraction (determined from the RLF) and (i) cluster richness, and (ii) cluster compactness, were looked for but invalidated.

The studies of Venturi et al. (2000) and Giacintucci et al. (2004) have focused on the A3558 complex, a post-merger system at the heart of the Shapley Supercluster (Bardelli et al. 1998). Venturi et al. found that the radio source count profile for the system was comparable to the background figures, implying that an increased optical density does not necessarily correspond with a similarly increased radio density. For A3558 the RLF showed a deficit for radio powers $\log \mathrm{P} \geq 22.5$ relative to that of Ledlow and Owen (1996). Giacintucci et al. derived an RLF for more sources, covering a greater area in the system, leading them to conclude that the the earlier-identified deficit was associated with A3558 itself. Despite noting a large percentage $(\sim 50 \%)$ of blue galaxies, they found only weak evidence of an increased starforming population in the system, compared to non-merging environments. Furthermore, Venturi et al. concluded that at best merging has no influence on the probability of a galaxy becoming a radio source nor on its emissivity if it does, and at worst that merging anticorrelates with radio emission.

Finally, Johnston-Hollitt et al. (2008) analysed 1.4 and $2.5 \mathrm{GHz}$ observations of A3158 in the Horologium-Reticulum Supercluster. They saw an excess of source counts for $\log \mathrm{P}_{1.4} \leq 22.3$ galaxies, as well as a significant blue excess, consistent with a boosted starforming population. The positions of the starforming sources indicate merging in the cluster as the means for the ignition of star formation. The RLF for A3158 was lower than that of Ledlow and Owen (1996), but similar to RLFs of clusters in the Shapley Supercluster (e.g. Venturi et al. 2000). Also, as in Miller and Owen (2003), the starforming galaxies were found to lie along the merger axis within the cluster, suggesting some connection between merging environment and galaxy type.

From these and other studies a confused picture begins to emerge as we attempt to probe the relationship between cluster environment and radio activity in the form of AGNs and star formation. This has been frequently noted. On the one hand a galaxy falling into a dense cluster would experience ram pressure as a result of interaction with the intracluster medium (ICM) (Gunn and Gott 1972) and this may trigger star formation as molecular clouds are compressed (Dressler and Gunn 1983). Conversely it is thought that while denser clouds may be compressed, in many cases ram pressure could strip the neutral gas from a galaxy completely, quenching star formation and precluding its resumption at a later time (Miller and Owen 2003, and references therein). Quilis et al. (2000) estimate that such stripping could take as little as $\sim 10^{8}$ years. Simulations of cluster dynamics have led Roettiger et al. (1996) and Caldwell and Rose (1997) to suggest compromised theories, which include initial compression followed by stripping due to the formation of a protective bowshock about the infalling galaxy.

The question endures because of a lack of data. Although the aforementioned studies analyse several rich clusters, their findings often disagree. Furthermore, they generally only probe radio sources at the upper limit of the starforming regime. As a result, it is unclear what we should expect to see as we work our way along filaments in the radio. Most sources cite some connection between a merging environment and starformation. If merging induces star formation, then filaments should show a deficit of such sources. If a turbulent environment quenches star formation then there may be an observable excess of low powered sources consistent with a starforming population. At the least, deep radio observations into filamentary 
regions should detect the marked increase in star formation noted in the optical observations of Porter et al. (2008) at the $2-3 \mathrm{~h}_{70}^{-1} \mathrm{Mpc}$ mark, if this is both true and universal.

\subsection{Hardware}

Probing the low-power, SF-dominated regime is not outside the capabilities of currentgeneration radio telescopes - for example VLA (Very Large Array) and ATCA (Australia Telescope Compact Array) - observing over periods of a few hours. However continuum observations to this depth yield a massive data output that must be manipulated and reduced before any analysis is possible. For this reason computing represents a key component in the overall hardware integral to modern radio astronomy.

\subsubsection{The Australia Telescope Compact Array}

The Australia Telescope Compact Array is a radio instrument consisting of six $22 \mathrm{~m}$ dishes, located outside of Narrabri, NSW. Five of these antennas are mounted on a $3 \mathrm{~km}$ east-west track, with the sixth in a fixed position to the west, allowing for a total baseline of $6 \mathrm{~km}$. A six-element telescope provides 15 unique baseline lengths, which each sample a different elliptical arc in 2D Fourier space. The east-west configuration allows for complete angular sampling of the Fourier domain (called the $U V$ plane in radio astronomy) over a 12 hour period, in a process known as Earth-rotation aperture synthesis (for which Martin Ryle and Antony Hewish were jointly awarded the 1974 Nobel Prize for Physics). The eccentricity of elliptical arcs depends on the declination of the source, meaning that optimum sampling will be achieved for sources in the deep south, while equatorial sources will provide something of a challenge for effective observing. Using several baseline configurations additionally fills out the UV plane by providing a greater number of unique baseline lengths. If necessary data from the ATCA can be combined with single-dish observations (e.g. from Parkes) to eliminate the issue whereby a lack of a zero-length baseline results in a gap in the UV-coverage at the origin. However, this is only an issue when observing sources on scales greater than $\sim 20$ arcminutes so, in general, can be ignored.

The CABB upgrade for the ATCA was completed in 2009, offering a significant improvement in the overall effectiveness of the instrument. In this upgrade the observing band of the ATCA was increased from $128 \mathrm{MHz}$ to $2 \mathrm{GHz}$, and maximum continuum-mode channels boosted from 32 to 2048. This, in combination with higher level data sampling has improved the continuum sensitivity of the ATCA by at least a factor of four as well as providing a greatly enhanced spectral line performance, particularly at the higher observing frequencies ${ }^{1}$.

The expected RMS flux sensitivity $\mathrm{S}$ that can be probed by a radio telescope is described by the relation

$$
S \propto \frac{1}{\sqrt{\nu \cdot t}}
$$

Where $\nu$ is the observing frequency and $t$ is the integration time. Thus for a given system in which all other factors remain the same, we can relate the times $t_{1}$ and $t_{2}$ taken to probe to

\footnotetext{
${ }^{1}$ http://www.narrabri.atnf.csiro.au/observing/CABB.html
} 
two separate sensitivity levels $\mathrm{S}_{1}$ and $\mathrm{S}_{2}$ by

$$
S_{1}^{2} t_{1}=S_{2}^{2} t_{2}
$$

This allows us to use the quoted sensitivity of an instrument (flux sensitivity $\mathrm{S}_{\text {sys }}$ for a given integration time $\mathrm{t}_{s y s}$ ) to calculate the required integration time $\mathrm{t}_{\text {obs }}$ to probe to a desired level $\mathrm{S}_{\text {obs }}$. Rearranging:

$$
t_{\text {obs }}=\left(\frac{S_{\text {sys }}}{S_{\text {obs }}}\right)^{2} \cdot t_{\text {sys }}
$$

In the $16 \mathrm{~cm}$ wavelength band the sensitivity of CABB is quoted at $0.03 \mathrm{mJy} /$ beam for an integration time of 10 minutes $^{2}$ meaning that probing the radio sky at the $\mu J y$-level in a reasonable time, is now a realistic possibility with the ATCA.

Now, according to Condon (1992) the starforming rate of a galaxy is given by

$$
S F R_{\nu}\left(M \geq 5 M_{\odot}\right)=\frac{P_{\nu}}{4.0 \times 10^{21} W H z^{-1}} M_{\odot} y r^{-1}
$$

Different studies (e.g. Hopkins et al. 2003; Rieke et al. 2009; Haines et al. 2011) have arrived at slightly different values for the denominator, but these variations are not large enough to seriously affect our calculations. The value $\mathrm{P}$ is the radio power of the galaxy:

$$
P_{\nu}=\frac{4 \pi \cdot D_{L}^{2} \cdot S_{\nu}}{(1+z)^{(1-\alpha)}}
$$

$D_{L}$ is the luminosity distance of the galaxy (in $\mathrm{m}$ ), $\mathrm{S}_{\nu}$ is its observed radio flux (in $\mathrm{W} / \mathrm{m}^{2} / \mathrm{Hz}$ ), $\mathrm{z}$ is its (dimensionless) cosmological redshift and $\alpha$ the spectral index, which is defined elsewhere in this text. All conversions between radio flux and radio power in this study assume sources have a redshift equal to the average redshift of the HRS, $\mathrm{z}=0.0623$.

Combining these relations with Eqns 1.3 and 1.5 allows us to relate the integration time necessary to probe a given starforming rate, if we know the observing frequency, instrumental sensitivity and distance and spectral index properties of the source galaxies(s).

\subsubsection{Computer Hardware}

The initial stages of this project, including some CABB data reduction, were completed using a Linux-based operating system on a consumer-level computer. This resulted in limits on speed, as well as the size of data that could be manipulated in reduction. From September 2012 the majority of data reduction and manipulation was able to be transferred over to SPOCK, one of a pair of supercomputers hosted by Victoria University of Wellington, and similar in specifications to the nodes used at the MWA (Murchison Widefield Array) facility in Australia (Tingay et al. 2012). The pair is contained in a RADON Duo G2650 IBQ Twin2U (a Super Micro SuperServer 6026TT-GIBQRF with a X8DTT-HIBQF+ motherboard) manufactured by Xenon Systems Pty Ltd., of Australia. According to the initial hardware specifications, each machine contains:

\footnotetext{
${ }^{2}$ ATCA User Guide, available online http://www.narrabri.atnf.csiro.au/observing/users_guide/
} 
- 2x Intel Xeon X5650 Six-Core processors - 2.66 GHz 6.4 GT/s

- 96 MB DDR3-1333 ECC Registered DDR Module RAM

- 6x WD 2TB RE4 SATA II, 7200 RPM 64 MB cache HDD

In addition, the nodes both contained Tesla 2070 GPGPU cards. However, for the scale and type of computing used in this study the use of these was not necessary.

\subsection{Research Aims}

The major aim of this study was to perform the first benchmarking study of radio sources within cosmic filaments. Several of the studies discussed in section 1.3 revealed an excess of low powered sources in the radio populations in and around clusters, which is consistent with the trends identified in other wavelengths. However, these analyses have generally been relatively shallow, only probing sources with $\log \mathrm{P}_{1.4}>21.8$, which is only the uppermost extent of the star forming regime. Another notable point is that these previous studies have only explored filamentary populations as a by-product of cluster observation. This means that the observations are spatially limited to filamentary termini, and can offer only limited insight into the overall nature of filaments.

The particular filaments selected for study in this thesis are located within the HorologiumReticulum Supercluster (HRS). This massive structure, centred at approximately RA 03h13m and Dec $-50^{\circ} 02^{\prime \prime}$, covers an area in the sky of more than $12^{\circ} \times 12^{\circ}$ and contains upward of 30 Abell clusters, as well as numerous smaller galaxy groups (Fleenor et al. 2005). The reasons for choosing the HRS as our focus were several; first, the HRS represents the second largest mass concentration in the local Universe; second, much of the filamentary structure within the supercluster has already been determined by prior optical spectroscopy; third, the HRS offers a microcosm of the greater Universe. The range of environments in the supercluster provide a useful laboratory where comparisons can be made whilst retaining controls on, for example, age and redshift of subpopulations; finally we have pragmatic concerns. The position of the HRS in the southern sky makes it readily observable by the ATCA in NSW, Australia. Several clusters within the HRS have been studied in the past by the radio astronomy group at Victoria University of Wellington, including clusters that flank known filaments (see JohnstonHollitt et al. 2008; Mao et al. 2009; Pratley et al. 2013, for examples). This affords familiarity with the region, as well as observational data that may be more directly useful.

Following the treatment in the aforementioned cluster studies, analysis of filamentary populations would be based primarily around the generation of radio source count profiles and RLFs as the two main indicators of a starforming excess.

In total the populations of four separate regions were analysed. For two of these, Filament 17 (selected from a number of potential filaments with publicly available ATCA data. The selection process and naming convention is described in Section 2.1) and Fornax Beta Field ${ }^{3}$,

\footnotetext{
${ }^{3}$ The Fornax Beta Field survey data were observed as part of ASKAP commissioning. The PI for this project was Feain. Data used with permission.
} 
studies made use of archival data from previous observations. This process is covered in Chapter 2. The latter two regions, labelled Zone 1 and Zone 2, and defined in Section 3.1.1, relied on deliberate observations performed at three different occasions in 2012/2013 using the ATCA. The observations themselves are detailed in Chapter 3, with analysis of the resulting data following in Chapter 4. Findings are discussed and complications noted in Chapter 5, and conclusions arrived at in Chapter 6.

Throughout, we have assumed a standard set of cosmological parameters with $H_{0}=73$ $\mathrm{kms}^{-1} \mathrm{Mpc}^{-1}, \Omega_{m}=0.27$ and $\Omega_{\Lambda}=0.73$. 


\section{Chapter 2}

\section{Early Results}

An important initial step for this project was to identify likely regions within the HRS where we might expect to see filamentary structure. The online archive for ATCA data ${ }^{1}$ was searched for any past observations that might provide some insight into the nature of filamentary radio sources in the supercluster. Unlike the clusters themselves, filaments are rarely identified and catalogued as discrete objects. As a result knowledge of the locations of radio filaments in the HRS is poor. However we can make certain assumptions. According to the hierarchical model of the Universe smaller structures are drawn together by mutual gravitational attraction to form larger structures. Matter accretes along preferred filaments toward gravitational wells, where clusters are found. It follows that at some time there should have been some degree of filamentary connection between most pairs of near-neighbours of clusters. Over cosmic timescales these filaments may not endure. Severely extended strands would be particularly susceptible to weakening as their substance is effectively stretched toward the cluster centres at either end.

At the other extreme, very close cluster pairs would not provide a sufficiently long interim region to see proper filamentary structure. This may result in a lack of strong differentiation between radio sources in-cluster, and those belonging to the filament itself. Taking these two points into account, the most promising regions for the study of filamentary sources would occupy a middle-ground; cluster pairs near enough to exhibit a relatively robust connection, yet far enough apart that filament and cluster sources may be fairly distinguished from one another.

\subsection{Reduction of Archival Data}

Beginning with a map of the HRS (see Fig. 2.1) 20 notional filament zones were identified, running between cluster pairs. These zones followed the reverse-C shape of the HRS, extending from A3301 in the north-east, to A3312 in the south-east (though not in order). The two western clusters A3004 and A3009 were deemed to be too far removed from the bulk of the supercluster to have any remaining filamentary connection to it. The details of all 20

\footnotetext{
${ }^{1}$ http://atoa.atnf.csiro.au/
} 


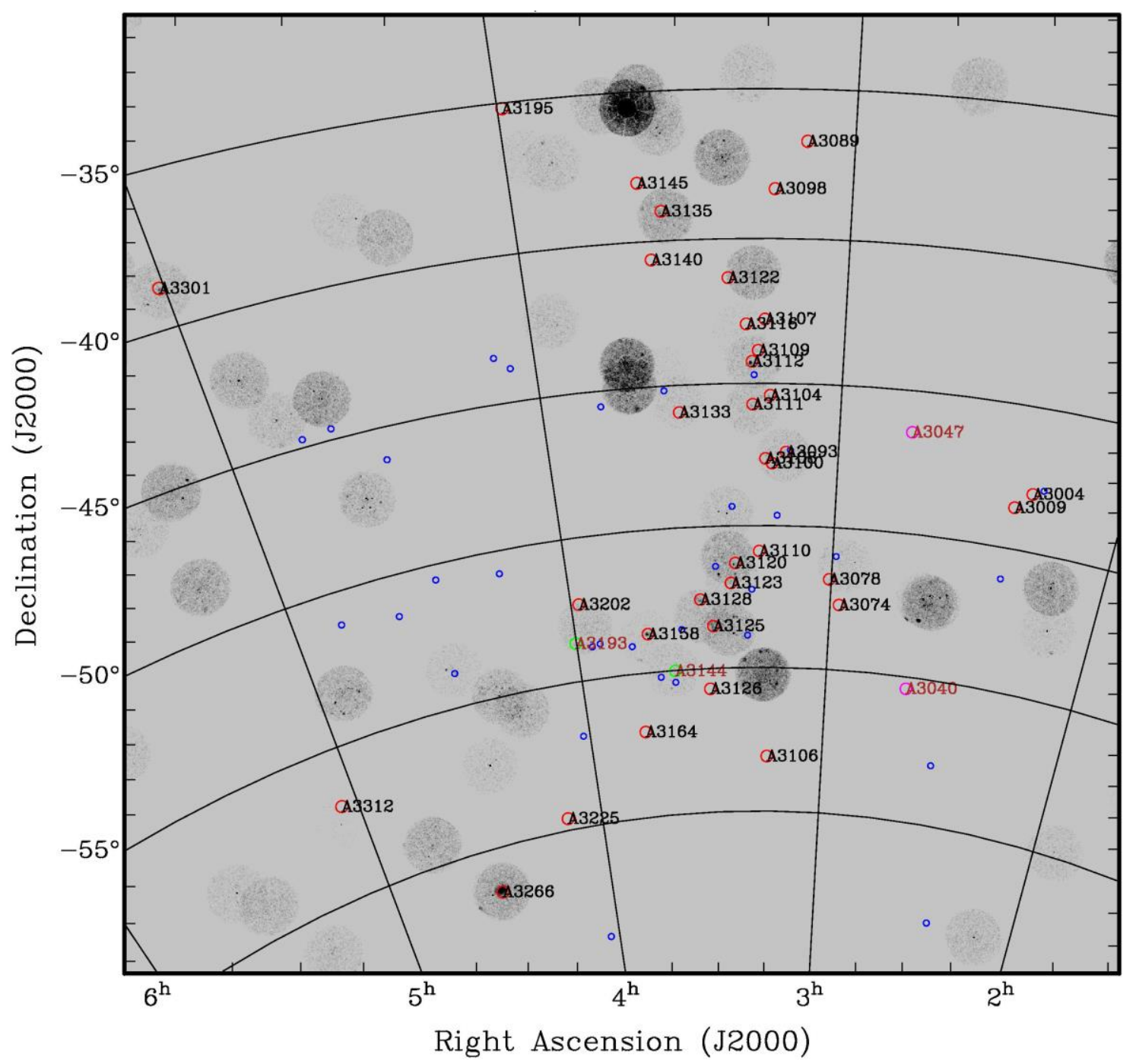

Figure 2.1: Map detailing positions of Abell-cluster members of the HRS (red circles), as taken from Fleenor (2006). Nearby groups are shown in blue, along with two foreground (green circle, brown label) and two background (magenta circle, brown label), clusters. Background is a composite ROSAT PSPC (X-ray) image showing pointed observations to clusters. This is a raw image with uncorrected background levels; it is simply included here for the purpose of outlining the general structure of the HRS. 


\begin{tabular}{ccrlc}
\hline Filament No. & Between Clusters & Centre RA, & Dec (Approx) & Extends (Arcmin) \\
\hline 1 & A3110, A3100 & $03: 16$ & $-49: 13$ & 30 \\
2 & A3110, A3078 & $03: 09$ & $-51: 20$ & 20 \\
3 & A3108, A3111 & $03: 15$ & $-46: 44$ & 20 \\
4 & A3111, A3112 & $03: 17$ & $-44: 58$ & 15 \\
5 & A3107, A3122 & $03: 20$ & $-42: 02$ & 20 \\
6 & A3122, A3135 & $03: 29$ & $-40: 15$ & 30 \\
7 & A3122, A3098 & $03: 18$ & $-39: 47$ & 30 \\
8 & A3145, A3195 & $03: 48$ & $-36: 41$ & 30 \\
9 & A3128, A3123 & $03: 27$ & $-52: 17$ & 10 \\
10 & A3158, A3202 & $03: 52$ & $-53: 38$ & 30 \\
11 & A3125, A3126 & $03: 28$ & $-54: 39$ & 30 \\
12 & A3126, A3164 & $03: 40$ & $-56: 21$ & 30 \\
13 & A3126, A3106 & $03: 22$ & $-56: 56$ & 30 \\
14 & A3202, A3164 & $03: 49$ & $-55: 08$ & 40 \\
15 & A3164, A3225 & $03: 58$ & $-58: 18$ & 30 \\
16 & A3225, A3266 & $04: 21$ & $-60: 38$ & 30 \\
17 & A3266, A3312 & $04: 46$ & $-59: 15$ & 60 \\
18 & A3195, A3301 & $04: 32$ & $-36: 54$ & 120 \\
19 & A3158, A3125/A3128 & $03: 36$ & $-53: 20$ & 60 \\
20 & A3135, A3145 & $03: 36$ & $-38: 30$ & 40 \\
\hline
\end{tabular}

Table 2.1: Definitions of 20 potential filaments within the HRS, extending between neighbouring cluster pairs. Here filamentary designation is made a priori, without any evidence of a source overdensity. Filaments 19 and 20, observations of which made up the bulk of this study, were redesignated Zones 1 and 2 as explained in the text.

notional filaments can be found in Table 2.1. The final two filamentary regions in the HRS, Filaments 19 and 20, were selected as the main regions of interest for this study. Filament 19, connecting A3158 and A3125/A3128 is discussed in Johnston-Hollitt et al. (2008). Filament 20, between A3135 and 3145, is presented in Pratley et al. (2013). The pair, hereafter referred to as Zone 1 and Zone 2 respectively, are also further defined in Section 3.1.1 as appropriate.

For each of these notional filament zones, the approximate centre and radius of the zone were used as parameters in an archival search for deep, continuum radio observations. Although very few of the zones returned no data at all, the only case for which there was both sufficient total observing time and continuum bandwidth, was for Filament 17 (see Fig. 2.2). This zone lies at the south-eastern extreme of the HRS, extending between the clusters A3266 and A3312. In total there were nine projects in the archive with observations covering this region. Details of these observations are presented in Table 2.2. It is important to note here that the archive metadata only provides the total time for an observing project. In most cases the quoted observing time will be divided between several sources observed on the same day.

These observational data were reduced with the MIRIAD suite (Sault et al. 1995), using standard operating procedures for pre-CABB data as outlined in the user manual ${ }^{2}$. Ultimately the need for high resolution images necessitated the abandonment of all data sets without

\footnotetext{
${ }^{2}$ Available online from bima.astro.umd.edu/miriad/userguide_US.pdf
} 


\begin{tabular}{ccccccl}
\hline $\begin{array}{c}\text { Project } \\
\text { Code }\end{array}$ & Date(s) & $\begin{array}{c}\text { Observing } \\
\text { Time (min) }\end{array}$ & $\begin{array}{c}\text { Max } \\
\text { Baseline }\end{array}$ & $\begin{array}{c}\text { Frequency } \\
(\mathrm{MHz})\end{array}$ & $\begin{array}{r}\text { Pointing } \\
\text { RA }\end{array}$ & $\begin{array}{l}\text { Centre } \\
\text { Dec (J2000) }\end{array}$ \\
\hline CX024 & $20 / 01 / 2001$ & 323 & $6 \mathrm{~km}$ & 1384,2496 & $04: 45: 22.1$ & $-59: 13: 56.9$ \\
C934 & $15 / 02 / 2001$ & 102.5 & $375 \mathrm{~m}$ & 1384 & $04: 45: 42.1$ & $-59: 14: 57$ \\
& $15 / 04 / 2001$ & 123.8 & $750 \mathrm{~m}$ & & & \\
C892 & $04 / 08 / 2000$ & 207.5 & $375 \mathrm{~m}$ & 1384 & $04: 45: 42.2$ & $-59: 14: 50.95$ \\
& $05 / 08 / 2000$ & & & & & \\
$\mathrm{C} 530$ & $01 / 12 / 1996$ & 767.5 & $375 \mathrm{~m}$ & 1344 & $04: 40: 08.0$ & $-58: 46: 00$ \\
& $31 / 03 / 1997$ & 683.5 & $1.5 \mathrm{~km}$ & 2496 & & \\
$\mathrm{C} 156$ & $19 / 05 / 1993$ & 2173.5 & $1.5 \mathrm{~km}$ & 1380,2368 & $04: 45: 42.2$ & $-59: 13: 57$ \\
& $20 / 05 / 1993$ & & & & & \\
& $12 / 10 / 1993$ & & & & & \\
$\mathrm{C} 288$ & $31 / 07 / 1993$ & 143 & $750 \mathrm{~m}$ & 1380,2378 & $04: 48: 24.2$ & $-59: 50: 2.43$ \\
& $01 / 08 / 1993$ & & & & & \\
$\mathrm{C} 295$ & $23 / 06 / 1994$ & 51.5 & $6 \mathrm{~km}$ & 1380 & $04: 45: 42.18$ & $-59: 14: 57.11$ \\
& $24 / 06 / 1994$ & & & & & \\
$\mathrm{C} 082$ & $16 / 01 / 1994$ & 624 & $6 \mathrm{~km}$ & 2378 & $04: 45: 47.18$ & $-59: 14: 39.46$ \\
C133 & $05 / 09 / 1993$ & 6 & $6 \mathrm{~km}$ & 1376,2378 & $04: 45: 42.67$ & $-59: 14: 53.42$ \\
\hline
\end{tabular}

Table 2.2: Summary of previous ATCA observations of the region designated Filament 17 at $\sim 1.4$ and $\sim 2.4 \mathrm{GHz}$. All these observations utilised the older, pre-CABB instrument and lack the sensitivity achieved later for filament Zones 1 and 2, using CABB. All were continuum observations, using 33 channels for a bandwidth of $128 \mathrm{MHz}$. Only projects with $6 \mathrm{~km}$ baselines were reduced as part of this project.

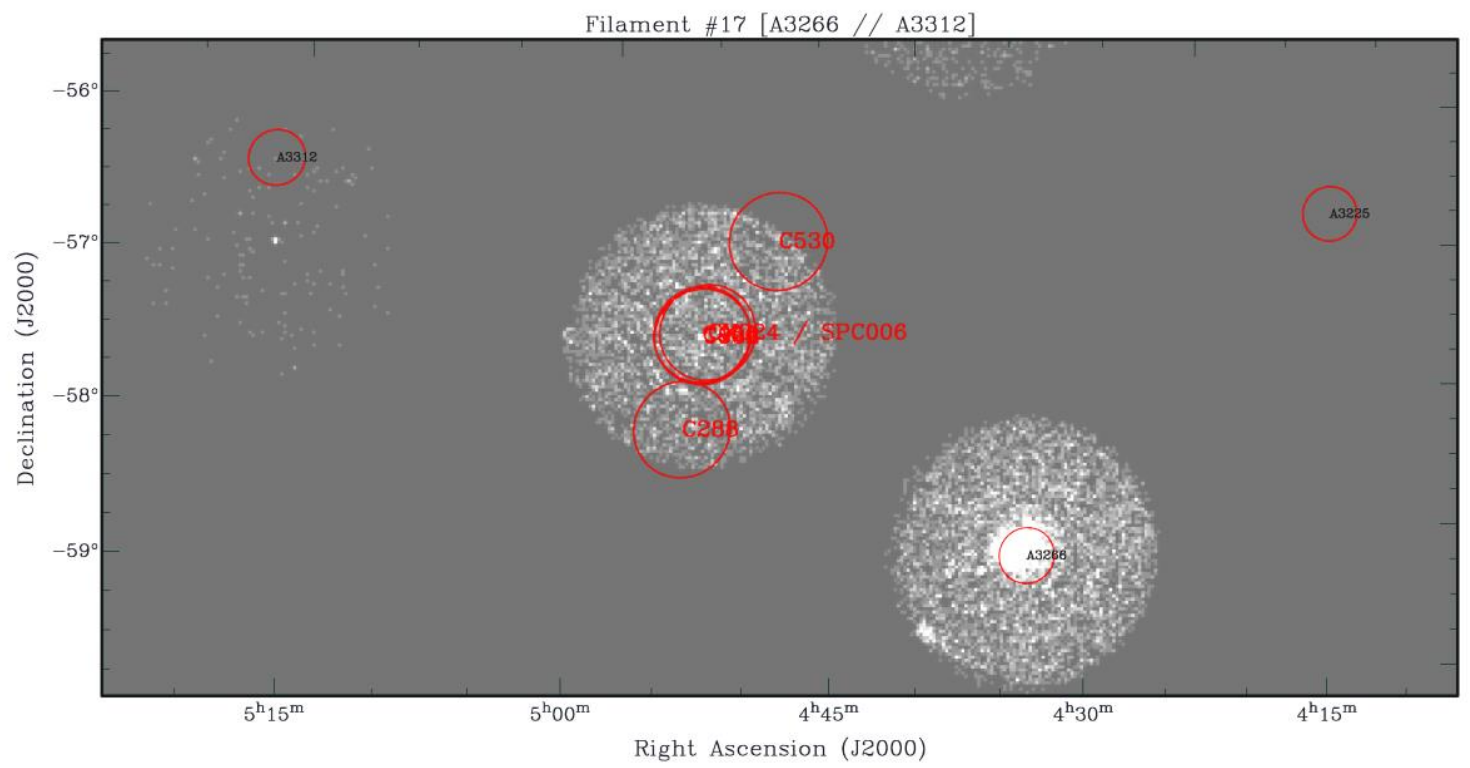

Figure 2.2: Positions of observed fields in Filament 17. With the exception of projects C530 and C288, observations for all projects covered the same position on the sky, roughly equidistant on a straight line between Abell clusters A3312 (upper left) and A3266 (lower right). Background is ROSAT PSPC (X-ray) pointed observations showing locations of Abell clusters A3312 and A3266. 
$6 \mathrm{~km}$ baselines. The remaining data were combined via joint deconvolution into two radio mosaics. The first of these, at $1.4 \mathrm{GHz}$, included data from projects CX024, C133 and C295, and was inverted with a cell size resolution of 2". The second mosaic combined CX024, C082 and $\mathrm{C} 133$ at $2.5 \mathrm{GHz}$, and has a cell size resolution of $1.3 "$. These images were cleaned using the Steer cleaning algorithm, which is suitable for dealing with joint-deconvolved data sets. Final 1.4 and $2.5 \mathrm{GHz}$ radio maps are presented in Figs. 2.3 and 2.4. The $1.4 \mathrm{GHz}$ image had an RMS noise level of $0.5 \mathrm{mJy}$, and a beam of $7.63 " \times 6.25 ", p a=-5.36^{\circ}$. The $2.5 \mathrm{GHz}$ map had an RMS noise level of $0.08 \mathrm{mJy}$, and a beam of $3.54^{\prime \prime} \times 2.79^{\prime \prime}, p a=-17.2^{\circ}$.

From these images a catalogue of radio sources was prepared, consisting of 18 sources this is presented in Table 2.3. The bulk of sources provided flux measurements at $1.4 \mathrm{GHz}$ with corresponding detections at $843 \mathrm{MHz}$ in the SUMSS survey (Mauch et al. 2003), but only six had the data also at $2.5 \mathrm{GHz}$. Integrated flux values at three separate frequencies allow for a pair of spectral indices to be generated for a source, which can be compared on a colour-colour plot. However a population of $n=6$ is too small for this treatment to be useful. Just looking at the spectral index pairs in Table 2.3 shows the full range of cases - steepening, shallowing or roughly flat indices - so certainly more sources would be required before we could expect to see any sort of trend. Postage-stamp images for all sources are shown in Fig. 2.5, with 1.4 $\mathrm{GHz}$ and $2.5 \mathrm{GHz}$ (where applicable) contours overlaid on DSS optical background maps.

What is notable about the population detected in Filament 17 is that of the 18 sources, 14 are extended. These include two HT galaxies (sources 6 and 12), and one double-lobed radio galaxy (source 17). There seems to be little evidence of a significant star forming population in Filament 17 at the depth probed by these observations. A notable exception is source 2, a fine example of a SF galaxy of the type described in Section 1.2. This spiral galaxy is the catalogued source NGC1672 ${ }^{3}$. In the DSS optical background image we can make out the spiral structure of the host galaxy, and we see that radio emission (at both 1.4 and $2.5 \mathrm{GHz}$ ) is confined within this region. The structure of radio emission is not clearly defined, but offers a long axis that appears to be appropriately aligned with the spiral arms of the host. The redshift for this galaxy $(\mathrm{z}=0.0043 \pm 0.0008)$ places it well in the foreground, outside of the HRS. However, it does provide a fine low-z analogue of the kind of system hoped to be detected as part of this study. The postage stamp image in Fig. 2.5 shows only the inner core of NGC1672. A larger image, showing the radio emission in the context of the galaxy as a whole, is presented in Fig. 2.6.

Inspection of the postage stamp image for source 7, initially appears to yield an optical match to the radio detection. This source is located at $\mathrm{RA}=04: 42: 23.8$, Dec $=-59: 01: 06$, and is catalogued as source 2MASX J04422382-5901064. According to that catalogue it has a redshift of $\mathrm{z}=0.0650 \pm 0.00021$, which suggests membership of the HRS. Unfortunately the radio and optical sources are unlikely to be associated to each other, to within the uncertainties of the astrometry here. This seems more likely to be a coincidental superposition than a bona fide radio-optical match.

In Source 5 we see that the emission peaks at 1.4 and $2.5 \mathrm{GHz}$ do not properly coincide, yet the $2.5 \mathrm{GHz}$ contours do fall within the area identified as $1.4 \mathrm{GHz}$ emission. The $2.5 \mathrm{GHz}$

\footnotetext{
${ }^{3}$ This source also marks the pointing centre for the observations reduced in this section, explaining why the field was observed in the first place - falling, as it does, well outside the vicinity of any of the rich clusters in the HRS.
} 


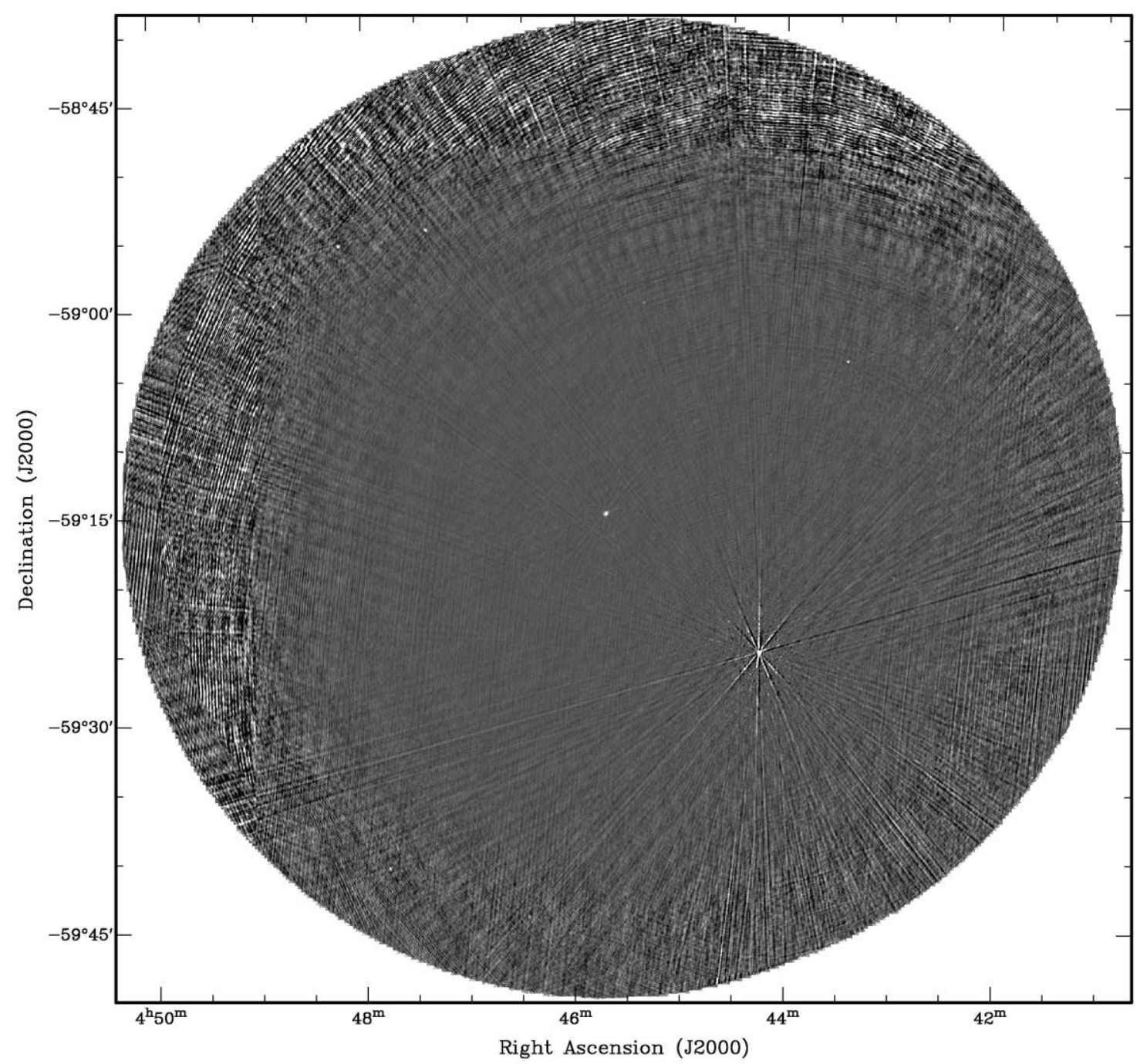

Figure 2.3: $1.4 \mathrm{GHz}$ radio map of Filament 17. The image is a mosaic of observations from projects CX024, C133 and C295. The bright source at the centre of the image marks the position of spiral galaxy NGC1672 (Source 2 in Table 2.3), which was clearly the intended target of these observations. 


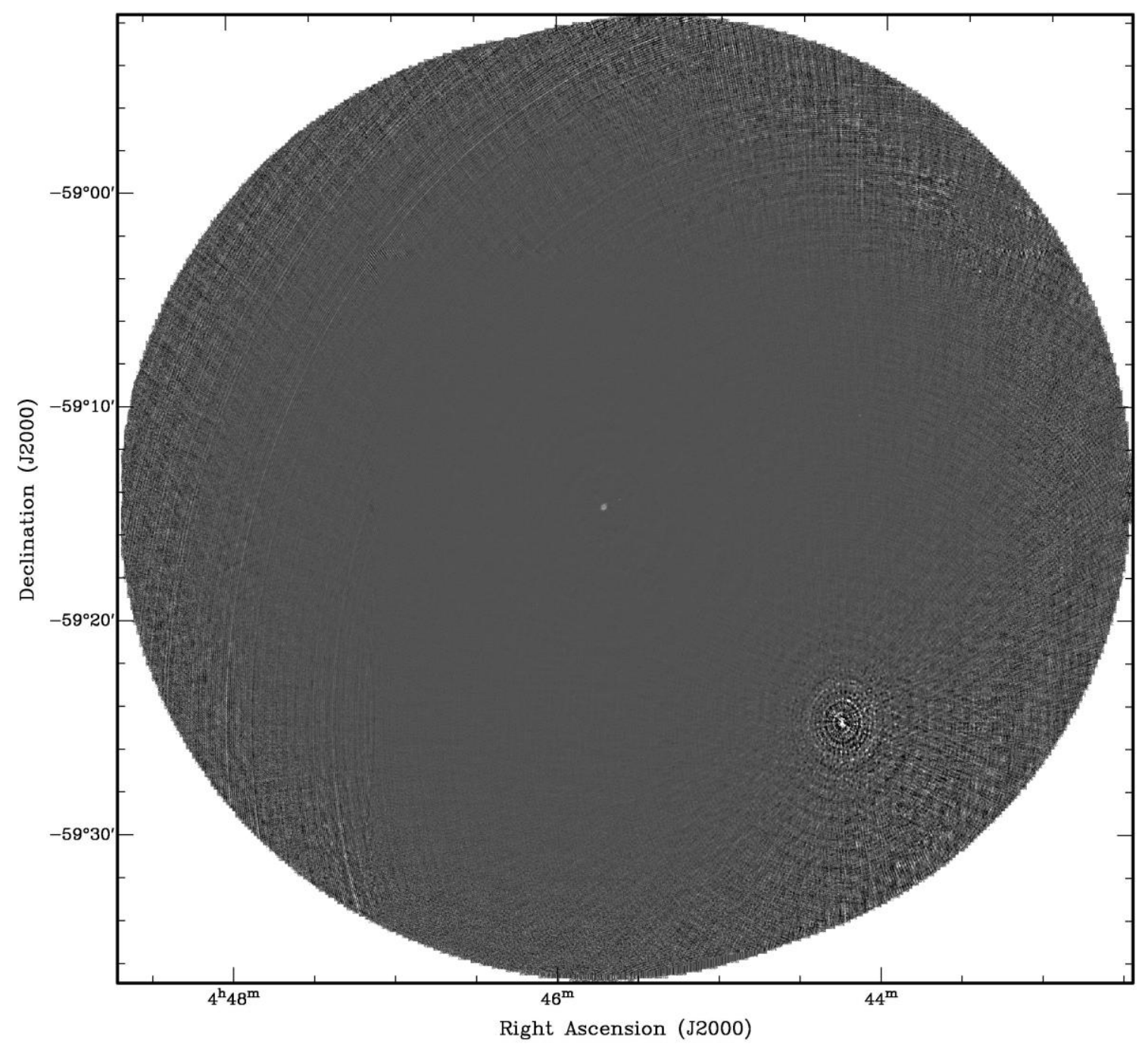

Figure 2.4: $2.5 \mathrm{GHz}$ radio map of Filament 17. The image is a mosaic of observations from projects CX024, C082 and C133. 


\begin{tabular}{|c|c|c|c|c|c|c|c|c|c|}
\hline \multicolumn{2}{|c|}{$\begin{array}{r}\text { Source } \\
\text { No. }\end{array}$} & $\begin{array}{r}\text { Coords } \\
\text { RA }\end{array}$ & $(\mathrm{J} 2000)$ & \multicolumn{2}{|c|}{$\mathrm{S}_{843}(\mathrm{mJy})$} & $\mathrm{S}_{1.4}(\mathrm{mJy})$ & $\mathrm{S}_{2.5}(\mathrm{mJy})$ & $\alpha_{843}^{1.4}$ & $\alpha_{1.4}^{2.5}$ \\
\hline & 1 & $04: 44: 14.0$ & $-59: 24: 54$ & \multicolumn{2}{|c|}{$2080 \pm 33$} & $1190 \pm 4.0$ & $498 \pm 14$ & 1.13 & 1.61 \\
\hline & 2 & \multirow{2}{*}{$\begin{array}{l}04: 40: 41.9 \\
04: 43: 24.6\end{array}$} & \multirow{2}{*}{$\begin{array}{l}-59: 14: 51 \\
-59: 03: 42\end{array}$} & \multicolumn{2}{|c|}{$275 \pm 26$} & $103 \pm 1.9$ & $54.7 \pm 2.8$ & 1.99 & 1.16 \\
\hline & 3 & & & \multicolumn{2}{|c|}{$44.2 \pm 1.3$} & $26.1 \pm 0.42$ & $22.4 \pm 1.1$ & 1.06 & 0.29 \\
\hline & 4 & $04: 45: 20.4$ & $-58: 59: 28$ & \multicolumn{2}{|c|}{$18.1 \pm 1.2$} & $12.0 \pm 0.38$ & $1.85 \pm 0.21$ & 0.83 & 3.45 \\
\hline & 5 & $04: 44: 08.0$ & $-59: 10: 30$ & $4.52=$ & \pm 1.5 & $2.50 \pm 0.31$ & $3.41 \pm 0.22$ & 1.19 & -0.57 \\
\hline & 6 & $04: 42: 57.7$ & $-59: 19: 25$ & $4.27 \pm$ & $=0.36$ & $5.87 \pm 0.91$ & & -0.64 & \\
\hline & 7 & $04: 42: 23.2$ & $-59: 01: 07$ & $29.4=$ & \pm 1.6 & $15.2 \pm 4.1$ & & 1.33 & \\
\hline & 8 & $04: 47: 23.9$ & $-58: 54: 08$ & $117=$ & $=1.9$ & $54.9 \pm 1.7$ & & 1.53 & \\
\hline & 9 & $04: 48: 13.2$ & $-58: 55: 17$ & $161=$ & $=3.9$ & $53.2 \pm 4.3$ & & 2.23 & \\
\hline & 10 & $04: 47: 46.2$ & $-59: 40: 33$ & $73.8=$ & \pm 3.3 & $30.5 \pm 4.0$ & & 1.78 & \\
\hline & 11 & $04: 46: 26.1$ & $-59: 44: 04$ & $78.7=$ & \pm 5.1 & $14.3 \pm 4.0$ & & 3.44 & \\
\hline & 12 & 04:48:50.1 & $-58: 59: 36$ & $80.9 \pm$ & $=0.60$ & $36.0 \pm 5.2$ & & 1.64 & \\
\hline & 13 & $04: 44: 22.0$ & $-58: 58: 21$ & $4.93 \pm$ & $=0.44$ & $1.98 \pm 0.1$ & & 1.84 & \\
\hline & 14 & $04: 42: 24.6$ & $-59: 28: 11$ & $50.3=$ & \pm 3.4 & $16.5 \pm 0.87$ & & 2.25 & \\
\hline & 15 & 04:42:43.7 & $-59: 32: 39$ & 55.4 & \pm 3.2 & $20.5 \pm 1.8$ & & 2.00 & \\
\hline & 16 & $04: 45: 36.3$ & $-59: 14: 28$ & & & $5.03 \pm 0.39$ & $2.20 \pm 0.04$ & & 1.53 \\
\hline & 17 & $04: 44: 12.2$ & $-59: 23: 52$ & & & & $33.1 \pm 2.4$ & (East Lo & be) \\
\hline & & & & & & & $40.8 \pm 6.4$ & (West Lo & be) \\
\hline & 18 & $04: 44: 46.7$ & $-59: 19: 15$ & 7.74 & \pm 2.3 & & $1.55 \pm 0.10$ & & \\
\hline Source & & Optical ID & (J2000) & $\mathrm{Z}$ & $\Delta z$ & $\mathrm{~b}_{J}$ & Catalogue Name & & Remarks \\
\hline No. & & RA & & & $\left(\times 10^{-}\right.$ & & & & \\
\hline 1 & & $: 44: 14.5 \quad-59$ & $24: 53$ & .03969 & 3.30 & 18.5 & FAIRALL 0392 & & \\
\hline 2 & & $: 45: 42.5 \quad-59$ & $14: 50$ & .00433 & 0.77 & 10.28 & NGC 1672 & & SF Galaxy \\
\hline 3 & & & & & & & PMN J0443-590 & & \\
\hline 4 & & & & & & & SUMSS J044521 & $1-585926$ & \\
\hline 5 & & $: 44: 08.7 \quad-59$ & $10: 29$ & & & 19.21 & APMUKS(BJ) & & \\
\hline & & & & & & & B044320.99-5915 & 556.2 & \\
\hline 6 & & & & & & & SUMSS J044258 & 8-591923 & $\mathrm{HT}$ \\
\hline 7 & & & & & & & - & & \\
\hline 8 & & & & & & & SUMSS J044724 & $4-585406$ & \\
\hline 9 & & & & & & & PMN J0448-585 & & \\
\hline 10 & & & & & & & SUMSS J044746 & -594032 & \\
\hline 11 & & & & & & & SUMSS J044626 & s-594401 & \\
\hline 12 & & & & & & & SUMSS J044850 & -585934 & $\mathrm{HT}$ \\
\hline 13 & & $: 44: 22.2 \quad-58$ & $58: 19$ & & & 18.39 & APMUKS(BJ) & & \\
\hline & & & & & & & B044333.74-590 & 345.5 & \\
\hline 14 & & & & & & & SUMSS J044225 & -592807 & \\
\hline 16 & & & & & & & - & & \\
\hline 17 & & & & & & & - & & Double lobed \\
\hline & & & & & & & - & & radio galaxy \\
\hline 18 & & & & & & & - & & \\
\hline
\end{tabular}

Table 2.3: Properties of radio sources detected in Filament 17. Upper table describes properties observed from reduced radio maps, while lower table contains optical properties of those sources that had known optical counterparts. Catalogue name refers to either a radio or an optical entry, depending on what was available. Sources 7, 16 - 18 did not show up in any catalogue searches, though source 16 may be a part of NGC 1672, listed here as source 2. 

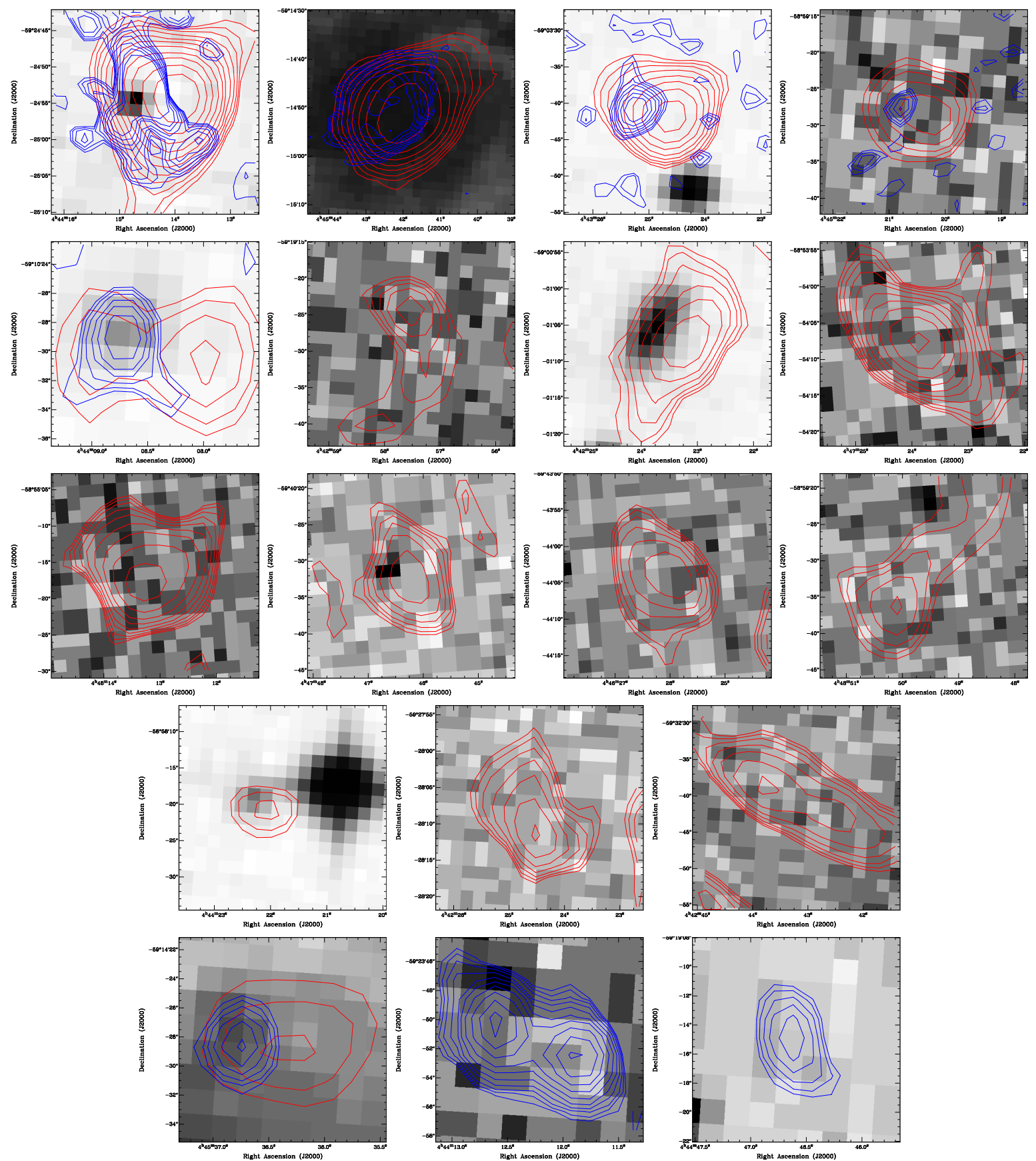

Figure 2.5: Postage stamp images of radio sources in Filament 17. Radio contours are $1.4 \mathrm{GHz}$ (red, where available) and $2.5 \mathrm{GHz}$ (blue, where available) ATCA over background optical images from the original Digitised Sky Survey. Images correspond to listed sources 1-18, counting from left to right, top to bottom. Details of the resolution and RMS noise levels are given in the text. Contours at both frequencies start at $3 \sigma$ and increase by $\sqrt{2}$ except sources $1(30 \sigma / 30 \sigma), 3(3 \sigma / 12 \sigma), 4(3 \sigma / 6 \sigma), 5(1.5 \sigma / 3 \sigma), 10(6 \sigma), 12(12 \sigma), 13(1.5 \sigma), 17(12 \sigma)$, $18(1.5 \sigma)$. This was done in an attempt to best show source structure whilst minimising artifacts. 


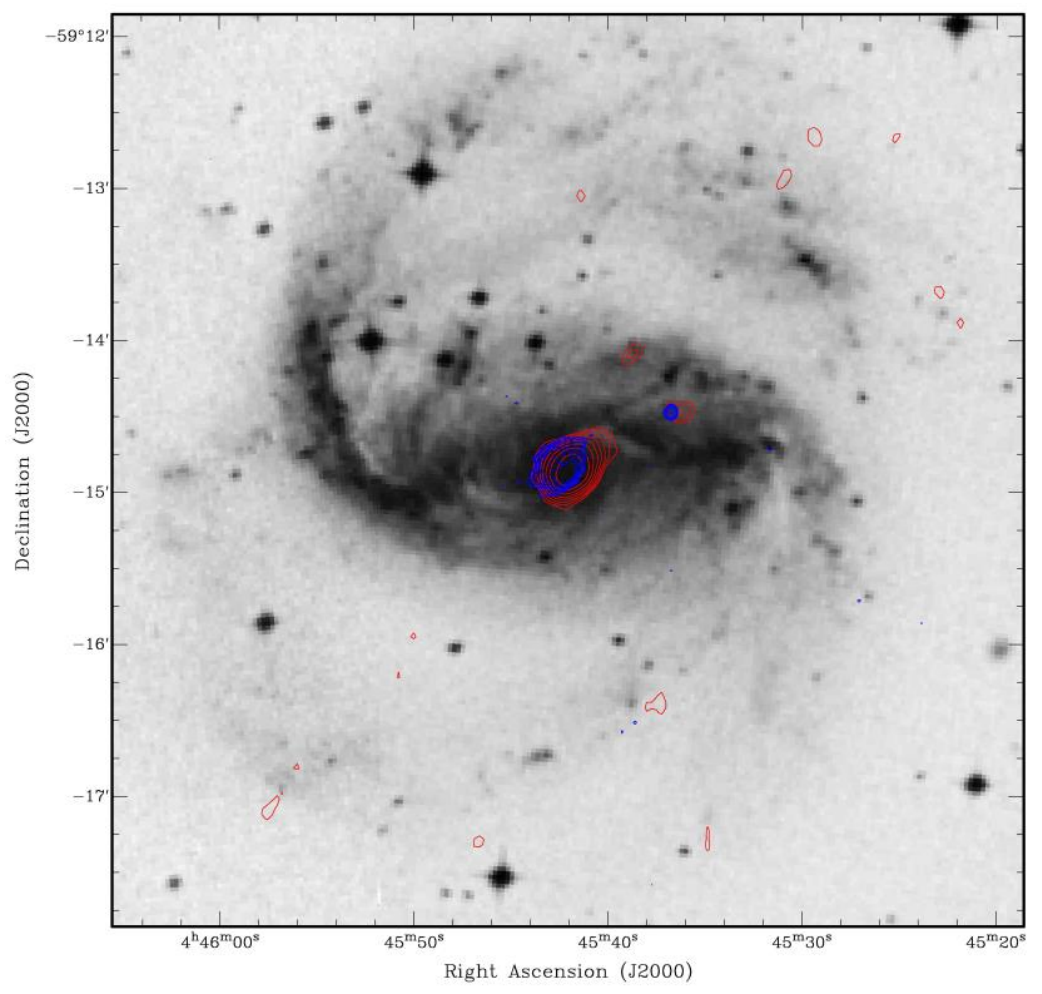

Figure 2.6: Larger image showing radio emission at 1.4 and $2.5 \mathrm{GHz}$ in the $\mathrm{SF}$ galaxy NGC1672 (Source 2), in comparison with its extent in the optical. Although little detail can be seen in the shape of the radio emission, there appears to be an elongation that aligns with the spiral arms of the host. The smaller radio source to the west is designated here as Source 16, but may also be associated with NGC1672. Radio contours at 1.4 and $2.5 \mathrm{GHz}$ start at $3 \sigma$ and increase by $\sqrt{2}$. Background image is from the original Digitised Sky Survey. Details of radio resolution, RMS and beam given in text. 
peak aligns with the optical ID. There are two possible scenarios for the observed effect. One is that we are seeing one source with a puzzling extended morphology. The other is that the $1.4 \mathrm{GHz}$ contours actually describe two sources; a weaker eastern one that coincides with the $2.5 \mathrm{GHz}$ peak and the optical ID, and a stronger western one that does not. The two sources projected onto the plane of the sky are close enough to appear as a single entity. Higher resolution observations of the source would be required to determine the cause of this phenomenon.

Of the Filament 17 population, only 4 of 18 sources had an associated optical counterpart in the DSS image (sources $1,2,5,13$ ). The two of these with catalogued redshifts (sources 1 and 2) had redshifts too low for HRS membership. The remaining Filament 17 sources are not likely to be drawn from the HRS, as at the average redshift of the supercluster $(\mathrm{z} \sim 0.06)$ they would be expected to all have associated optical IDs. This is not the case, implying that for the purposes of this thesis the data reduced for Filament 17 are too shallow to be of any use.

\subsection{Analysis of Fornax Beta Field Observational Data}

One of the very first tasks performed when considering the feasibility of this project was to check the ATCA archive for any relevant data pertaining to Filament Zones 1 and 2 (briefly mentioned in Section 2.1, dealt with further in 3.1.1). As with the rest of the HRS (see Section 2.1) almost no deep, continuum data existed for either region. The exception to this was data observed as part of the Fornax Beta Field survey, the south-eastern corner of which had some overlap with my own filament Zone 2 (between A3135 and A3145; see 3.1.1). This survey was observed on 17 April and 28-29 August 2011, utilising CABB for a central frequency of $2100 \mathrm{MHz}$ with $2 \mathrm{GHz}$ bandwidth (see Section 1.4.1 for further information about CABB). Although initially still in its proprietary period, the radio map and source catalogue, which consisted of some 1290 sources, were made available for use in this project.

As mentioned above, part of the Fornax Field does coincide with filament Zone 2. At first it was thought that these data could therefore offer some insight into filamentary sources. However, upon creating an annotation file with radio sources in their correct places, it was quickly seen that only three actually fell within Zone 2. Limiting the catalogue to only those sources with optical counterparts, the number of sources from the Fornax survey from Zone 2 dropped to one. It was therefore decided to instead use the field to test the population of deeper space. We should expect that radio sources in this region would not be strongly affected by tidal processes associated with infall. Information regarding the source counts and radio luminosity of the Fornax Beta Field would later be used as a further control, against which filament and cluster populations could be compared.

\subsubsection{Radio Source Counts}

One relatively straightforward procedure that we can perform to characterise the radio population of a field is a statistical analysis of the source counts across a range of radio powers. One appeal of a radio source count study is that, unlike the generation of a radio luminosity 


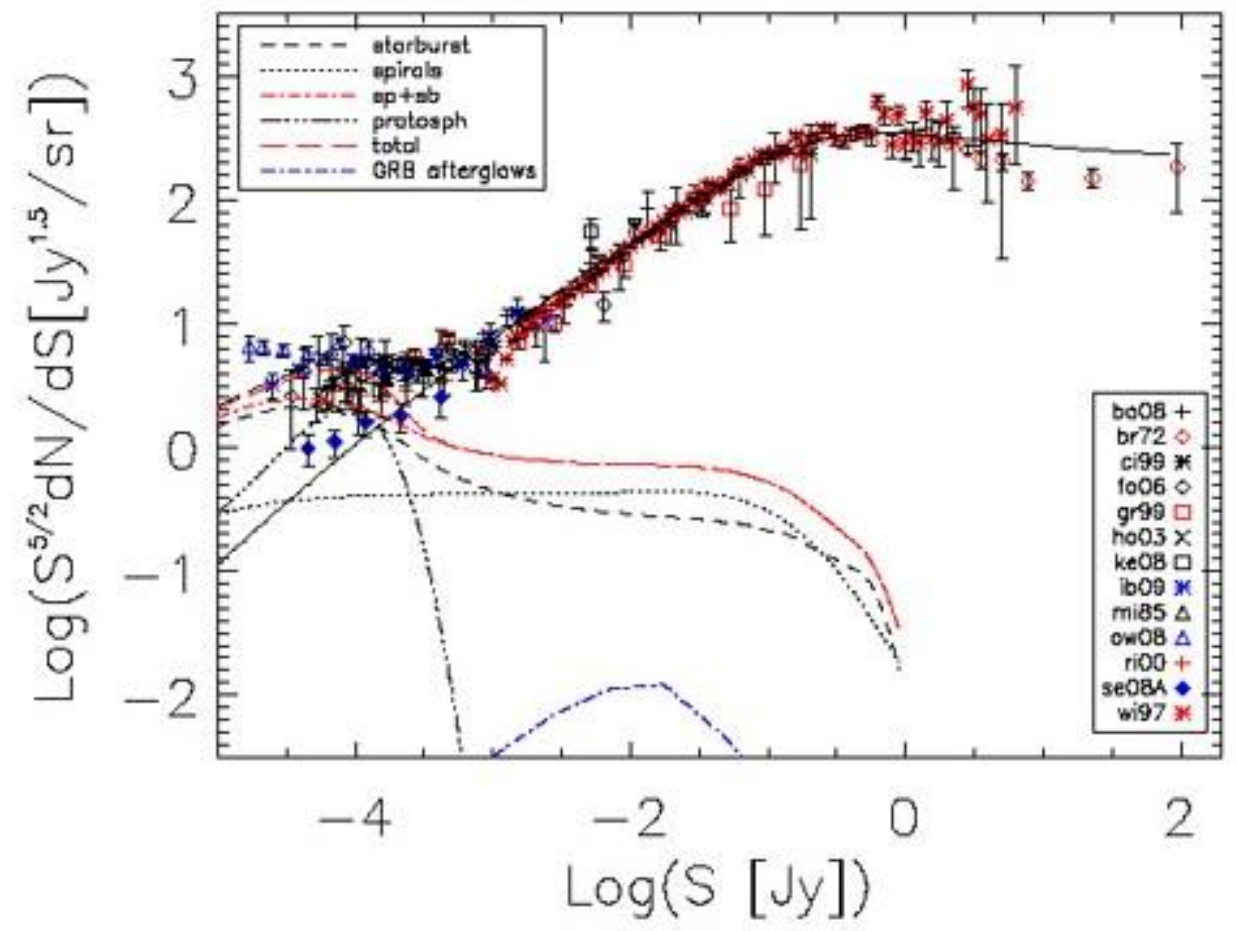

Figure 2.7: Normalized differential source counts at $1.4 \mathrm{GHz}$ from a number of different studies from (de Zotti et al. 2010). While full explanation can be found in that paper, the plot was included here simply to illustrate the divergence of different source count models below $\sim 1 \mathrm{mJy}$. Above this flux level the different models show strong agreement, but in the low flux regime we are faced with the problem of no canonical source count study against which to compare our own findings.

function (RLF, see below), this does not require the retrieval of any optical data for our radio sources. This is convenient, and also allows us to retain a much larger sample catalogue, providing more robust results. An ideal comparison population is that presented in Prandoni et al. (2001). Prandoni et al. (2001) surveyed an area $\sim 26$ square degrees in extent, using the ATCA at $1.4 \mathrm{GHz}$. The study produced a sample of 2960 sources, complete across the flux range $0.7 \leq \mathrm{S}_{1.4} \leq 2 \mathrm{mJy}$. Although a number of studies have probed source counts to lower flux limits than Prandoni et al., below $\sim 1$ mJy cosmic variance results in disagreement between them (see Fig. 2.7). As well as this, correctly accounting for the scaling on different telescopes is a complex and time consuming process, outside the scope of this study. The source count profile of Prandoni et al. was drawn from ATCA data in continuum mode, meaning that direct comparison with our data is appropriate providing that we correct for the observing frequency, as below.

In comparison with Prandoni et al., the Fornax Beta Field catalogue consists of 1290 sources detected at $5 \sigma(\mathrm{rms} \approx 0.14 \mathrm{mJy})$. All catalogued sources have integrated fluxes above 0.69 $\mathrm{mJy}$, and inhabit an area on the sky covering $\sim 49$ square degrees.

The Fornax observations utilised the CABB-upgraded ATCA, with a central observing fre- 
quency of $1588 \mathrm{MHz}$, and were undertaken as part of the commissioning process for ASKAP. During reduction the band, $\Delta \nu=512 \mathrm{MHz}$ was split into four subbands. Antenna 6 was not used. The synthesised beam size was $1.42^{\prime} \times 0.6^{\prime}$, and the $(\mathrm{J} 2000)$ coordinates of the map centre were 03:29:60, -36:08:24.9.

Data and the reduced maps and catalogue from these observations were provided for use in this study by Franzen. In order to properly compare the two samples, this difference in frequency had to be accounted for. To do this the $1588 \mathrm{MHz}$ fluxes of Fornax Field sources were scaled to $1376 \mathrm{MHz}$ using the spectral index equation:

$$
S \propto \nu^{-\alpha}
$$

Therefore:

$$
\frac{S_{2}}{S_{1}}=\exp \left[\alpha \ln \left(\frac{\nu_{1}}{\nu_{2}}\right)\right]
$$

Here the spectral index $\alpha$ is defined as positive. In these calculations the default figure of 0.7 was used. Once the flux values had been corrected (at this point the minimum integrated source flux was $0.77 \mathrm{mJy}$ ), the data was binned so that each bin had width $\Delta \mathrm{S}=\mathrm{S} \times \log (2.5)$. A $\log N / \log S$ plot of the differential source counts for the Fornax sample is presented in Figure 2.8, along with the source counts from Prandoni et al. (2001) for comparison.

Below around $5 \mathrm{mJy}$ our sample is clearly incomplete. This corresponds to a radio lower of $\log \mathrm{P}_{1.4}=22.6$ (assuming Eqn 1.5 with $\mathrm{z}=0.0623$ for all sources) and implies that the Fornax Field survey, like the majority of previous studies outlined in Section 1.3, only probes the upper limit of the star-formation-dominated regime. Above $5 \mathrm{mJy}$ the Fornax source counts seem to closely follow those of Prandoni et al., though with an overall deficit in source counts for all bins below 100 mJy. Certainly no significant excess is evident in the population. Of course an excess of strictly low-powered star forming galaxies would take place below the completeness limit of the Fornax Field profile, but there are no features on the plotted line that would lead us to expect such an increase at lower flux. One or more of several mechanisms could contribute to the observed overall source count deficit. However, without further multiwavelength observations of the Fornax Field population no robust conclusions can be made.

\subsubsection{Radio Luminosity Function}

Before any analysis of the radio luminosity of sources in the field could be undertaken, it was necessary to attach to the radio catalogue an associated optical catalogue for as many sources as possible. In particular, the optical magnitudes were required. This optical data was gathered from two sources. First, the entire radio catalogue was submitted to the NASA Extragalactic Database ${ }^{4}$ as a batch query. A radius of $0.03 \operatorname{arcmin}(\sim 2 \operatorname{arcsec})$ was chosen to define optical/radio correlation. This search returned 68 optical sources with known magnitudes.

\footnotetext{
${ }^{4}$ http://ned.ipac.caltech.edu/
} 


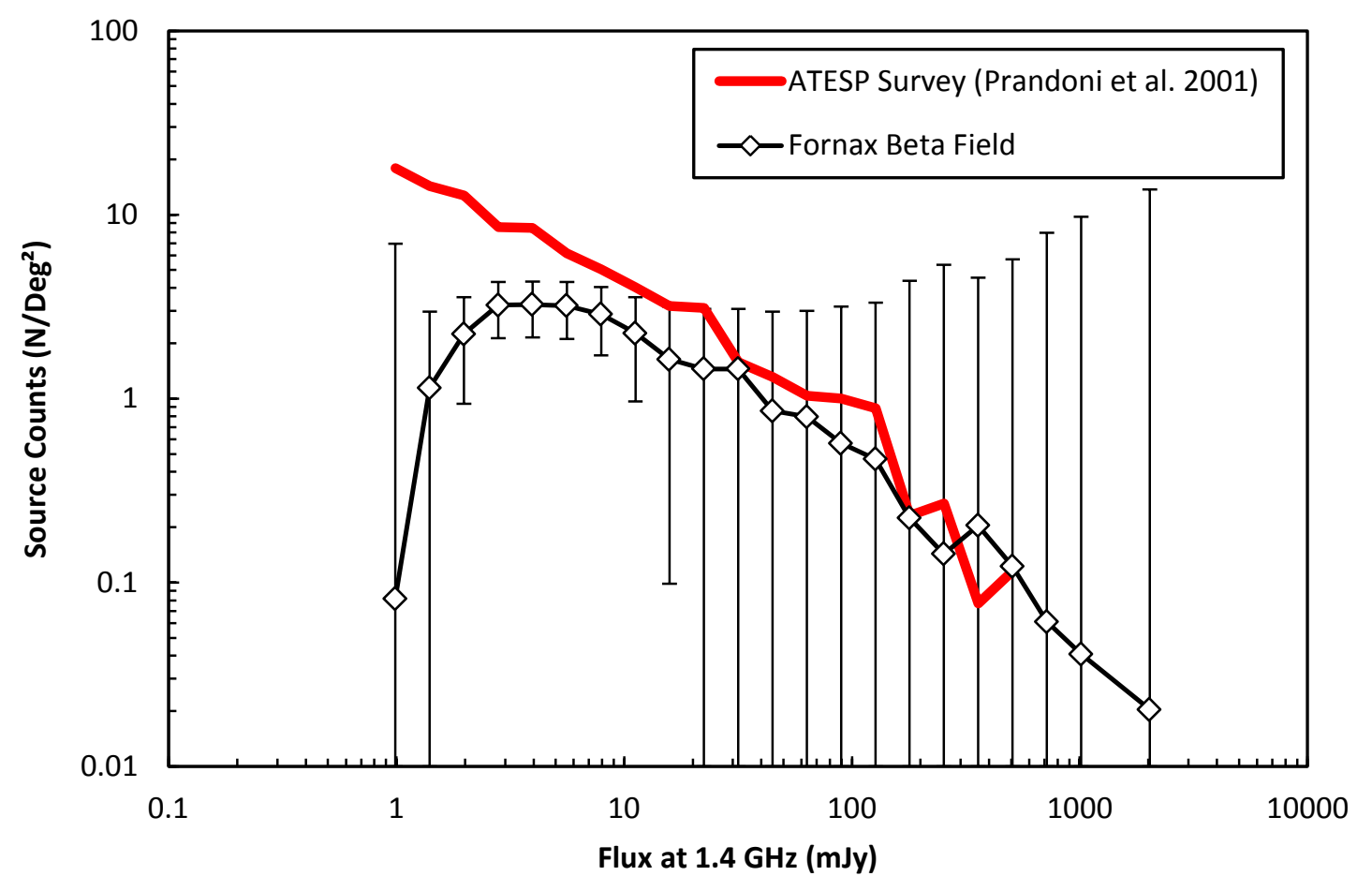

Figure 2.8: Differential source counts for Fornax Beta Field radio population, compared with those of the ATESP survey (Prandoni et al. 2001). For the former population $2 \sigma$ Poissonian error bars have been added to give an estimate of uncertainty. We see strong agreement here between the two profiles above 10 mJy, while below $\sim 5$ mJy $(\log \mathrm{P} \approx 22.6)$ the Fornax Field sample is clearly incomplete. At this point the Fornax Field profile drops away steeply from the line of Prandoni et al. (2001). 
In addition to the NED batch query, an optical source catalogue was obtained from SuperCOSMOS $^{5}$ for the entire area covered by the Fornax Beta Field. This catalogue was substantial. Once both non-galaxy sources and sources within 1.0 degrees of a cluster centre $\left(\sim R_{200}\right)$ were removed, there remained around 130000 optical sources to deal with. These were limited by incorporating a matching script to keep only those optical sources found within 2 arcsec of a catalogued radio source. This script provided 63 radio/optical correlations. In no case did a radio source have multiple optical sources within the 2 arcsec radius. However, the majority of radio/optical correlations found using SuperCOSMOS were for the same sources returned by NED. The total number of unique radio/optical matches across the two searches was 83 .

Before we undertake any analysis of radio luminosity functions, it is worth considering for a moment the extend to which our optical/radio catalogue represents the entire radio population. Following the treatment of Venturi et al. (2000) we can visually assess the reliability of the subpopulation of sources for which we have optical counterparts. The two populations are presented in Fig. 2.9 as a histogram pair, with sources binned by flux. Without needing to undertake any rigorous statistical analysis, we can see that the two populations display very similar flux distributions, and thus the optical/radio catalogue is an appropriate proxy by which to analyse flux-based properties of the full Fornax Beta Field population.

The univariate integral RLF is shown in Fig. 2.10, with data for a range of Johnston blue (optical) magnitude limits from 18.05 to 22 . The plot describes the likelihood that, if we limit our optical magnitude to some value, a galaxy will be found with a radio power $\geq$ $\log \mathrm{P}_{1.4}$. It can be seen from the figure that as we lower the limit on optical magnitude (a lower magnitude value corresponds to a brighter optical source), the likelihood of finding such a radio power increases. That brighter optical galaxies are more likely to host radio emission is a well known phenomenon (Ledlow and Owen 1996; Venturi et al. 1997). The radio luminosity functions in the figure are consistent with this. Notably, we also see some evidence of an increase in the RLFs at $\log \mathrm{P} \approx 24.5$ for magnitude limits 18.05 and 19 , in agreement with that seen in the radio/optical population histogram in Fig. 2.9.

The bivariate RLF for any field is not particularly enlightening unless is can be compared to those of other fields, and any similarities or differences noted. The current benchmark at the time of writing is the cluster sample RLF presented in Ledlow and Owen (1996), hereafter LO96. In order to make a meaningful comparison between samples, our radio catalogue had to be corrected for the different cosmologies.

Also, optical magnitudes above (fainter than) $\mathrm{b}_{J}=18.05$ were discarded, in keeping with the $\mathrm{M}_{R} \leq-20.5$ magnitude limit of the LO96 sample. The conversion here assumes that $\mathrm{b}_{J}=$ B - 0.2(B-V) (Fukugita et al. 1995). After applying these constraints to our catalogue, we were left with a sample of fifteen radio sources that also exhibited optical emission. The total optical population of the region consisted of 949 sources. However, the fraction of these that lie within an appropriate redshift range for comparison with HRS samples, and are also early type galaxies, is significantly less than $100 \%$. Following the assumptions of Johnston-Hollitt et al. (2008) the respective values attributed to these two limiting factors were 0.74 and 0.77. The optical population used as the basis of our bivariate RLF calculation therefore consisted of 541 sources that were both early-type and of a comparable redshift to the HRS. Whether

\footnotetext{
${ }^{5}$ http://www-wfau.roe.ac.uk/sss/index.html
} 


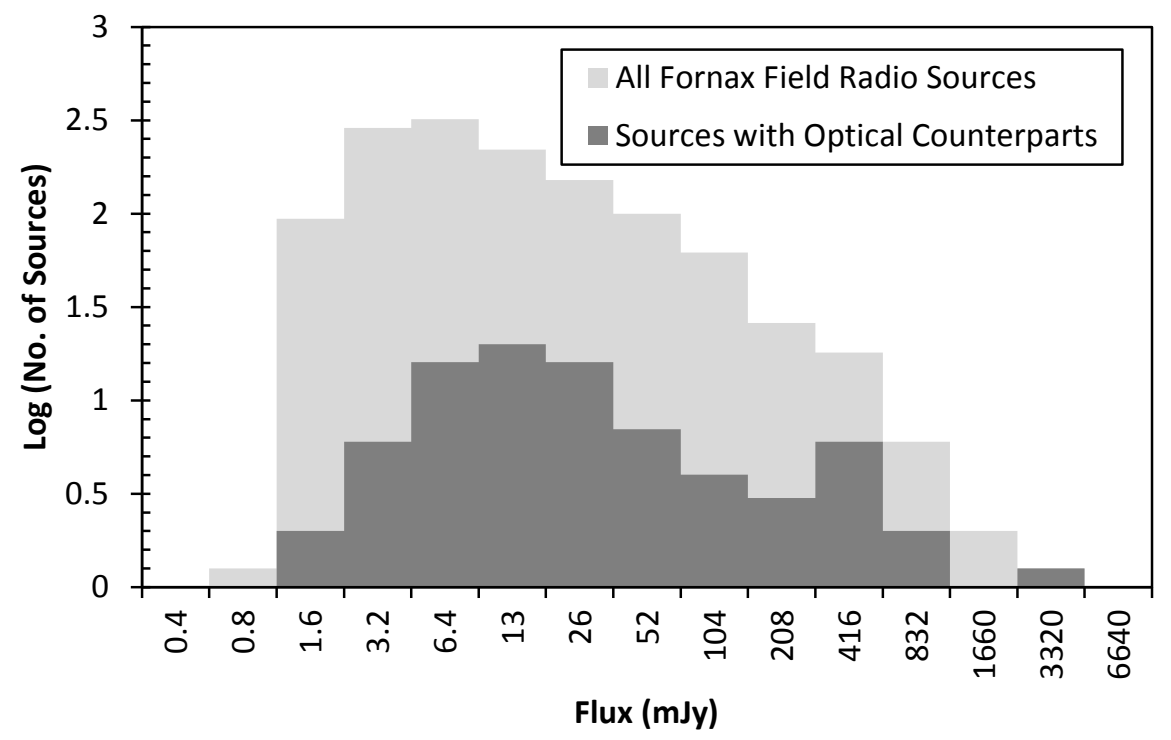

Figure 2.9: Comparison of the fluxes of sources in the Fornax Beta Field catalogue. The full radio catalogue distribution (light grey) is compared with that of radio sources with associated optical magnitudes, from NED and SuperCOSMOS online catalogues (dark grey). Bins with a single source have been artificially boosted to a little over 0 (as $\log [1]=0)$ to distinguish them from bins with no sources. The minor peak at $S \sim 400$ mJy consists of 6 sources, with 3 and 2 to the left and right respectively. If this peak is significant, we should expect to see a similar increase in the RLF at this point, which corresponds to $\log \mathrm{P} \approx 24.5$. 


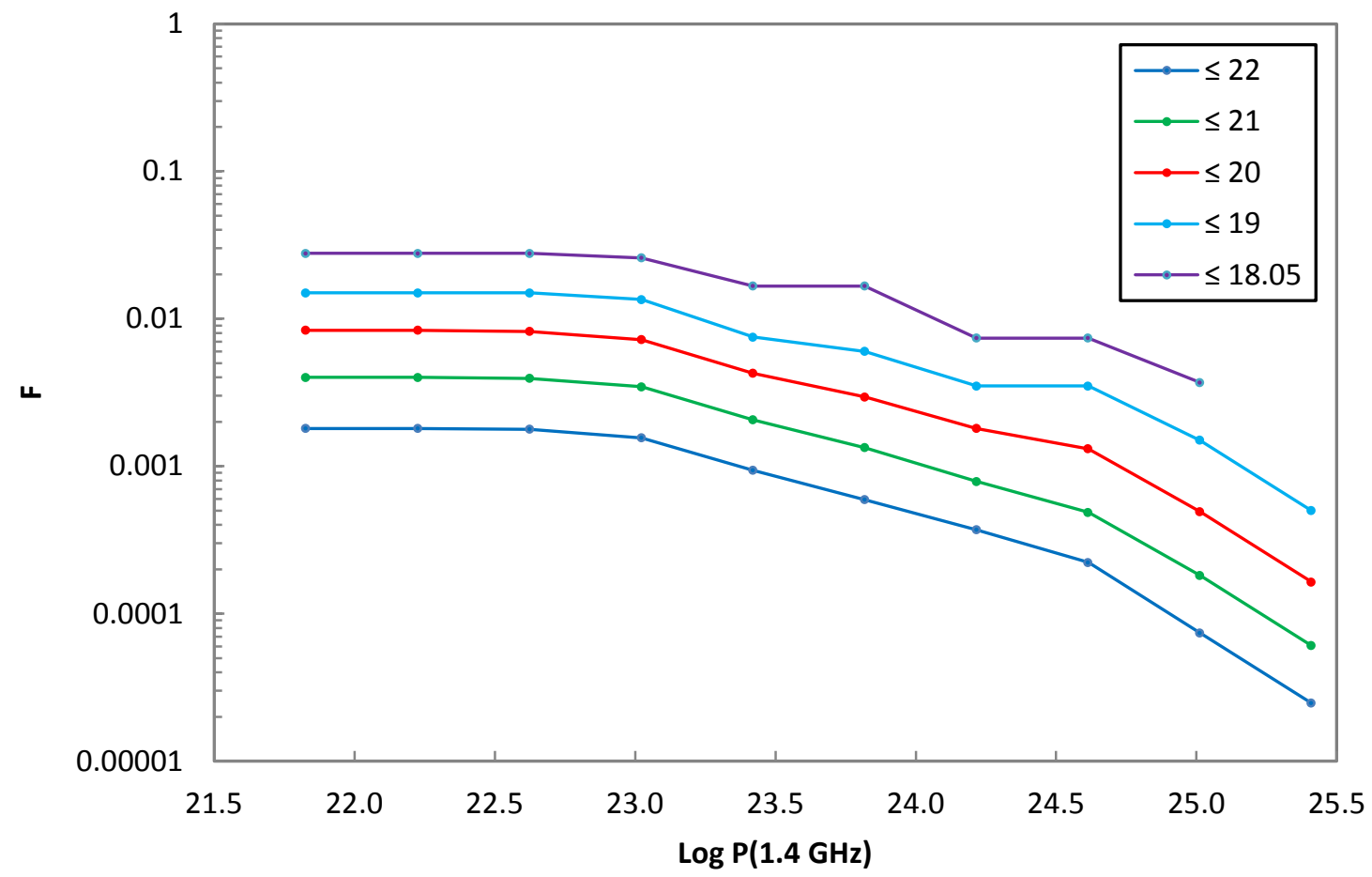

Figure 2.10: Univariate radio luminosity function for Fornax Beta Field population. Plot shows the likelihood that, for some optical magnitude cutoff, an object will be found with a radio power greater than $\mathrm{P}$. Radio power has been corrected to $1.4 \mathrm{GHz}$ assuming a spectral index of 0.7 for all sources. Magnitude limits are Johnston blue magnitudes from SuperCOSMOS and NED. The slight increase in the top two RLF lines at $\log \mathrm{P} \approx 24.5$ is consistent with that seen in Fig. 2.9, implying that this is a feature of the optical, rather than the radio, population. 


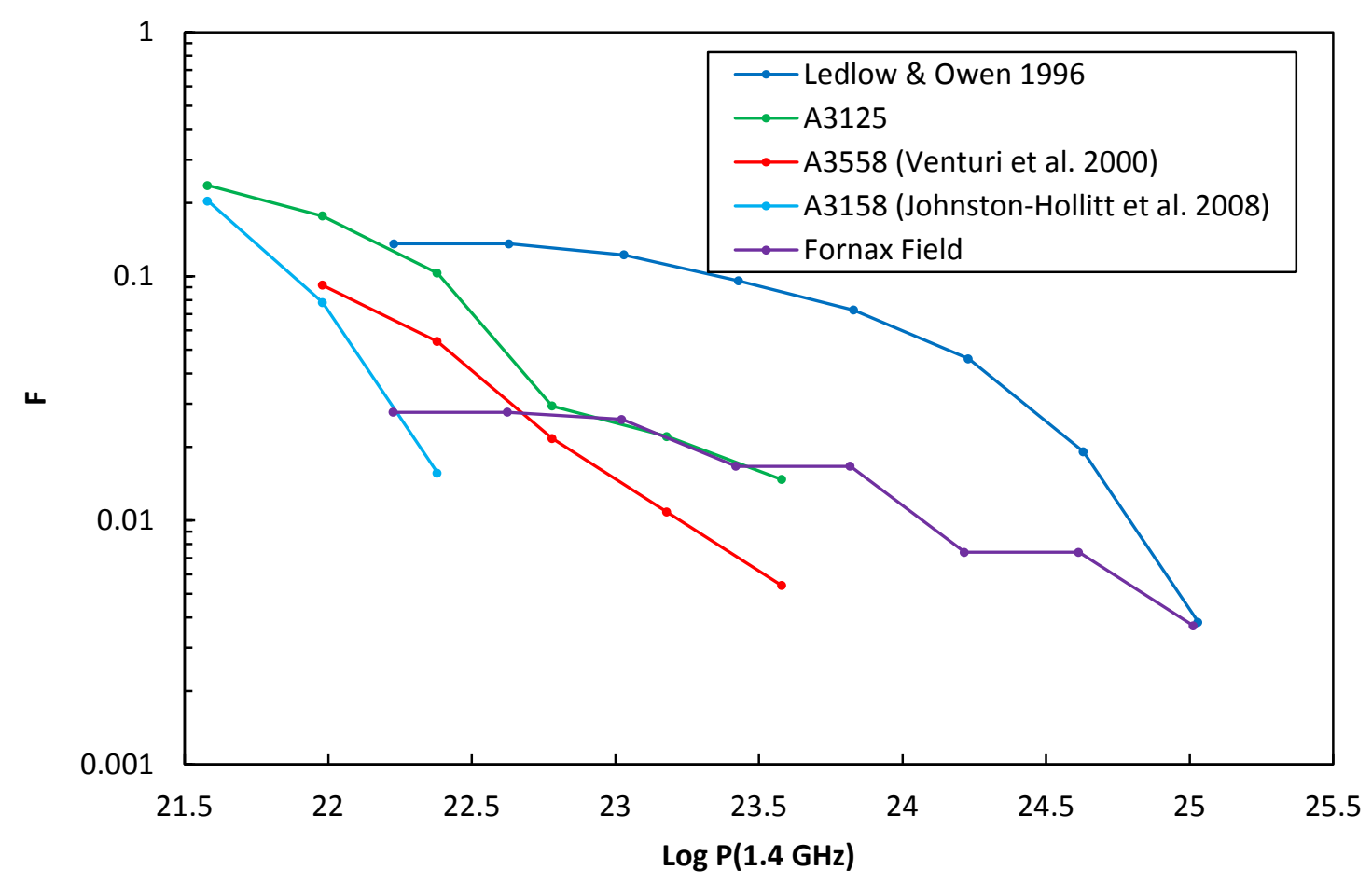

Figure 2.11: Bivariate radio luminosity function for the Fornax Beta field population, scaled to $1.4 \mathrm{GHz}$ and with an optical magnitude cutoff of $\mathrm{b}_{J}=18.05$ applied, in keeping with the brightness limit in Ledlow and Owen (1996). The Fornax Field RLF seems to best agree with the line drawn from the cluster population of A3125 (Johnston-Hollitt \& Grimwood, in prep.).

the fraction of early-type galaxies in the Fornax Beta Field ought to be similar to that seen in cluster/supercluster populations is as yet unknown. The Fleenor study of inter-cluster regions of the HRS Fleenor et al. (2005) adopted a figure of $74 \%$. This may imply that the higher the concentration of matter, the more likely we are to find early-types; thus in the Fornax Beta Field we may expect a fraction rather lower than for the clusters. Just how much lower, is unclear. At the least, the figure used provides us with a first-order approximation for the bivariate RLF of the field.

The bivariate RLF presented in Figure 2.11 allows us to compare the radio population of the Fornax Beta Field with a number of other sample populations. The data upon which the Fornax Field RLF is based, is presented in Table 2.4. The primary comparison is of course the previously mentioned LO96 sample Ledlow and Owen (1996). Also displayed on the plot are RLFs for A3558 Venturi et al. (2000), A3158 Johnston-Hollitt et al. (2008) and A3125 (Johnston-Hollitt \& Grimwood, in prep.). It can be seen in the plot that compared to the cluster samples, the Fornax Beta Field appears to show a deficit of low-powered radio sources. For radio powers $\log \mathrm{P}_{1.4} \leq 23 \mathrm{WHz}^{-1}$ the the probability of finding radio sources brighter than $\log \mathrm{P}_{1.4}$ levels off at around 0.012. This shape is similar to that of LO96, but here the value of $\mathrm{F}$ is around $10 \%$ of the figure obtained for that sample. The other three cluster 


\begin{tabular}{cccccc}
\hline$\Delta \log \mathrm{P}_{1.4}$ & $\Delta \mathrm{S}_{1.4}$ & Fractional BRLF & \multicolumn{3}{c}{ Integral BRLF } \\
\hline $21.43-21.83$ & $0.33-0.82$ & $0 / 540$ & 0 & $15 / 540$ & 0.02778 \\
$21.83-22.23$ & $0.82-2.06$ & $0 / 540$ & 0 & $15 / 540$ & 0.02778 \\
$22.23-22.62$ & $2.06-5.15$ & $1 / 540$ & 0.00185 & $15 / 540$ & 0.02778 \\
$22.62-23.02$ & $5.15-12.9$ & $5 / 540$ & 0.00926 & $14 / 540$ & 0.02593 \\
$23.02-23.42$ & $12.9-32.2$ & $0 / 540$ & 0 & $9 / 540$ & 0.01667 \\
$23.42-23.82$ & $32.2-80.5$ & $5 / 540$ & 0.00926 & $9 / 540$ & 0.01667 \\
$23.82-24.22$ & $80.5-201$ & $0 / 540$ & 0 & $4 / 540$ & 0.00740 \\
$24.22-24.61$ & $201-503$ & $2 / 540$ & 0.00370 & $4 / 540$ & 0.00740 \\
$24.61-25.01$ & $503-1260$ & $2 / 540$ & 0.00370 & $2 / 540$ & 0.00370 \\
$25.01-25.41$ & $1260-3140$ & $0 / 540$ & 0 & $0 / 540$ & 0 \\
\hline
\end{tabular}

Table 2.4: Details of the Bivariate RLF for the Fornax Beta Field population.

RLF lines continue to increase over the range $\log \mathrm{P}_{1.4} \leq 23 \mathrm{WHz}^{-1}$, showing a substantial excess of low-powered radio galaxies - in the cases of A3125 and A3158 this goes beyond the level one might expect from extrapolating the LO96 line into a comparable low-power regime. This excess is not evident in the Fornax sample. It was mentioned above that the fraction of early-type galaxies for non-cluster fields is not a well-known value. If this fraction was significantly lower than the $77 \%$ adopted, the probabilities at all points on our Fornax Field RLF would be boosted. However, unless we were inaccurate by a factor of ten (i.e. if an appropriate fraction should actually be $\sim 8 \%$ ) our RLF would still fall well below the LO96 line. Even this would not affect the overall shape of the Fornax RLF.

According to Miller and Owen (2002), a division between AGN and star-forming behaviour in clusters occurs around $\log \mathrm{P}_{1.4}=22.8 \mathrm{WHz}^{-1}$. This figure describes the populations of rich-clusters with high early-type fractions. A general value for the field is probably closer to 23.0 $\mathrm{WHz}^{-1}$ (Condon et al. 2002; Mauch and Sadler 2007). Referring again to Figure 2.11, we see that such a division in the Fornax Field sample corresponds to the power at which the probability levels off. This would suggest that the Fornax Field is dominated by AGN, with very few star-forming galaxies having been detected, at least at this flux level. Referring to Eqn. 1.4, the power level of $\log \mathrm{P}_{1.4} \sim 23$ at which the RLF for the Fornax Field population levels off corresponds to a star formation rate of $\mathrm{SFR}\left(\mathrm{M} \geq 5 \mathrm{M}_{\odot}\right) \approx 25 \mathrm{M}_{\odot} / \mathrm{yr}$. 


\section{Chapter 3}

\section{ATCA Observations of Zone 1 and Zone 2 Filaments}

\subsection{Radio Observations and Reduction}

The following section details the observational parameters and subsequent reduction of new $2.1 \mathrm{GHz}$ ATCA observations of the filaments in Zones $1 \& 2$.

\subsubsection{Region Selection}

Detailed optical analysis of the HRS presented in Fleenor (2006) defines a filament between the A3125/A3128 complex and the neighbouring cluster A3158 (Fig. 3.1). Earlier ATCA observations by Johnston-Hollitt et al. (2008) and Johnston-Hollitt \& Grimwood (in prep.) of the clusters A3158, A3125 and A3128, which lie at the heart of the HRS, had explored the radio luminosity functions and source counts in these clusters. The filament between them, initially designated Filament 19 in Table 2.1 and thereafter referred to as Zone 1, was therefore an ideal place to conduct a more detailed study.

The field of view of a radio telescope is given by the formula

$$
\theta_{f o v}=\frac{k \lambda}{d}
$$

Where $\lambda$ is the wavelength being observed ( $16 \mathrm{~cm}$ for $2.1 \mathrm{GHz}$ observations), $\mathrm{d}$ is the dish size $(22 \mathrm{~m}$ for ATCA $)$, and $\mathrm{k}$ is a constant that describes the aperture shape $(\mathrm{k} \approx 1.22$ for circular apertures). This means that at $2.1 \mathrm{GHz}$ our field of view (diameter) will be $\theta_{\text {fov }}=29.7^{\prime}$. Of course, our region is bigger than this. The standard method for observing a field larger than a single pointing is to observe several pointings spaced evenly across the field, and to combine the data into a mosaic. To obtain even coverage across the mosaic it is desirable to adopt a regular geometric pattern for the pointing centres. The two most common arrangements are rectangular: 


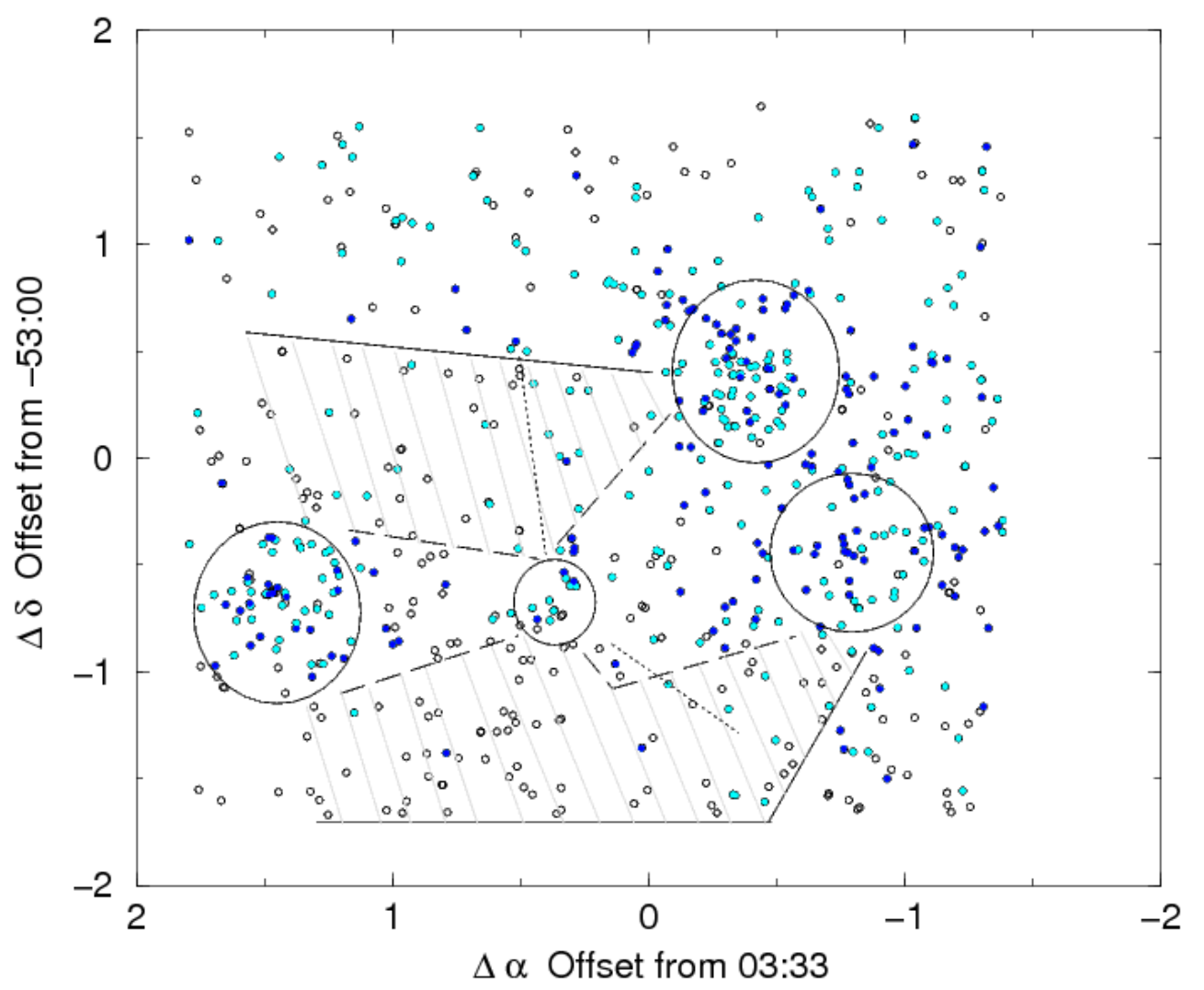

Figure 3.1: Primary region of interest bounded by A3158 (left), and the A3125/A3128 complex (right). Light filled circles show galaxies with known redshifts. Darker filled circles correspond to galaxies with cz between 17,250 and $18,575 \mathrm{kms}^{-1}$. Dashed lines indicate the extent of the filamentary structure connecting these clusters. The smaller circle shows the position of the non-rich cluster APMCC421. (Fleenor 2006) 


$$
\theta_{\text {Rect }}=\frac{\lambda}{2 d}
$$

And hexagonal:

$$
\theta_{\text {Hex }}=\frac{\lambda}{\sqrt{3} d}
$$

Of these the latter covers area most efficiently, so was used as the pointing arrangement for the observations in this project. Although it is called a hexagonal grid, it is really generated by tessellating equilateral triangles. For our purposes these were triangles with sides 12.9'.

One hour's observation with CABB allows us to achieve a sensitivity of $0.045 \mathrm{mJy}$ (with $3 \sigma$ detection). This allows us to theoretically detect star formation at a rate as low as 0.094 solar masses per year (see Eqns. 1.4 and 1.5). This level $\left(\sim 0.1 \mathrm{M}_{\odot} / \mathrm{yr}\right)$ was chosen as the detection goal for this project. So for every pointing in our mosaic, one hour of ATCA time was requested (plus calibration time, $\sim 20 \%$ ) because the field would require more than 12 pointings, this time would provide full hour-angle coverage as the instrument rotated through $180^{\circ}$.

In order to observe the Zone 1 field, a 28-pointing mosaic was proposed for the April 2012 semester using the ATCA at $2.1 \mathrm{GHz}$. The layout of this mosaic is presented in Fig. 3.2, which also includes positions of the previously observed pointings from project C995 (JohnstonHollitt \& Grimwood, in prep; see Table 3.1).

A multiwavelength campaign using radio, optical and X-ray data over A3135 confirmed the existence of a filament between this cluster and A3145. Pratley et al. (2013) also present archival ATCA observations of A3135 (project code C959, PI Saripalli - see Table 3.2). This region, Filament 20 in Table 2.1 and Zone 2 thereafter, was thus also selected for deep ATCA observations.

Zone 2 could be covered by mosaic observation on a similar scale, using the ATCA at 2.1 GHz. This time the region would require 27 pointings to cover the notional filament region between the two cluster edges. As mentioned above, A3135 had been observed previously at 20cm (Pratley et al. 2013), but A3145 and the filamentary region between the two had not, to any significant depth. The slight exception is the Parkes $1.4 \mathrm{GHz}$ observations from projects P593 (PI R Braun) and P617 (PI M Wolleben), observed in September 2008. These pointings do fall in the Zone 2 filament region (as shown in Fig. 3.3), but are extremely shallow. Each pointing had a scan time of only 5.3 minutes, meaning that adding these data to our own observations would not be useful.

Again for Zone 2 observing time equating to one hour per pointing plus overheads, was requested to provide both sufficient depth to probe star formation at the $\sim 0.1 \mathrm{M}_{\odot} / \mathrm{yr}$ level, and hour angle coverage.

Repeated analysis using the radio population of a second filament was expected to show similar trends as exhibited by Zone 1 . Even if this were to be the case, the addition of this data would solidify any conclusions, allowing us to largely avoid 'cosmic variance' arguments. 


\begin{tabular}{|c|c|c|c|c|c|c|}
\hline $\begin{array}{c}\text { Project } \\
\text { Code }\end{array}$ & Date(s) & $\begin{array}{l}\text { Observing } \\
\text { Time (min) }\end{array}$ & $\begin{array}{c}\text { Baseline } \\
\text { Config. }\end{array}$ & $\begin{array}{c}\text { Frequency } \\
\quad(\mathrm{MHz})\end{array}$ & $\begin{array}{r}\text { Pointing } \\
\text { RA } \\
\end{array}$ & $\begin{array}{l}\text { Centre } \\
\text { Dec (J2000) }\end{array}$ \\
\hline \multirow[t]{30}{*}{ C995 } & $10 / 12 / 2001$ & 522.7 & $6 \mathrm{~A}$ & 1344,1432 & 03:32:14.88 & $-53: 05: 21.6$ \\
\hline & $10 / 12 / 2001$ & 522.7 & $6 \mathrm{~A}$ & 1344,1432 & 03:32:14.88 & $-53: 22: 02.4$ \\
\hline & 09/12/2001 & 482.4 & $6 \mathrm{~A}$ & 1344,1432 & 03:32:14.88 & $-53: 05: 21.6$ \\
\hline & 09/12/2001 & 482.4 & $6 \mathrm{~A}$ & 1344,1432 & 03:32:14.88 & $-53: 22: 02.4$ \\
\hline & 08/12/2001 & 120.8 & $6 \mathrm{~A}$ & 1344,1432 & 03:32:14.88 & $-53: 05: 21.6$ \\
\hline & $08 / 12 / 2001$ & 120.8 & $6 \mathrm{~A}$ & 1344,1432 & 03:32:14.88 & $-53: 22: 02.4$ \\
\hline & $29 / 11 / 2001$ & 522.4 & $6 \mathrm{D}$ & 1344,1432 & 03:32:14.88 & $-53: 22: 02.4$ \\
\hline & $28 / 11 / 2001$ & 522.4 & $6 \mathrm{D}$ & 1344,1432 & 03:32:14.88 & $-53: 05: 21.6$ \\
\hline & $27 / 11 / 2001$ & 602.9 & $6 \mathrm{D}$ & 1344,1432 & 03:32:14.88 & $-53: 05: 21.6$ \\
\hline & $27 / 11 / 2001$ & 602.9 & $6 \mathrm{D}$ & 1344,1432 & $03: 32: 14.88$ & $-53: 22: 02.4$ \\
\hline & $21 / 11 / 2001$ & 361.9 & $1.5 \mathrm{D}$ & 1344,1432 & 03:32:14.88 & $-53: 05: 21.6$ \\
\hline & $21 / 11 / 2001$ & 361.9 & $1.5 \mathrm{D}$ & 1344,1432 & 03:32:14.88 & $-53: 22: 02.4$ \\
\hline & $20 / 11 / 2001$ & 301.3 & $1.5 \mathrm{D}$ & 1344,1432 & 03:32:14.88 & $-53: 05: 21.6$ \\
\hline & $20 / 11 / 2001$ & 301.3 & $1.5 \mathrm{D}$ & 1344,1432 & 03:32:14.88 & $-53: 22: 02.4$ \\
\hline & $20 / 11 / 2001$ & 301.3 & $1.5 \mathrm{D}$ & 1344,1432 & 03:32:14.88 & $-53: 38: 43.2$ \\
\hline & $20 / 11 / 2001$ & 301.3 & $1.5 \mathrm{D}$ & 1344,1432 & 03:34:05.76 & $-53: 38: 43.2$ \\
\hline & $20 / 11 / 2001$ & 301.3 & $1.5 \mathrm{D}$ & 1344,1432 & 03:34:05.76 & $-53: 22: 02.4$ \\
\hline & $20 / 11 / 2001$ & 301.3 & $1.5 \mathrm{D}$ & 1344,1432 & 03:34:05.76 & $-53: 05: 21.6$ \\
\hline & $20 / 11 / 2001$ & 301.3 & $1.5 \mathrm{D}$ & 1344,1432 & 03:35:56.64 & $-53: 05: 21.6$ \\
\hline & $20 / 11 / 2001$ & 301.3 & $1.5 \mathrm{D}$ & 1344,1432 & 03:35:56.64 & $-53: 22: 02.4$ \\
\hline & $20 / 11 / 2001$ & 301.3 & $1.5 \mathrm{D}$ & 1344,1432 & 03:35:56.64 & $-53: 38: 43.2$ \\
\hline & $19 / 11 / 2001$ & 241.1 & $1.5 \mathrm{D}$ & 1344,1432 & 03:32:14.88 & $-53: 05: 21.6$ \\
\hline & $19 / 11 / 2001$ & 241.1 & $1.5 \mathrm{D}$ & 1344,1432 & 03:32:14.88 & $-53: 22: 02.4$ \\
\hline & $19 / 11 / 2001$ & 241.1 & $1.5 \mathrm{D}$ & 1344,1432 & 03:32:14.88 & $-53: 38: 43.2$ \\
\hline & $19 / 11 / 2001$ & 241.1 & $1.5 \mathrm{D}$ & 1344,1432 & 03:34:05.76 & $-53: 38: 43.2$ \\
\hline & $19 / 11 / 2001$ & 241.1 & $1.5 \mathrm{D}$ & 1344,1432 & 03:34:05.76 & $-53: 22: 02.4$ \\
\hline & $19 / 11 / 2001$ & 241.1 & $1.5 \mathrm{D}$ & 1344,1432 & 03:34:05.76 & $-53: 05: 21.6$ \\
\hline & $19 / 11 / 2001$ & 241.1 & $1.5 \mathrm{D}$ & 1344,1432 & 03:35:56.64 & $-53: 05: 21.6$ \\
\hline & $19 / 11 / 2001$ & 241.1 & $1.5 \mathrm{D}$ & 1344,1432 & 03:35:56.64 & $-53: 22: 02.4$ \\
\hline & $19 / 11 / 2001$ & 241.1 & $1.5 \mathrm{D}$ & 1344,1432 & 03:35:56.64 & $-53: 38: 43.2$ \\
\hline
\end{tabular}

Table 3.1: Previous observations of the Zone 1 filament region, located between the clusters A3158, A3125 and A3128. PI R. Hunstead, Project C995 (Johnston-Hollitt \& Grimwood, in prep.) 


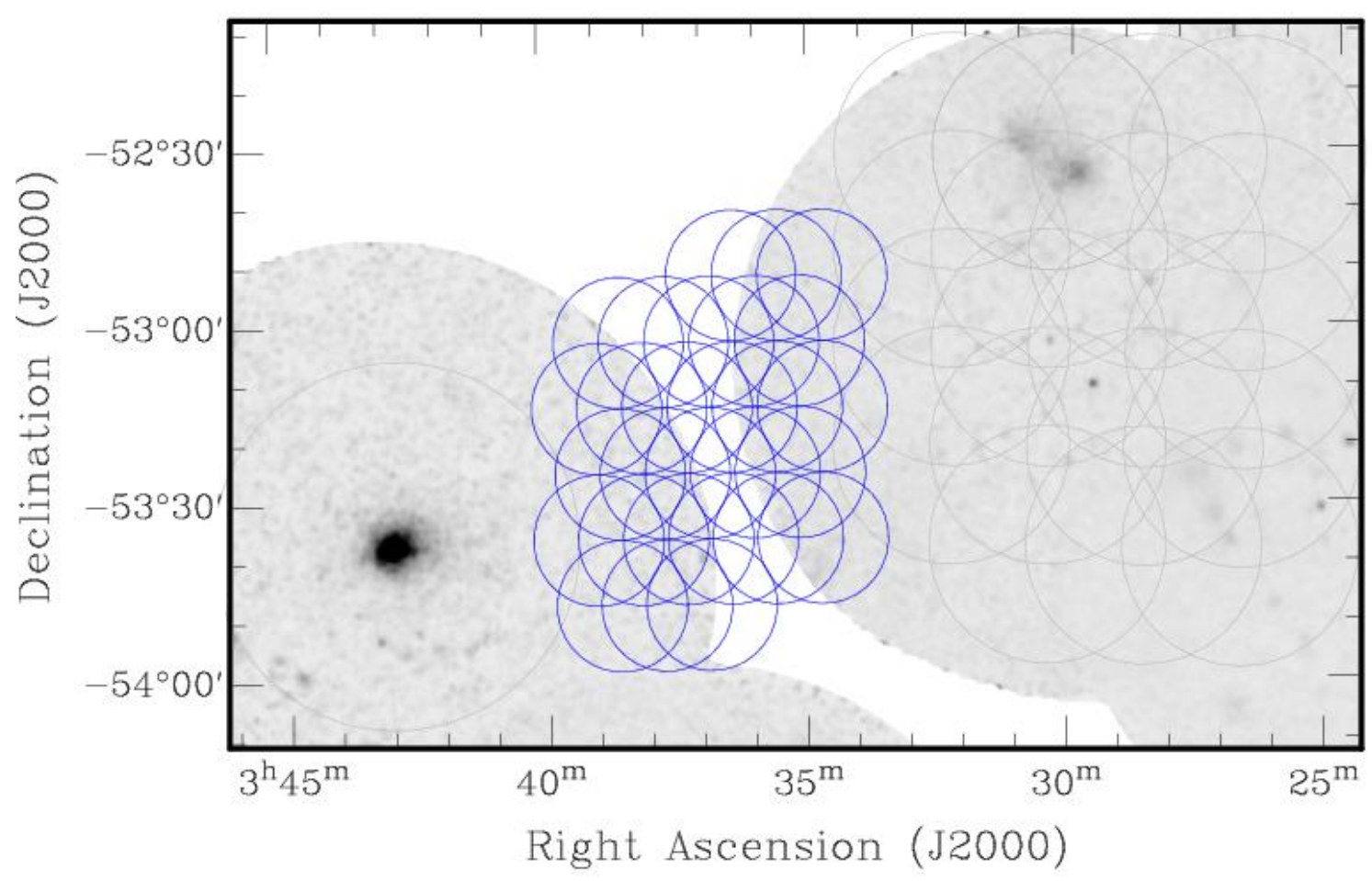

Figure 3.2: Our proposed mosaic for observation of Zone 1 filamentary region at $2.1 \mathrm{GHz}$. This region, located between A3158 (left) and the A3125/A3128 complex (right), required a total of 28 pointings (blue) to cover the previously unexplored region in the radio. Previous radio observations of the field are shown in grey on background ROSAT X-ray image. Note that previous radio observations were performed ad $1.4 \mathrm{GHz}$, resulting in a larger beam size than ours at $2.1 \mathrm{GHz}$, as explained in the text. 


\begin{tabular}{cccccrc}
\hline $\begin{array}{c}\text { Project } \\
\text { Code }\end{array}$ & Date(s) & $\begin{array}{c}\text { Observing } \\
\text { Time (min) }\end{array}$ & $\begin{array}{c}\text { Baseline } \\
\text { Config. }\end{array}$ & $\begin{array}{c}\text { Frequency } \\
(\mathrm{MHz})\end{array}$ & $\begin{array}{r}\text { Pointing } \\
\text { RA }\end{array}$ & $\begin{array}{l}\text { Centre } \\
\text { Dec (J2000) }\end{array}$ \\
\hline C959 & $08 / 08 / 2001$ & 87 & $1.5 \mathrm{~A}$ & $1384,2496,4800,8640$ & $03: 34: 07.2$ & $-39: 00: 3.2$ \\
& $07 / 08 / 2001$ & 91 & $1.5 \mathrm{~A}$ & $1384,2496,4800,8640$ & $03: 34: 07.2$ & $-39: 00: 3.2$ \\
& $03 / 08 / 2001$ & 100.3 & $1.5 \mathrm{~A}$ & $1384,2496,4800,8640$ & $03: 34: 07.2$ & $-39: 00: 3.2$ \\
& $16 / 06 / 2001$ & 181 & 375 & $1384,2496,4800,8640$ & $03: 34: 07.2$ & $-39: 00: 3.2$ \\
& $15 / 06 / 2001$ & 99 & 375 & $1384,2496,4800,8640$ & $03: 34: 07.2$ & $-39: 00: 3.2$ \\
& $14 / 06 / 2001$ & 127 & $750 \mathrm{~A}$ & $1384,2496,4800,8640$ & $03: 34: 07.2$ & $-39: 00: 3.2$ \\
& $13 / 06 / 2001$ & 142.7 & $750 \mathrm{~A}$ & $1384,2496,4800,8640$ & $03: 34: 07.2$ & $-39: 00: 3.2$ \\
& $21 / 05 / 2001$ & 150.7 & $6 \mathrm{~F}$ & $1384,2496,4800,8640$ & $03: 34: 07.2$ & $-39: 00: 3.2$ \\
& $20 / 05 / 2001$ & 29.7 & $6 \mathrm{~F}$ & $1384,2496,4800,8640$ & $03: 34: 07.2$ & $-39: 00: 3.2$ \\
\hline
\end{tabular}

Table 3.2: Previous observations of the centre of A3135, near the Zone 2 filament region, for which the project code was C959. PI for these observations was L Saripalli, and the source of interest was PKS J0334-3900. PKS B0332-403 was used as the secondary calibrator, with PKS B1934-638 the primary calibrator as usual. These data were recently published in Pratley et al. (2013). Additional pointings from projects P593 and P617 are shown on the map in Fig. 3.3, but these observations were too shallow to provide any useful data.

Such arguments are inevitably raised throughout astronomy whenever an attempt is made to infer universal truths from small, localised samples. All though we cannot fully escape from this - both major regions of interest are, after all, within the HRS - the repetition does offer some analytical peace-of-mind.

\subsubsection{Observations}

Observations were undertaken in three different sessions, simply due to the allocation of observing time by the ATCA time assignment committee in the 2012APR and 2012OCT semesters. These observations were all to be undertaken in continuum mode with $2 \mathrm{GHz}$ bandwidth and central frequency $2100 \mathrm{MHz}$. The baseline configurations were $6 \mathrm{D}$ in the first case, and $6 \mathrm{~B}$ and $1.5 \mathrm{D}$ in the second semester.

The first observing session granted to this project consisted of a single 12-hour block in June 2012 , during which it was desired to observe some portion of Zone 1 . Sacrificing $\sim 20 \%$ of the total for overheads, this left us with around 9.5 hours, insufficient to probe the entire field to the full depth desired $(0.05 \mathrm{mJy})$. So the options for observing were to:

(a) Use the available time to observe all 28 pointings in Zone 1, achieving whatever sensitivity is possible, then using future observing sessions to complete to full depth.

(b) Aim for full sensitivity in as many pointings as possible. As a sensitivity of $0.05 \mathrm{mJy}$ requires $\sim 1$ hr observation with $\mathrm{CABB}$, in the full session we could safely complete 9 of the 28 pointings.

(c) Try to reach a compromise between sky area observed and sensitivity. Covering more of the filament at reasonable sensitivity offers the potential for more robust statistics. If it is then found that greater depth is required, a portion of future observations can be spent on 'finishing off' this subregion. 


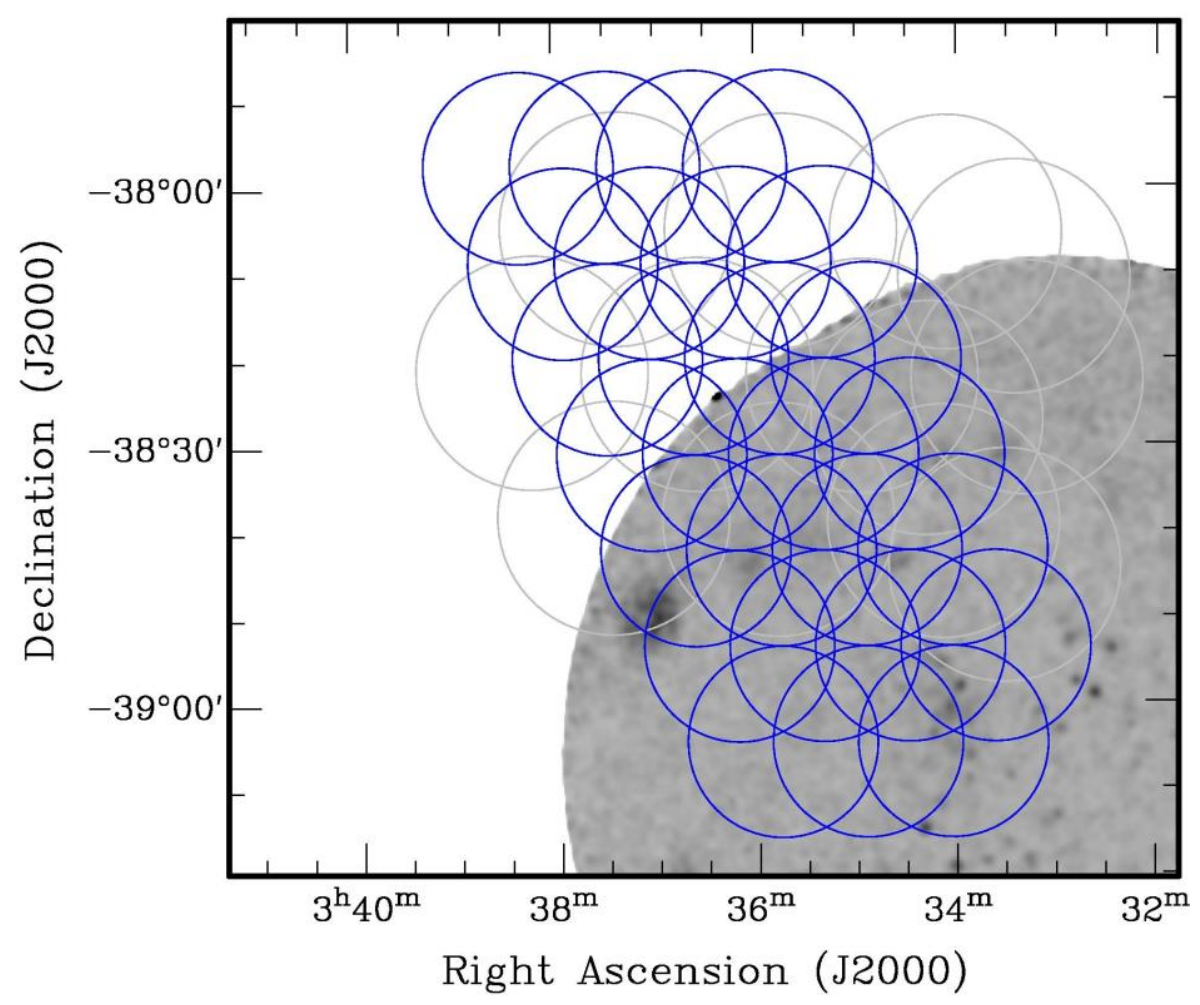

Figure 3.3: Our proposed mosaic for observation of Zone 2 at $2.1 \mathrm{GHz}$ with ATCA. The mosaic here consists of 27 pointings covering the region between A3135 (bottom) and A3145 (for which there were no previous ATCA or ROSAT observations). Previous radio observations of the field are shown in grey on background ROSAT X-ray image. These radio pointings were observed 17-21 September 2008 using Parkes at $1395 \mathrm{MHz}$, with 1025 channels and a bandwidth of $64 \mathrm{MHz}$ (ATOA project codes P593, P617). Unfortunately the pointings were each only 5.3, so would offer negligible sensitivity gain if added to the data for this project. Again, the pointing fields of view are larger compared to our proposed mosaic because of the lower central frequency. 
It was this final route that was eventually taken. The strategy, then, was to take our assigned 9.5 hours and divide that into some subset of the full Zone 1 mosaic, offering some intermediate sensitivity level. Following the inverse square rate of observing sensitivity (see Eqn. 1.1), a sensitivity level of half that desired, $0.1 \mathrm{mJy}$, should require $1 / \sqrt{2} \approx 0.7$ hours' observing for each pointing. This depth corresponds to a SFR of $0.21 \mathrm{M} \odot / \mathrm{yr}$, still a significant improvement on contemporary values. With the total observing session it should be possible to observe 14 pointings to this sensitivity - conveniently half of the Zone 1 field. To select this subregion, hereafter called Zone1(core), the other 14 fields were excised from the north, south and west of the total region. It was thought that the majority of filamentary sources would fall within the Zone1(core) subregion anyway.

Reduction of these initial observations (see below) revealed that sufficient sensitivity was indeed achieved, meaning that the second and third observing blocks could be devoted to the remainder of Zone 1 - hereafter Zone1(arm) - and to Zone 2. These observing blocks each consisted of two 12-hour sessions, making a total of around 60 hours assigned to this project and split between the two fields of interest. The baseline configurations of these sessions were $6 \mathrm{~B}$ and $1.5 \mathrm{D}$ respectively, and both used the $\mathrm{CABB}$ in $16 \mathrm{~cm}$ continuum mode.

For these later observations an on-the-fly approach was adopted. The strategy here was to first observe Zone1(arm) down to a comparable sensitivity to that already reached with Zone1(core). Because this field consisted of the same number of pointings as before, the same sensitivity could theoretically be achieved in the same time frame - that is, 12 hours. However the actual figure would fluctuate depending on properties such as weather and radio-frequency interference (RFI) that would cause us to throw away instrument time (for example, in a storm-stow situation) or raw data. Either case could limit the effective time used during observing. It is reasonable to assume that the allowance for standard observing overheads would remain the same.

Weather and RFI remained within manageable levels for the Dec 13 session, allowing allocation of the remaining three 12-hour slots to Zone 2, with the exception of $\sim 30$ minutes at the beginning of Dec 14. At that time Zone 2 had not quite risen above the $12^{\circ}$ elevation limit, so one further loop of Zone1(arm) was completed before continuing with Zone 2 .

Zone 2, which consisted of 27 mosaic fields, theoretically required similar length-of-observation to that of Zone 1 (28 fields) to achieve the same sensitivity. The success of Zone 1 observations on the preceding day meant that we actually had slightly more time than we needed. As it happened, though, this was just as well. The observations on 14 Dec 2012 and 01 and 02 Jan 2013 were plagued by rather more severe RFI than had previously been encountered. Further time was lost during the 01 and 02 Jan sessions as the Zone 2 mosaic fields passed through zenith.

Like most major radio telescopes currently in operation the individual elements of the ATCA are mounted in altitude-azimuth configuration. The effect of this is that as a source passes directly over the antenna (altitude $=90^{\circ}$ ) the mount must traverse the antenna through $180^{\circ}$ of azimuth instantly if it is to continue tracking the source without interruption. For a point source the problem is fairly straightforward, and delays typically last on the order of minutes. For a mosaic field the problem is compounded. If the centre of a mosaic field is directly above the antenna, this means we will have individual pointings straddling the zenith for an extended period, until the whole field has traversed zenith. With the integration time for each 


\begin{tabular}{ccccccccc}
\hline Date(s) & $\begin{array}{c}\text { Project } \\
\text { Code }\end{array}$ & $\begin{array}{c}\text { Target } \\
\text { Region }\end{array}$ & $\begin{array}{r}\text { Reference } \\
\text { RA }\end{array}$ & Pointing & Secondary & Pointings & ATCA & A \\
Calibrator & & Observing \\
Config. & Time (min) \\
\hline $23 / 06 / 2012$ & C2613 & Zone1(core) & $03: 39: 00.0$ & $-53: 15: 22.0$ & $0302-623$ & 14 & $6 \mathrm{D}$ & $\sim 576$ \\
$13 / 12 / 2012$ & C2767 & Zone1(arm) & $03: 34: 42.0$ & $-52: 53: 02.4$ & $0302-623$ & 14 & $6 \mathrm{~B}$ & $\sim 960$ \\
$14 / 12 / 2012$, & C2767 & Zone 2 & $03: 35: 45.6$ & $-37: 57: 50.4$ & $0438-436$ & 27 & $1.5 \mathrm{D}$ & $\sim 1152$ \\
$01-02 / 01 / 2013$ & & & & & & & & \\
\hline
\end{tabular}

Table 3.3: Summary of ATCA observations undertaken as part of this research project. All observations used the standard southern-sky flux/bandpass calibrator PKS B1934-638.

pointing only 10 seconds long (the default value, and the length chosen for these mosaics) we quickly find ourselves in arrears as the zenith-delay begins to exceed the integration time. The issue is a well-known one, but a common stumbling-block for inexperienced observers. As it was, a period of around 90 minutes each day was lost as the Zone 2 traversed zenith. After first encountering the problem on Jan 1, the following day the instrument was stowed for the duration of the traverse so as not to put any unnecessary strain on the hardware. To have begun observation with a few extra hours was a boon and this time was put to good use.

A summary of the observations undertaken within the scope of this project is given in Table 3.3. This provides the details of the three separate observing sessions; dates, project codes, targets observed and their corresponding secondary calibrators, baseline configurations and the approximate total observing time for each. All sessions used the ATCA in its $2.1 \mathrm{GHz}$ continuum observing mode with $2048 \times 1 \mathrm{MHz}$ channels.

\subsubsection{Reduction Process}

Reduction of all radio data made use of the MIRIAD suite (Sault et al. 1995), with standard procedures as outlined in the user guide ${ }^{1}$. For each observational field the pointings were inverted, cleaned and restored separately in Stokes I, before being combined via linear mosaic. The process was repeated in Stokes Q and U, to generate polarisation maps for each field.

\section{Calibration and Band Subdivision}

A slightly different approach was used between reduction of June 2012 observations and those from December 2012 - January 2013, as these reductions were undertaken at different times. The most obvious difference between, for example, the Zone1(core) data from June and the Zone1(arm) data from December was in the stability of the calibrators. The exceptionally stable gain and phase solutions across the full $12 \mathrm{hr}$ observing window allowed the June data to be calibrated and subsequently reduced as a single set, without the need to divide the observation into sub-bands. Here calibration followed the standard strategy as detailed in Fig. 3.4, with the simplification that Step 2 - copying bandpass solutions from the bandpass calibrator to the flux calibrator - was not necessary, as in this case they are one and the same.

\footnotetext{
${ }^{1}$ Available online, http://bima.astro.umd.edu/miriad/
} 


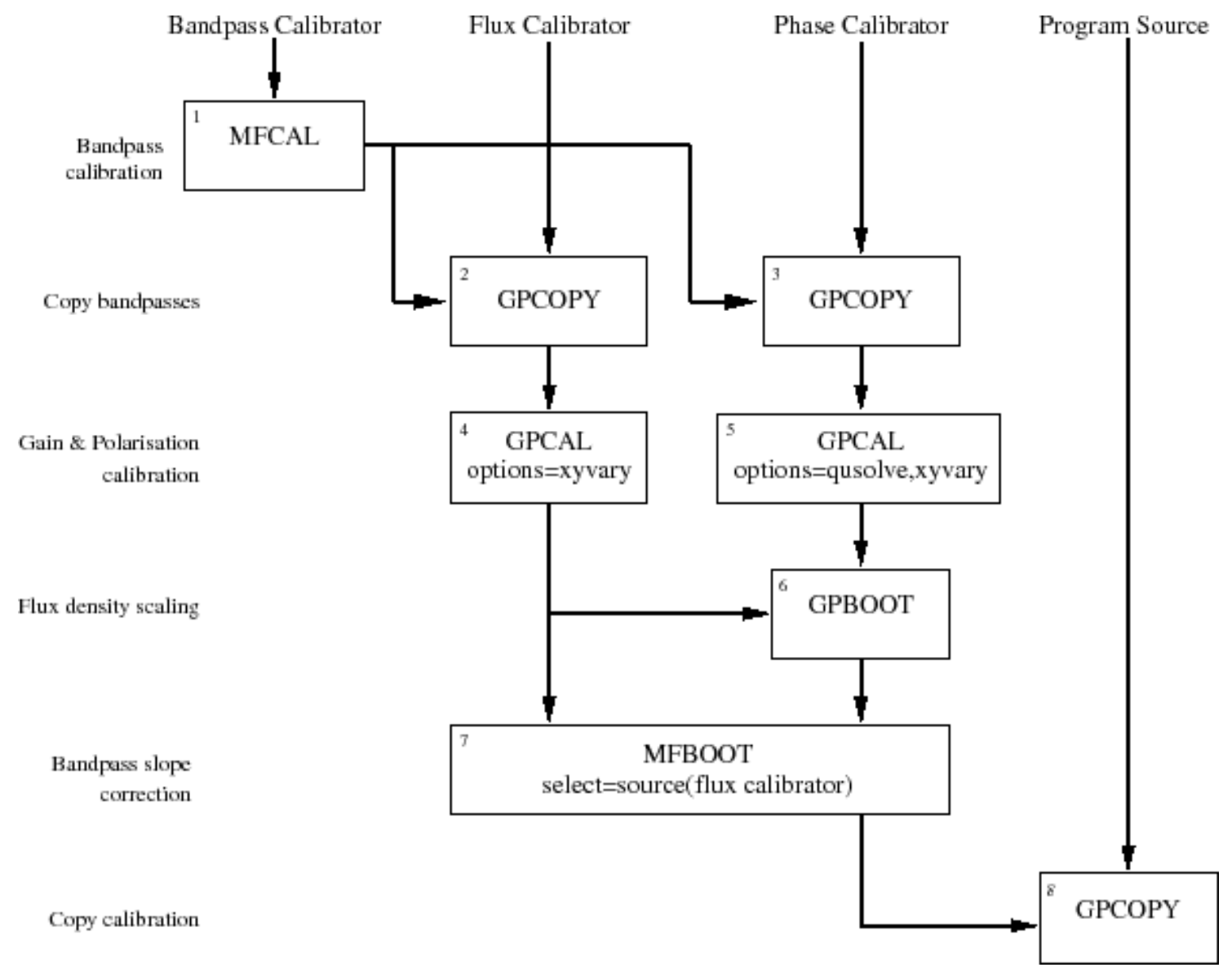

Figure 3.4: Calibration of observational data for Zones 1 and 2 followed standard procedures for CABB outlined in the MIRIAD user manual. There was a slight deviation from the above diagram in that for these observations PKS B1934-638 was used as both flux and bandpath calibrator, so the gpcopy in Step 2 was not necessary. 
A common practice adopted by astronomers working with CABB data is to use pgflag as an automated flagging utility on the various stokes parameters by selecting command = $\backslash \mathbf{b}$, rather than running pgflag in its standard, manual mode. This saves significant time in the flagging stage by cycling through all baselines and removing strong instances of RFI. It is generally felt among the ATCA community that this process offers a good compromise of thorough flagging, without being too 'heavy handed' and removing too much data.

This calibration strategy used here was generally the same as previously employed when reducing pre-CABB ATCA observations (see Section 1.4.1). Because of the $128 \mathrm{MHz}$ observing band of that instrument, significant drift of phases within the band were unlikely at cm-wavelengths. With the CABB-upgrade in 2009 variations in both gain and phase across the now- $2 \mathrm{GHz}$ band became far more pronounced. A strategy critical to dealing with CABB data is to not attempt a single calibration solution across the entire $2 \mathrm{GHz}$ band, but rather to split the data into several subbands to achieve a piece-wise calibration solution. Practically, this means selecting an nfbin value greater than one during the mfcal and gpcal calibration steps to allow a series of calibration solutions to be applied, better accounting for changing quantities. In theory the more frequency bins chosen, the more accurate will be the calibration solution, but a balance must be struck between the number of bins, and the number of unflagged data points in each bin. Here simple visual inspection of calibration data in the real/imaginary plane (for example, using uvplt) revealed that the difference between nfbin $=1$ and nfbin $=2$ was very pronounced. From nfbin $=2$ to nfbin $=4$ was still a marked improvement, while futher increase to the number of frequency bins did not yield a significant gain in quality of the calibration solutions.

Unusually the June 2012 observations yielded a very stable atmosphere, meaning that the full $2 \mathrm{GHz}$ data complement was actually able to be calibrated at once, as was the standard method for pre-CABB. For the remainder of the project we were forced to split the band into four $512 \mathrm{MHz}$ bands using uvsplit. The subbands would be kept separate, calibrated and reduced in parallel, before eventually being mosaiced back together to generate final images of our fields. Hereafter, description of reduction processes will refer to both June observations in a single $2 \mathrm{GHz}$ band centred at $2100 \mathrm{MHz}$ and Dec/Jan observations split into four bands with central frequencies of 1332, 1844, 2356 and $2868 \mathrm{MHz}$, unless stated otherwise.

With the bulk of reduction having to be repeated four times before the bands can be suitably recombined, the workload also increases proportionally. Mercifully, a large amount of the reduction process can be automated via the use of shell scripts. In this case repeating processes for different frequency bands is easily achieved by incorporating loops into the scripts. As these observations concerned mosaic fields with several pointings, some nesting of loops was necessary to cover all fields at all frequencies.

The full reduction process was broken into three main stages, and looped scripts written for each. These were:

(1) Flagging. Primarily automatic flagging with pgflag, with manual blflag flagging where necessary.

(2) Calibration. Because the majority of calibration process deals with the calibrators rather than the source fields directly, only the final gpcopy from secondary calibrator to source (stage 8 in Fig. 3.4) needed to be scripted up. 
(3) Imaging. This script, varied slightly to accommodate the different observing zones and field numbers, was the most complex. It comprised an inverting, a cleaning, and a restoring step for each field before the final stitching together of fields into a linear mosaic manually.

Details of the imaging stage will be discussed below, and full example scripts can be found in the appendices.

\section{Imaging - (i) Inverting}

When making test images from the earlier Zone1(core) observations it was noted that although the each $2.1 \mathrm{GHz}$ pointing could be covered by around 2250 Nyquist-sampled pixels ${ }^{2}$ (see Eqn. 3.4), when it came to stitching fields together into a linear mosaic, unsightly seams were still visible with image sizes up to 4096 pixels $^{2}$. Restricting the imsize variable to powers of two to maximise computational efficiency, this meant that expansive 9092 pixels $^{2}$ images were necessary, requiring remarkably long run times, especially at the cleaning stage; these reductions took place before the set up of the supercomputer SPOCK, as mentioned in Section 1.4.2.

Similar to the calculation of a telescope's field-of-view (Eqn. 3.1), the cell (pixel) size necessary to correctly sample the field is given by

$$
\theta_{\text {cell }} \leq \frac{k \lambda}{3 D}=\frac{k c\left[\mathrm{~ms}^{-1}\right]}{3 \nu[H z] D[m]}=\frac{1.22\left[\mathrm{~ms}^{-1}\right]}{\nu[M H z] \times 60[\mathrm{~m}]}
$$

Here $\mathrm{k}$ and $\lambda$ are as before, but $\mathrm{D}(\neq \mathrm{d})$ is the separation between elements of the interferometer, i.e. the maximum baseline distance.

Further trial and error with Zone1(arm) and Zone 2 observations revealed that these fields could be stitched together successfully with an imsize of 4096, though the aforementioned trade-off was that the split frequency bands resulted in a fourfold increase in the time required for each step of reduction. So the gain from dropping the field size by half (in two dimensions) was roughly cancelled out by bandsplitting. Of course, with access to SPOCK and the ability to run multiple reduction scripts at the same time, the actual time taken for reduction on comparable scales was greatly reduced.

\section{Imaging - (ii) Cleaning}

When working with images that cover a wide range of frequencies, as here, we must take into account that the flux density of sources is not constant, but will vary with frequency. The MIRIAD routine mfclean (multi-frequency clean) takes this into account, unlike clean, which is only suitable for imaging bands of narrow-enough frequency that flux values can be considered constant (for example, pre-CABB ATCA). The former, correct algorithm was used throughout this project, except for dry-run testing. A detailed description of the routine can be found in Sault and Wieringa (1994), suffice to say here that mfclean requires extra 
input in the form of a 'spectral dirty beam', which can be optionally created as a by-product during the invert stage. Other than that and lacking a few of the non-essential features of clean, it performs the same role - at least at the macroscopic level.

mfclean could not be successfully run without first defining a subregion to apply the algorithm to. So after performing a test reduction of each field, region files with clean boxes around prominent sources were generated for each using cgcurs. Each field had 10-20 nonspurious sources that were boxed in this way. A side-effect of this is that the final fluxes for clean-boxed sources ought to be more accurate.

Different numbers of iterations of mfclean were run, from around a thousand to more than a million in some cases. Mostly optimal cleaning was achieved well below $10^{5}$ iterations, so this was used as an upper limit, with the actual cleaning depth for each field defined by the noise level with the cutoff variable. Unsurprisingly there was some variation of the noise level between fields. For the most part the $3 \sigma$ noise was about $1.0 \times 10^{-4} \mathrm{Jy}$, so this was used as the default cutoff. Following this, those fields with differing noise levels were re-cleaned to appropriate depths.

\section{Imaging - (iii) Restoration and Mosaicing}

Finally, the fields were restored and stitched together using linmos. Where images of different frequencies were joined together to make the final mosaic, their resolutions were matched by restoring using the map and clean component images of each frequency, with the beam file of the lowest frequency $(1332 \mathrm{MHz})$. Doing this in the restor step meant that we didn't need to deal with multiple map, beam, and clean files for each frequency with slightly different resolutions. The final radio mosaics for Zones 1 and 2 are presented in Figures 3.5 and 3.6. The $(3 \sigma)$ sensitivities of these maps are 0.10 and 0.20 mJy respectively, corresponding to radio powers of $\log \mathrm{P}_{2.1}=20.9$ and 21.2. These depths put the observations firmly in the star-formation-dominated regime, particularly in the case of Zone1 (recall the changeover point between AGN- and SF-dominated populations occurs at $\log \mathrm{P}_{1.4} \approx 22.8$ (Miller and Owen 2003)).

A cursory glance at the two mosaics in Figs. 3.5 and 3.6 reveals a marked difference in image quality, due primarily to radio artifacts associated with bright sources in Zone 2. Ideally this would not have been the case - it would have been desirable to have two fields with comparably deep radio images. However, comparison of the two filamentary zones was not the primary focus of this project. The central aim was to contrast the radio population of a filament with that of a cluster. Although two strong catalogues would provide allow for more robust conclusions, the majority of analysis could be adequately performed with only one. This will be alluded to again when it resurfaces later in this chapter.

\subsubsection{Image Preparation}

Before it was possible to perform any analysis of the radio populations in Zones 1 and 2, some obvious preliminary steps were necessary. First the full field maps of Figs. 3.5 and 3.6 had to be trimmed to remove the noise-dominated edges of each field. 


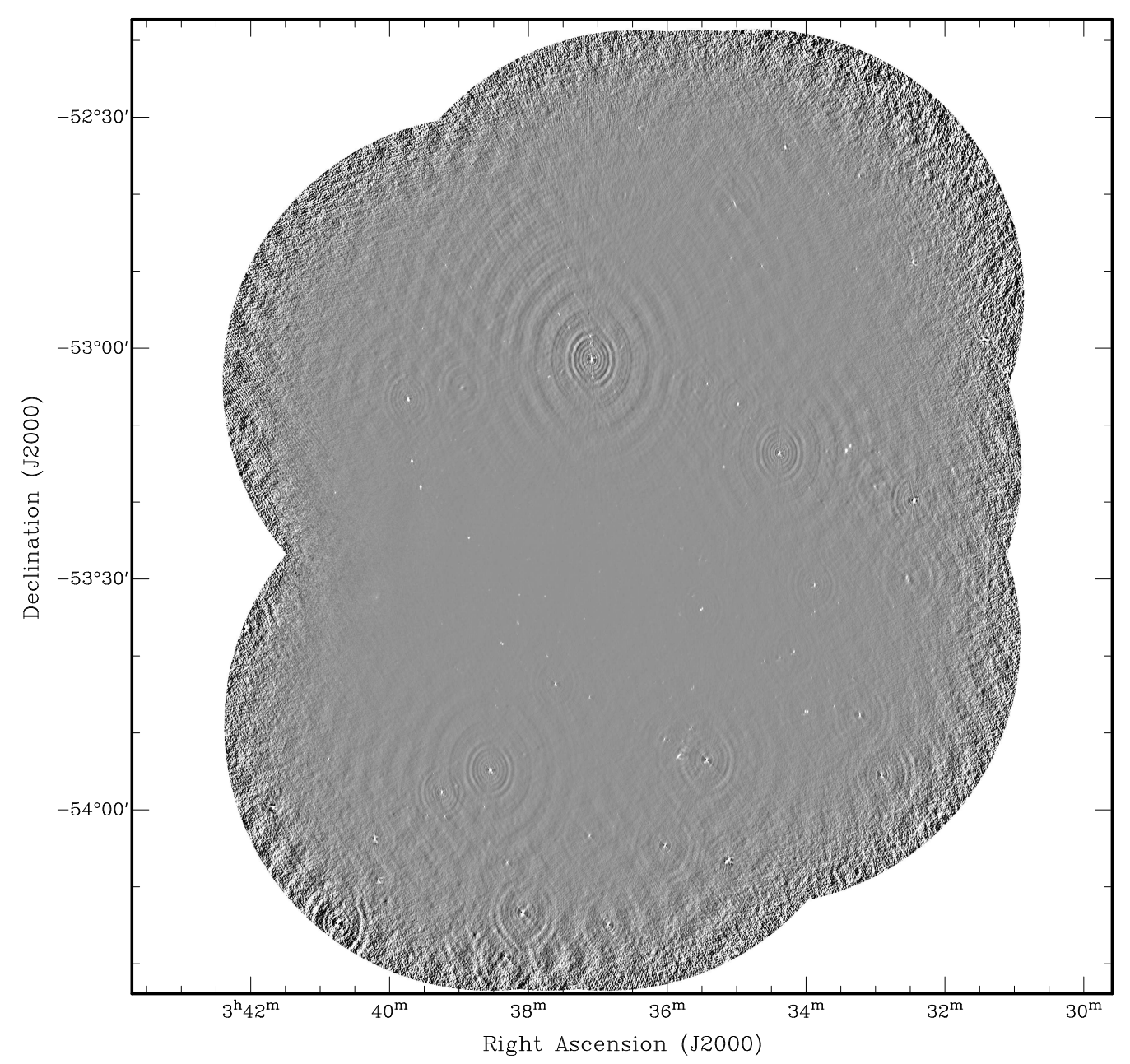

Figure 3.5: Final mosaiced radio map for Zone 1 filament region. This mosaic consists of 28 ATCA pointings at $16 \mathrm{~cm}(\mathrm{CABB})$, and has a $3 \sigma$ sensitivity of $0.10 \mathrm{mJy}$, corresponding to a radio power of $\log \mathrm{P}_{2.1}=20.9$. 


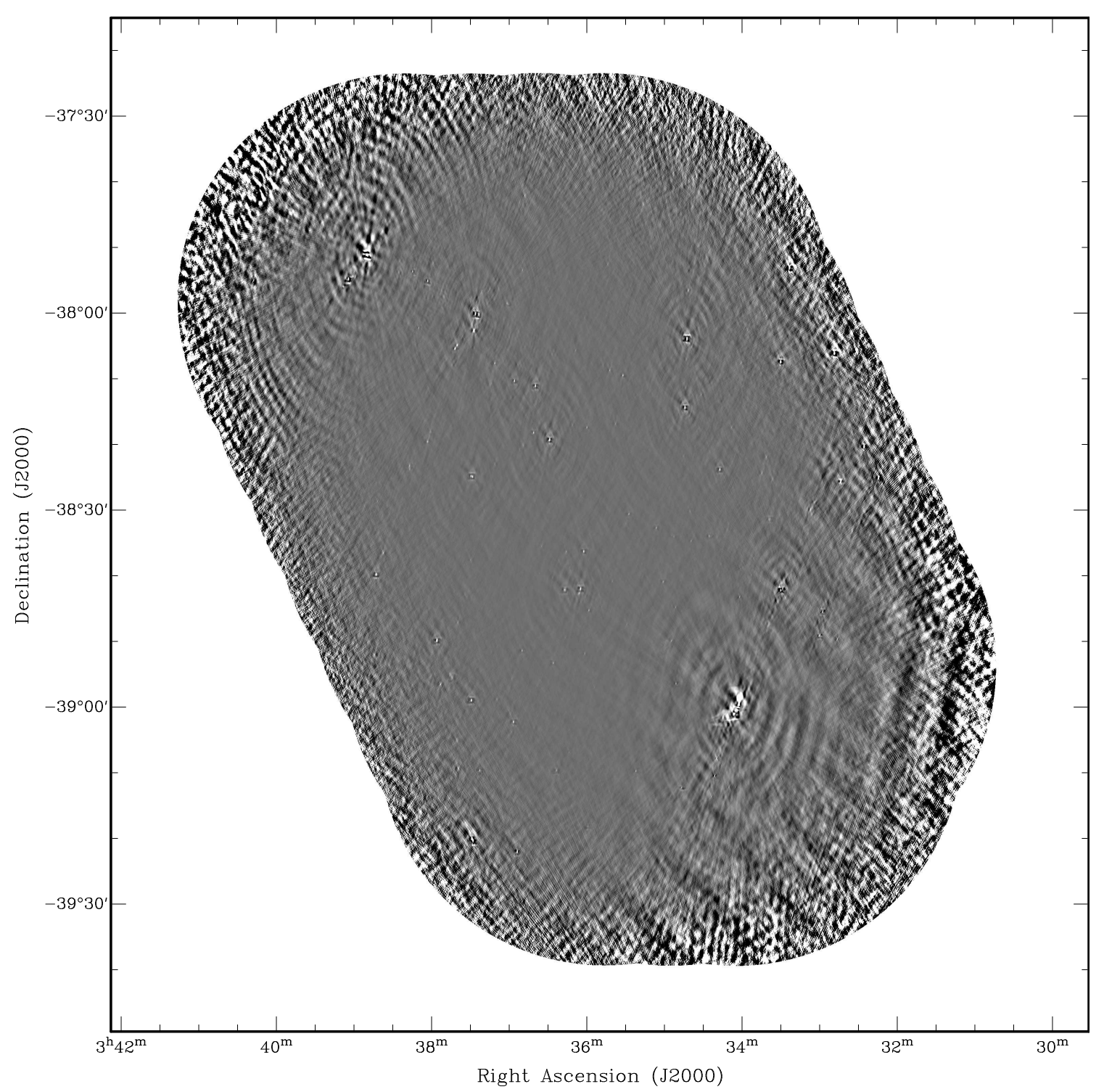

Figure 3.6: Final mosaiced radio map for Zone 2 filament region. This mosaic consists of 27 ATCA pointings at $16 \mathrm{~cm}(\mathrm{CABB})$, and has a $3 \sigma$ sensitivity of $0.20 \mathrm{mJy}$, corresponding to a radio power of $\log \mathrm{P}_{2.1}=21.2$. 
In any radio image the sensitivity is not uniform across the beam, but rather decreases uniformly with radius from the centre. As a result the usable portion is rather smaller than the full image. When we combine images in a linear mosaic the associated sensitivity map also becomes the combination of sensitivity maps from the individual pointings. Normally we would use the MIRIAD task mossen to generate this map, from which we could trim our radio image to some minimum fractional sensitivity - usually $80 \%$. For unknown reasons, attempts at this method were unsuccessful; mossen would simply output the sensitivity profile of the first pointing in the mosaic, ignoring the others. This was better than nothing, as it allowed the rest of the sensitivity map to be built up manually using notional copies of the original, and then the mosaic trimmed to where the outline of this shape would be using cgcurs.

Following this, catalogues of the radio populations had to be generated. For fields containing only a few sources this is achievable by hand but beyond about $\mathrm{n}=20$ the task becomes increasingly cumbersome and inaccurate as we rely on human judgement to discern between source and artifact.

\subsubsection{Source Detection}

Source detection for the fields was done using the Duchamp source detection algorithm (Whiting 2012). While that software was primarily intended for source detection in spectral-line data cubes, it also functions as a fairly effective detector of radio-continuum sources.

Duchamp allows for detection with a variety of different input and output options. For this project it was primarily used for source finding with an $\mathrm{n} \sigma$ cutoff - that is, considering only those sources detected above some multiple of the noise level of the field, which is independently calculated by Duchamp. A useful feature of the algorithm is that it allows region growing to a lower flux level than used for the initial detection. For Zone 2 a catalogue was generated for sources with flux above $3 \sigma$, with growth allowed down to $2.5 \sigma$ for each source, but in Zone 1 Duchamp had some trouble from artifacts with this cutoff level, so it was increased to $6 \sigma$, with growth to $5 \sigma$. Increasing the detection threshold from $3 \sigma$ to $6 \sigma$ reduced the source counts by $\sim 75 \%$ at all flux levels. A small number of spurious detections were also avoided by limiting classification as a true source to those of 8 pixels or more in extent.

The difference in the image quality achieved for the two Zones (refer back to Figs. 3.5 and 3.6) became evident in the Duchamp-generated catalogues. Although the fields were approximately the same size (see below) the catalogue for Zone 1 contained 332 radio sources, while the Zone 2 catalogue had only 100. These correspond to the rms noise levels of the fields, being 0.033 and $0.068 \mathrm{mJy}$ (see 3.1.3). In both cases these counts were incomplete. Inevitably some instances of noise were erroneously detected as sources, whilst some actual sources were missed because of their location near strong artifacts, despite themselves being above the detection threshold relative to the overall noise level of the field. Even for the relatively small fields observed here it was not really feasible to go through the catalogues by eye, manually removing false-positives, and rectifying false-negatives. This will be an increasing problem for next generation radio telescopes, meaning that the move from manual source detection to effective, fully automated detection algorithms will be crucial. 


\section{Field Sizes}

Another task necessary for all basic analysis of the intensity images was to accurately measure the sizes of the two observed fields over which Duchamp was applied. This was done by dividing each field into a number of simple polygons, and the area calculated via the trigonometric formula for the angular separation of two points on a sphere along a geodesic:

$$
b=\cos ^{-1}\left[\sin \delta_{1} \sin \delta_{2}+\cos \delta_{1} \cos \delta_{2} \cos \left(\alpha_{1}-\alpha_{2}\right)\right]
$$

where $\alpha_{i}$ and $\delta_{i}$ are the right ascension and declination of each point (being careful to convert each to appropriate units). From this treatment, it was found that Zone 1 covered an area on the sky of 1.1 square degrees, while Zone 2 was marginally smaller at 0.99 square degrees.

\section{Flux Scaling}

With our radio source catalogues generated and our regions defined it was possible to continue with an analysis of the source counts in each field, as had earlier been done for the Fornax Beta Field catalogue (see Section 2.2.1). As with that data the integrated fluxes of these radio sources, detected at $\nu=2.1 \mathrm{GHz}$, had to be corrected to $\nu=1.4 \mathrm{GHz}$ in order to properly compare with the results of the ATESP Survey from (Prandoni et al. 2001). This was again done by assuming a straight spectral index of 0.7 for all sources, so that correction is simply achieved via equation 2.2 with the same numbers as before. 


\section{Chapter 4}

\section{Analysis of Zone 1 and Zone 2 Filaments}

\subsection{Radio Intensity Properties of Zones 1 and 2}

\subsubsection{Source Counts}

For each Zone the source count profile was first generated for the whole population. Additionally, profiles were drawn from notional filament- and non-filament subregions. For Zone 1 this region selection was based on the prior designation by Fleenor (2006, also see Fig. 3.1). For Zone 2 the situation was more simple, as there are only two clusters nearby. A straight channel of width 2 Abell radii was selected running between cluster centres (as defined in Abell et al. 1989). From these defined subregions, the Duchamp catalogue sources could be divided into the same categories based on their coordinate information. The areas of the two filamentary subregions were 0.40 and 0.70 square degrees for Zone 1 and 2, respectively. The comparative source count profiles are presented in Figs 4.1 and 4.2 and Figure 4.3 comprises the comparative source counts from Zones 1 and 2 as a combined population.

What is unusual about these source counts is not the overall shape, which is as expected (we do see a significant drop in the counts at the low end of Zone 1 due to incompleteness of the sample. A similar effect is evident in Zone 2, but it is not so prominent), but the fact that the plots for both Zones imply that the number of sources hosting radio emission at all powers is lower in the filament than outside of it.

The robustness of the filament/non-filament source count profiles is questionable. It seems likely that the low counts and small areas of these subregions are affecting the profiles' quality. Because of this, further analysis will only consider the catalogues of each field as a whole, without dividing them into filament/non-filament subregions.

In Fig 4.4 the overall source count profiles for Zones 1 and 2 are compared to the earlier profile from the Fornax Beta Field, and to the ATESP Survey benchmark from (Prandoni et al. 2001). First, consider the profile for Zone 2. This appears to closely adhere to the ATESP line, though with some scatter due to the small sample size used. At around $3 \mathrm{mJy}$ 


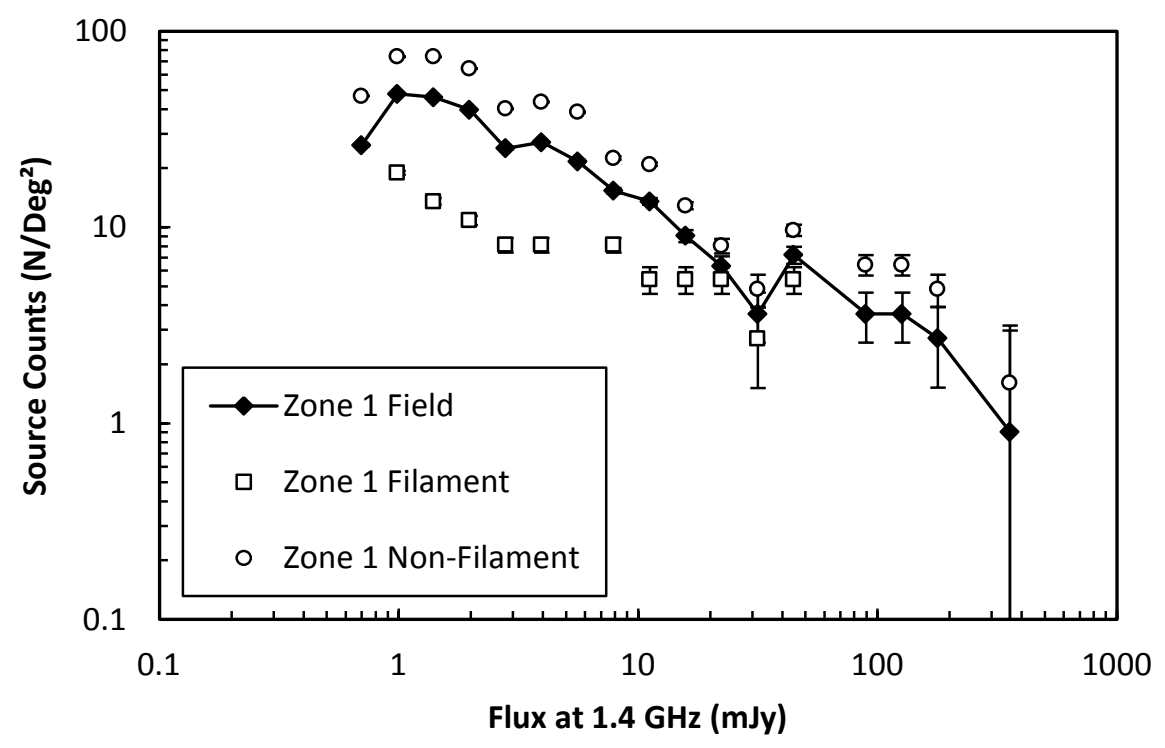

Figure 4.1: Zone 1 source count profile, comparing the full catalogue with filament and nonfilament subpopulations. The apparent excess in source counts in the non-filament region, and deficit within the filamentary population, is an unexpected result. Errors are $2 \sigma$ Poissonian.

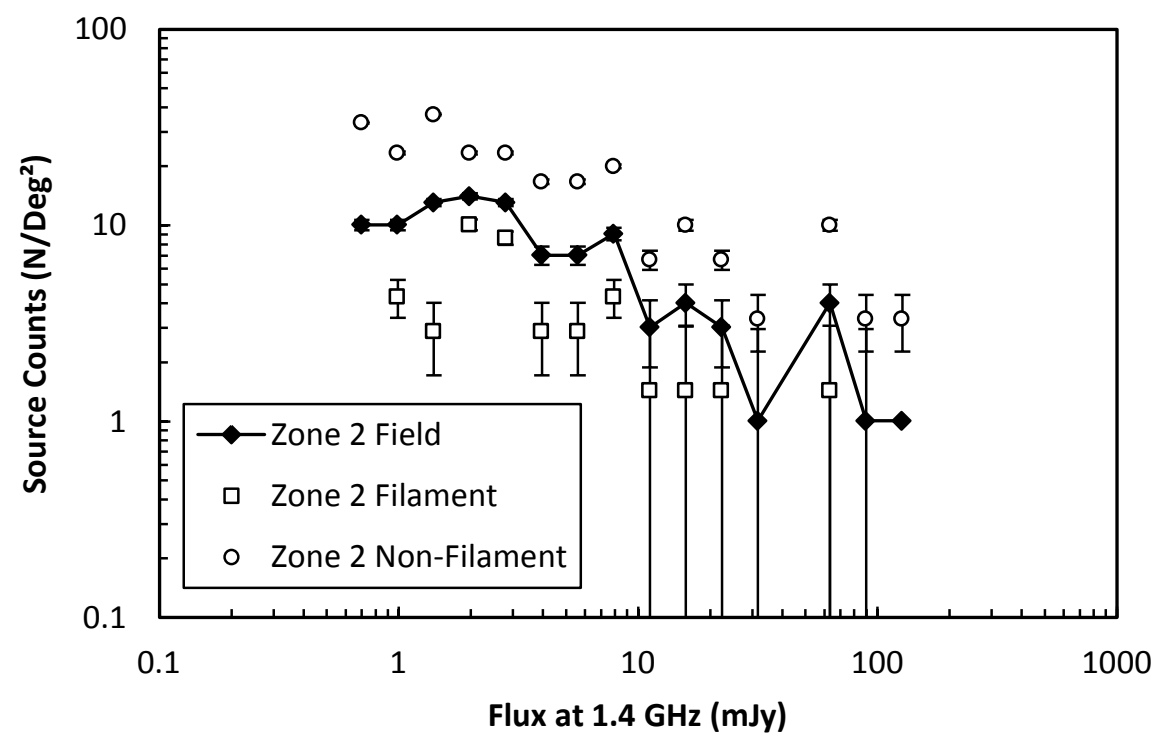

Figure 4.2: Zone 2 source count profile. Again here we see a source count excess and deficit in the non-filament and filament populations respectively, contrary to expectations. Errors are $2 \sigma$ Poissonian. 


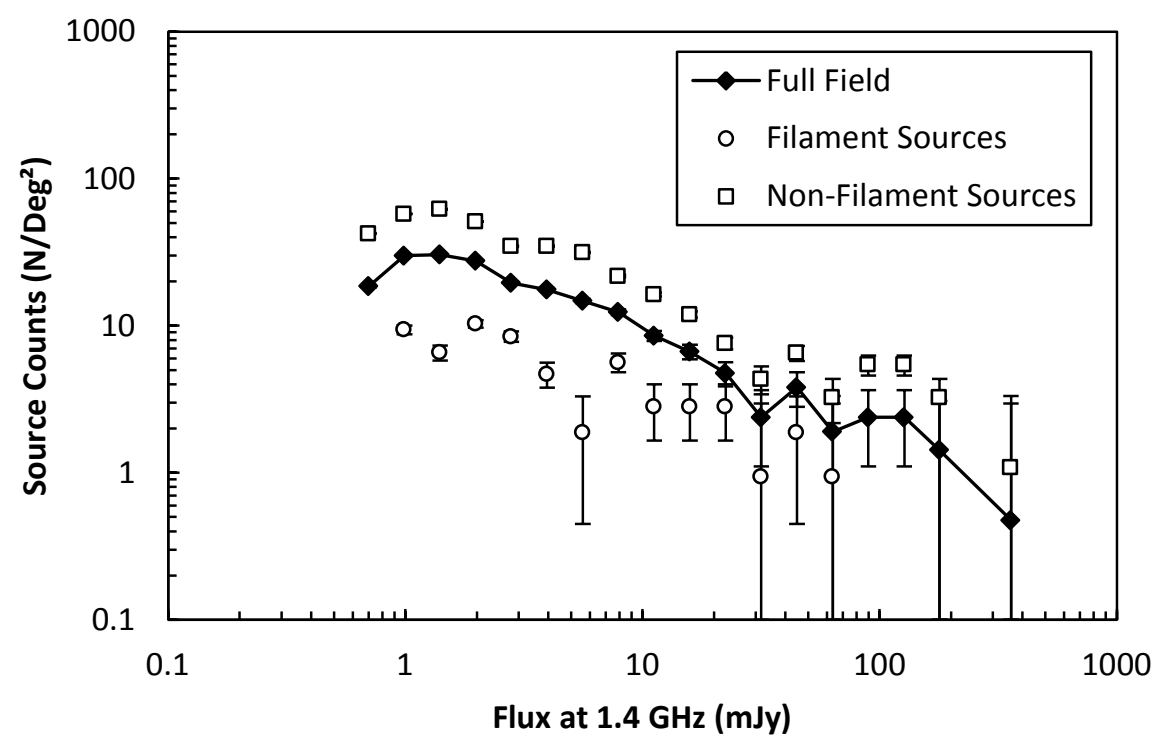

Figure 4.3: Source count profiles of Zones 1 and 2 were averaged together to arrive at a mean profile allowing for a larger population over an area approximately twice the size. Of course, this assumes that the two regions should exhibit the same general properties, both being filamentary regions spanning similar distances between rich clusters in the HRS. As is discussed in the text, this assumption isn't necessarily valid. Errors are $2 \sigma$ Poissonian.

we lose completeness in the catalogue, and we see it fall below the line of Prandoni et al. at the bottom end.

In contrast with this is the profile of Zone 1, which appears to exhibit a marked excess of radio sources at all flux levels relative to the other fields, down to the completeness limit of $\mathrm{S} \approx 1.0 \mathrm{mJy}$. This flux limit corresponds to radio power $\log \mathrm{P}_{1.4}=21.9$ at the mean redshift of the HRS, well within the SF-dominated regime, implying that we would be very unlikely to see powerful AGNs in these regions. There remains the possibility that false detection by Duchamp is affecting the source counts for Zone 1, resulting in an apparent excess when there should not be one. With Duchamp initially set do detect sources at the $3 \sigma$ level, it was found that the program picked up an excess some ten times above the expected profile. This was brought down to a factor of $\sim 3$ above expected by limiting the cutoff to $6 \sigma$. Taking into account a $2 \sigma$ Poissonian uncertainty estimate associated with possible binning error, the source counts for Zone 1 still appear to be significantly above the expected values for flux levels $1 \mathrm{mJy} \leq \mathrm{S}_{1.4} \leq 200 \mathrm{mJy}$.

Another possible cause for the disparity between Zones 1 and 2 is a miscalculation of the area of the fields. However, as the source count density scales linearly with area, the profile is not terribly sensitive to small errors - especially when viewed on a log scale. Even if the areas of both Zones were off by $100 \%$ - that is, if we doubled the area of Zone 1 and halved Zone 2 , the source counts would still show significant disagreement, except in the regime above $\sim 50$ mJy. So ought the two filamentary regions show similar source count profiles? Not necessarily. It is likely that the variance between the regions can be adequately explained by 


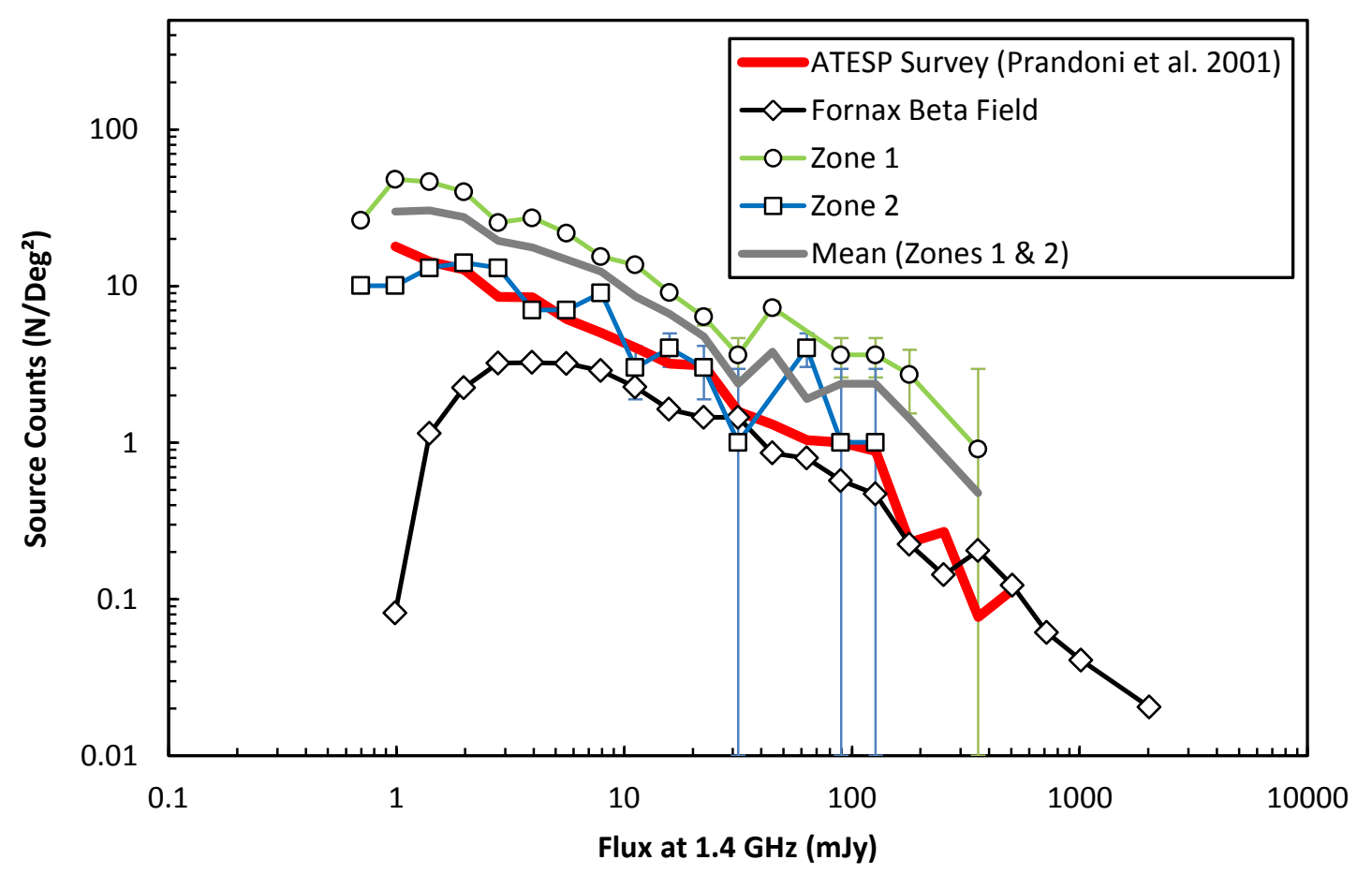

Figure 4.4: Integral source counts for Zone 1 and Zone 2 radio populations added to earlier plot of Fornax Field and ATESP Survey results. Also in grey is the filament average source count profile drawn from the combination of the two zones. Errors are $2 \sigma$ Poissonian, with the exception of Zone 1, which additionally has negative errors drawn from the differences between these counts and those from a second Duchamp catalogue with a $10 \sigma$ cutoff, as discussed in the text. Errors for Fornax Field population as in Fig. 2.8, so have been omitted here for clarity.

cosmic variance, emphasised by the small dimensions of the fields (compared with the survey sizes of the other studies plotted in Fig. 4.4). This issue will be further addressed in Chapter 5.

\subsubsection{RLF}

Following the source count analysis of Zones 1 and 2 in the previous section it was desirous to investigate the Radio Luminosity properties of both fields, as had earlier been done for the Fornax Beta Field (see Section 2.2.2). The treatment here was exactly the same as the method described in that section and approximate distances, redshifts, etc, were taken again from those attributed to the A3125/A3128/A3158 complex in (Johnston-Hollitt et al. 2008).

As explained in 2.2.2, both univariate and bivariate radio luminosity functions (RLFs) rely on relating our radio population data for a field to its corresponding optical catalogue. Because we are here working with faint (optically and in the radio) sources to begin with, the quality of 
any analysis will be limited by the extent to which our radio and optical catalogues correlate. This was also the case for the Fornax Field catalogue. The difference here is that each of our fields is significantly smaller than the sky area covered in that survey. The areas of Zones 1 and 2 were $1.1 \mathrm{deg}^{2}$ and $0.99 \mathrm{deg}^{2}$ respectively (see Section 4.1.1), while the Fornax Beta Field covered some 49 square degrees. It is not surprising that the number of optical/radio correlations derived from NED, along with the SuperCOSMOS catalogue via the TOPCAT source-matching package ${ }^{1}$, were rather fewer than those for the Fornax Field population. Zone 1 yielded 21 radio sources with associated optical data. Because of its lower overall count only 4 matches were detected in Zone 2. This is obviously insufficient for any useful RLF analysis, so that field was discarded for the remainder of this section of the project.

For RLF analysis again it was desirous to check whether the full radio population of the region could be suitably described by considering only those sources with optical counterparts. For the Fornax Beta Field catalogue, we saw that the two populations did indeed display very similar distributions for the fluxes of sources. This was mainly due to the large number of optical matches found (83, around $6.4 \%$ of the total population), allowing for a fairly smooth distribution within the subpopulation. In Zone 1, for which the corresponding histograms are presented in Fig. 4.5, we see that the correlation between the distributions of master catalogue and optically-matched subpopulation is less clear. Here we were working with much fewer optical matches; 21 out of 332 , or around $6.3 \%$. The similar optical-radio/radio ratio here is another indicator that the source counts generated in Section 4.1.1 are in an appropriate regime. To boost our sample size slightly a further 5 radio-optical sources for Zone 1 were drawn from an older Duchamp-catalogue with $3 \sigma$ cutoff and checked against the map (Fig. 3.5). One of these, corresponding to a very optically bright source $\left(\mathrm{b}_{J}=\right.$ 13.85), was declared spurious and removed. The remaining 4 were added to the radio-optical catalogue, giving 25 in total. The low number of sources makes it difficult to say whether the two populations exhibit similar flux distributions. In the figure there is no evidence to suggest the contrary, and we would not expect that to be the case. However, evidence for strong correlation is not robust. Of course, without performing additional observations there is little to be done about that. We shall therefore continue with the data available, but must keep in mind that any conclusions drawn thereof will be rather less solid than those regarding the Fornax Field population.

The univariate radio luminosity function for Zone 1, presented in Figure 4.6, shows a similar trend to that of the Fornax Beta Field (Fig. 2.10). This general shape is to be expected for a cumulative probability distribution. At a given optical magnitude cutoff (higher magnitude $\approx$ lower brightness) the cumulative probability must monotonically increase as we move to lower radio flux values. The likelihood of detecting any radio sources within the optical population generally increases as we limit ourselves to brighter (lower) optical magnitudes.

Close inspection of the plot in Fig. 4.6 reveals that we see some evidence of high-powered (AGN) radio emission, but this does not appear as we move to brighter optical magnitudes. What this means is that these AGNs are optically weak, possibly background, sources. If this was the case, it would imply that there are no powerful AGNs to be found within the filament - i.e. they are limited to high-density environments. Unfortunately the three sources in question did not have redshift values in the catalogues from which they were drawn, so this hypothesis cannot be confirmed.

\footnotetext{
${ }^{1}$ Tool for OPerations on Catalogues And Tables; http://www.star.bris.ac.uk/ mbt/topcat/
} 


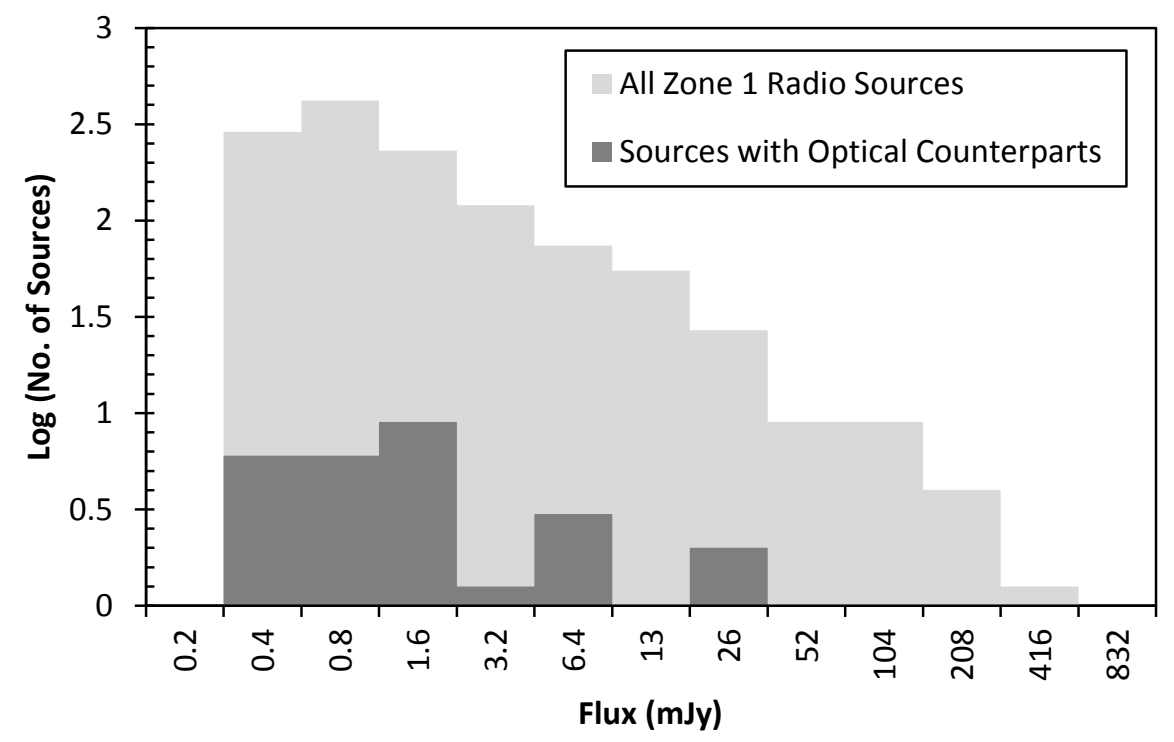

Figure 4.5: Comparison of the fluxes of sources in the Zone 1 catalogue, from Duchamp. The full radio catalogue distribution (light grey) is compared with that of radio sources with associated optical magnitudes, from NED and SuperCOSMOS online catalogues (dark grey). Bins with a single source have been artificially boosted to a little over 0 (as $\log [1]=0)$ to distinguish them from bins with no sources. The counts in the four rightmost bins for the optical population are 1, 3, 0 and 2, so it is unlikely the apparent peak at flux $\sim 26$ significant to within Poissonian uncertainty. 


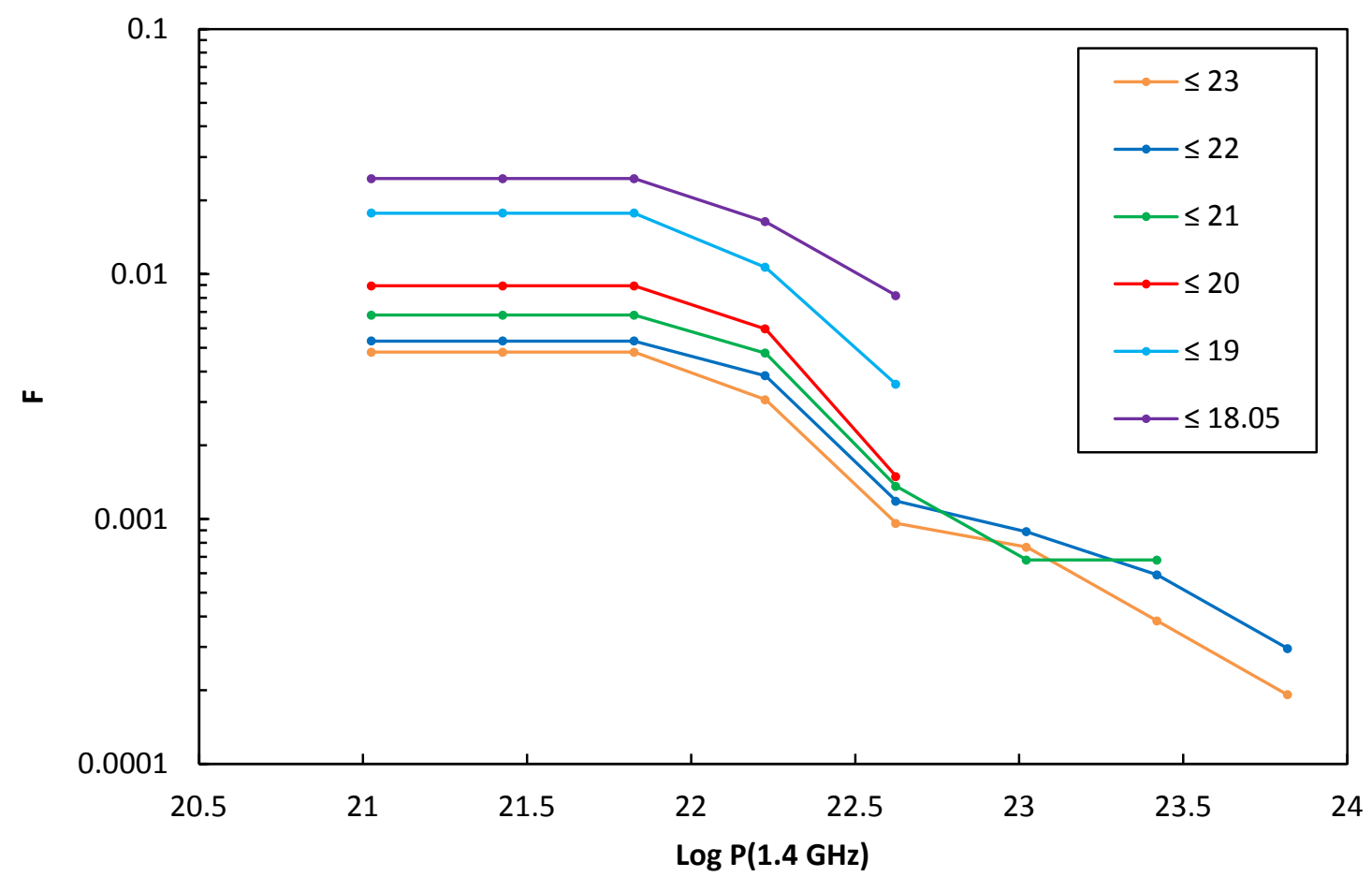

Figure 4.6: Integral radio luminosity functions for the radio/optical population of Zone 1, with different optical magnitude $\left(\mathrm{b}_{J}\right)$ cutoffs. 


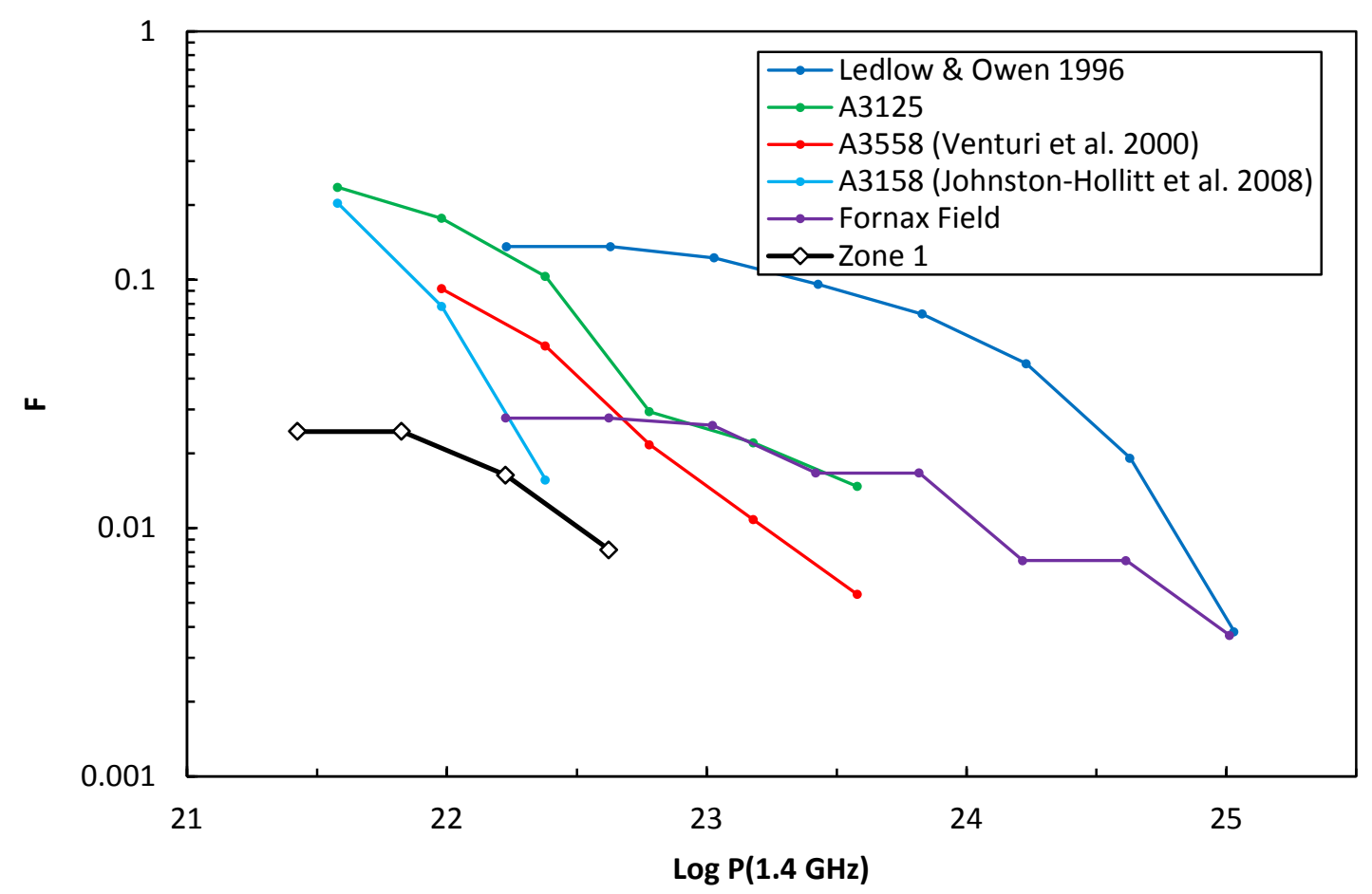

Figure 4.7: RLF for Zone 1 compared with a range of functions for different radio/optical populations. In order to compare our RLF with the canonical example from Ledlow and Owen (1996) we were limited to adopting an optical magnitude cutoff of $b_{J}=18.05$, which unfortunately forces us to exclude the bulk of our population. Being a filamentary region, this is understandable made up primarily of radio-weak, and optically-faint sources. The resulting RLF is drawn from only three sources. 


\begin{tabular}{cccccc}
\hline$\Delta \log \mathrm{P}_{1.4}$ & $\Delta \mathrm{S}_{1.4}$ & Fractional BRLF & \multicolumn{3}{c}{ Integral BRLF } \\
\hline $21.03-21.43$ & $0.13-0.33$ & $0 / 123$ & 0 & $3 / 123$ & 0.02439 \\
$21.43-21.83$ & $0.33-0.82$ & $1 / 123$ & 0.00816 & $3 / 123$ & 0.02439 \\
$21.83-22.23$ & $0.82-2.06$ & $1 / 123$ & 0.00816 & $2 / 123$ & 0.01626 \\
$22.23-22.62$ & $2.06-5.15$ & $1 / 123$ & 0.00816 & $1 / 123$ & 0.00816 \\
$22.62-23.02$ & $5.15-12.9$ & $0 / 123$ & 0 & $0 / 123$ & 0 \\
\hline
\end{tabular}

Table 4.1: Details of the bivariate radio luminosity function generated from the Zone 1 population with an optical magnitude cutoff of $\mathrm{b}_{J}=18.05$.

The bivariate RLF for Zone 1 (with optical magnitude cutoff of $b_{J}=18.05$, details in Table 4.1) is compared with RLFs from other studies in Fig. 4.7, for which the treatment was the same as in Section 2.2.2. In the plot we see no evidence for increased star formation in filaments and no evidence of AGN, as the RLF for Zone 1 included no sources above the $\mathrm{SF} / \mathrm{AGN}$ crossover power of $\log \mathrm{P}_{1.4} \approx 22.8$. AGN are found in dense regions in general but in clusters in both the HRS and Shapley which are in the late stages of a merger (postcore passage) their numbers are suppressed (Venturi et al. 2000; Johnston-Hollitt et al. 2008, Johnston-Hollitt \& Grimwood in prep.), in filaments in the HRS we see no evidence for AGN at all.

A shortcoming of the bivariate RLF plots in Figs. 2.11 and 4.7 is that in order to compare the RLF's for Fornax Field and Zone 1 populations to those from other studies, we are forced into a regime where $\mathrm{n}$ is extremely low. To generate the RLF's we used a total of fifteen sources for Fornax Field, and three for Zone 1, all that was available once our catalogues were limited to an optical magnitude cutoff of 18.05 (again, this is to properly compare our RLF with that of Ledlow and Owen 1996). In both fields we expect the vast majority of sources to be radio weak, and probably optically faint. A useful plot to complement that in Figure 4.7 is presented in Fig. 4.8. In this plot the RLF lines for both fields corresponding to an optical cutoff of 22 are shown. This gives us 73 sources for Fornax Field and 18 for Zone 1. Although using this optical cutoff means we can no longer compare with other surveys, it allows us to better compare the two main fields used in this study for RLF analysis. Full details of the RLFs in the plot are given in Tables 4.2 and 4.3 .

The RLF for Zone 1 in Fig. 4.8 seems to exhibit an increase in galaxies below $\log \mathrm{P}_{1.4} \approx 22$ (within the SF-dominated regime defined by Miller and Owen 2002), compared to the Fornax field which here is serving as a control. This is in contrast to the RLF above $\log \mathrm{P}_{1.4} \approx$ 22.5, where Zone 1 shows a deficit relative to the Fornax Field population. Over this narrow interim power range we see a sharp increase in the RLF of Zone 1 as we move to lower radio powers, suggesting a boosted starforming population.

\subsubsection{Colour Analysis of Radio-Weak Sources}

Throughout we have referred to a cutoff power $\log \mathrm{P}_{1.4 c u t o f f} \approx 22.8$, that divides a SFdominated regime below, from an AGN-dominated regime above. However, sources below this level can still be AGNs (and vice versa); this just becomes less likely the further we move from the cutoff. Looking at the colours of sources is an additional indicator of SF behaviour, as this process emits thermal radiation in the blue part of the optical spectrum; 


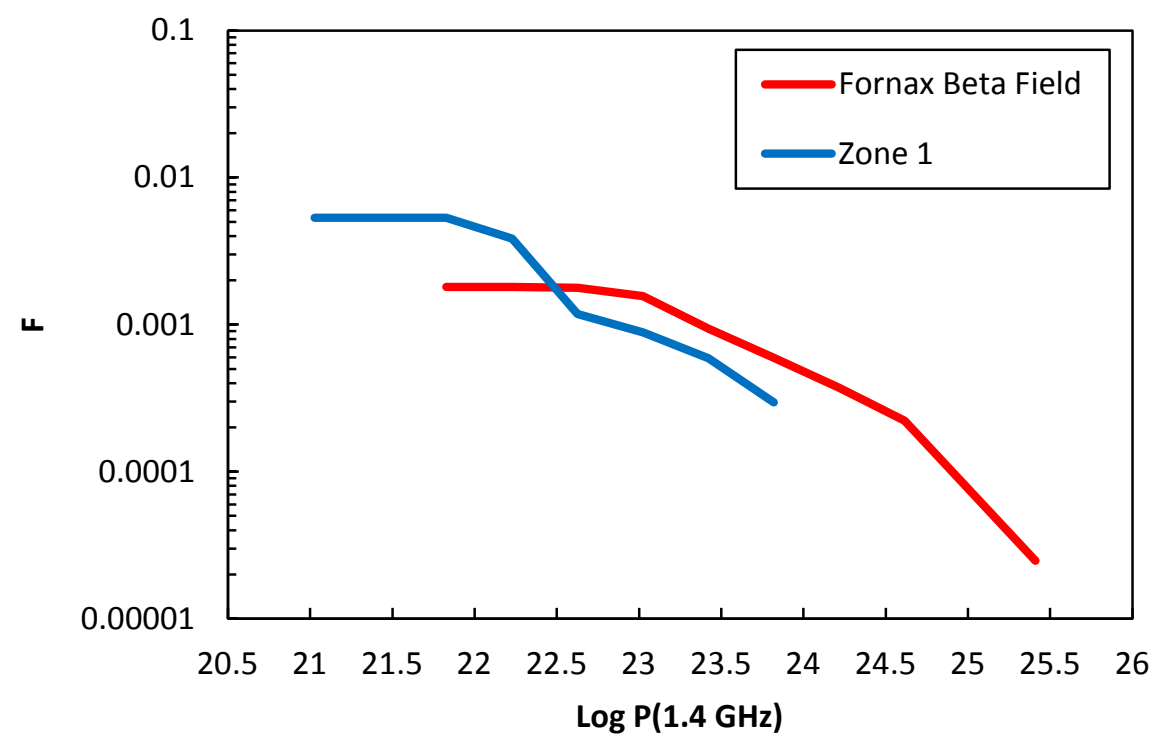

Figure 4.8: Both the Fornax Beta Field survey and the Zone 1 field observations were complete to much fainter magnitudes than the $\mathrm{b}_{J}=18.05$, as used in the comparison plot in Fig. 4.7. A more localised, but a more robust comparison can be achieved by using RLF lines from these two populations only, now at $\mathrm{b}_{J} \leq 22$ (the magnitude limit of the Fornax Field population). This allows for a statistically stronger parent population from each field; 73 Fornax Field sources, and 18 sources from Zone 1 constitute the functions.

\begin{tabular}{cccccc}
\hline$\Delta \log \mathrm{P}_{1.4}$ & $\Delta \mathrm{S}_{1.4}$ & Fractional BRLF & Integral BRLF \\
\hline $21.03-21.43$ & $0.13-0.33$ & $0 / 3384$ & 0 & $18 / 3384$ & 0.00532 \\
$21.43-21.83$ & $0.33-0.82$ & $5 / 3384$ & 0.00147 & $18 / 3384$ & 0.00532 \\
$21.83-22.23$ & $0.82-2.06$ & $9 / 3384$ & 0.00270 & $13 / 3384$ & 0.00384 \\
$22.23-22.62$ & $2.06-5.15$ & $1 / 3384$ & 0.00029 & $4 / 3384$ & 0.00118 \\
$22.62-23.02$ & $5.15-12.9$ & $1 / 3384$ & 0.00029 & $3 / 3384$ & 0.00088 \\
$23.02-23.42$ & $12.9-32.2$ & $1 / 3384$ & 0.00029 & $2 / 3384$ & 0.00059 \\
$23.42-23.82$ & $32.2-80.5$ & $1 / 3384$ & 0.00029 & $1 / 3384$ & 0.00029 \\
$23.82-24.22$ & $80.5-201$ & $0 / 3384$ & 0 & $0 / 3384$ & 0 \\
\hline
\end{tabular}

Table 4.2: Details of the bivariate radio luminosity function generated from the Zone 1 population with an optical magnitude cutoff of $\mathrm{b}_{J}=22$. 


\begin{tabular}{cccccc}
\hline$\Delta \log \mathrm{P}_{1.4}$ & $\Delta \mathrm{S}_{1.4}$ & Fractional BRLF & \multicolumn{3}{c}{ Integral BRLF } \\
\hline $21.43-21.83$ & $0.33-0.82$ & $0 / 40468$ & 0 & $73 / 40468$ & 0.00180 \\
$21.83-22.23$ & $0.82-2.06$ & $1 / 40468$ & 0.00002 & $73 / 40468$ & 0.00180 \\
$22.23-22.62$ & $2.06-5.15$ & $9 / 40468$ & 0.00022 & $72 / 40468$ & 0.00178 \\
$22.62-23.02$ & $5.15-12.9$ & $25 / 40468$ & 0.00062 & $63 / 40468$ & 0.00156 \\
$23.02-23.42$ & $12.9-32.2$ & $14 / 40468$ & 0.00035 & $38 / 40468$ & 0.00094 \\
$23.42-23.82$ & $32.2-80.5$ & $9 / 40468$ & 0.00022 & $24 / 40468$ & 0.00059 \\
$23.82-24.22$ & $80.5-201$ & $6 / 40468$ & 0.00015 & $15 / 40468$ & 0.00037 \\
$24.22-24.61$ & $201-503$ & $6 / 40468$ & 0.00015 & $9 / 40468$ & 0.00022 \\
$24.61-25.01$ & $503-1260$ & $2 / 40468$ & 0.00005 & $3 / 40468$ & 0.00007 \\
$25.01-25.41$ & $1260-3140$ & $1 / 40468$ & 0.00002 & $1 / 40468$ & 0.00002 \\
\hline
\end{tabular}

Table 4.3: Details of the bivariate radio luminosity function generated from the Fornax Beta Field population with an optical magnitude cutoff of $\mathrm{b}_{J}=22$.

elliptical galaxies are cooler and emit in the red. A first-order analysis was attempted with the SuperCOSMOS catalogue for Zone 1. The full optical catalogue was limited to those sources that had magnitudes $\mathrm{b}_{J} \leq 20$, leaving 1020 of the initial 21072 objects. This was done to minimise the presence of dim background sources that obscure the red cluster sequence (Gladders and Yee 2000). For these sources the blue excess (B-R) was plotted against the red magnitude R (see Fig. 4.9). In this population we see some evidence of the red sequence noted in Gladders and Yee, and represented in Fig. 4.9 by a region bounded by dotted blue lines. An optical source can be considered to exhibit a blue excess if it is found below this region on the graph. Onto the plot have been added blue and red magnitude data for six sources in the SuperCOSMOS catalogue (still with $b_{J} \leq 20$ ) for which there were radio counterparts identified in the Zone 1 mosaic map. All of these galaxies had radio powers that would place them in the SF-dominated regime. Of these sources, four are well below the red sequence in Fig. 4.9, with a fifth marginally below it. The final source is located marginally above the sequence. Because this result is drawn from only six sources it alone is not conclusive. However it suggests agreement with the trend seen in the RLF analysis for Zone 1 (Section 4.1.2) that this filament exhibits an excess of low powered radio sources, and that this is probably associated with a predominantly starforming population

Similar analysis was again unable to be performed for the population of Zone 2. Of the full SuperCOSMOS catalogue for that region, only three sources had radio counterparts, and none of these with magnitude $\leq 20$.

\subsubsection{Spectral Indices}

One benefit of CABB's $2 \mathrm{GHz}$ bandwidth is that splitting the band allows investigation of radio properties over frequency space with substantial spread between points. An example is spectral index analysis, in which we consider the integrated flux of a radio source at two or more frequencies. For extended radio sources we expect that the flux will decrease as we move to higher frequencies, as a result of electron aging in radio jets over galactic timescales. This allows us to define the spectral index $\alpha$ for a radio source, as per Eqn 2.1. This definition implies that $\alpha$ is a constant associated with each source. In reality the spectral index is 


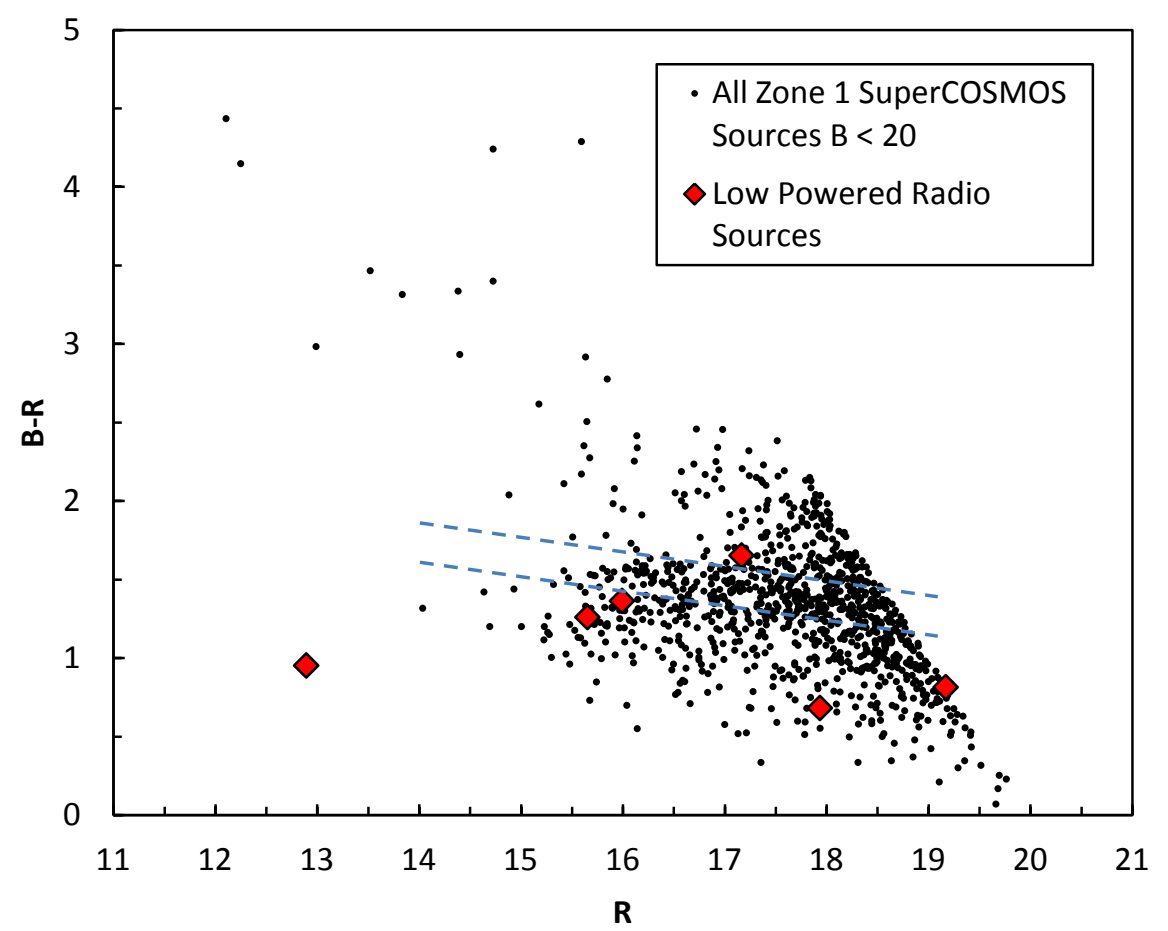

Figure 4.9: Blue and red magnitude measures plotted for optical sources drawn from the SuperCOSMOS catalogue for Zone 1 . The sources were limited to only those with $\mathrm{b}_{J} \leq 20$. From this reduced optical catalogue, those sources with radio catalogues were identified (red diamonds). Only one radio source is found on the red side of the red sequence (Gladders and Yee 2000), with the remainder showing some degree blue excess. This suggests that in the low power regime, the source overdensity in Zone 1 is primarily due to SF galaxies. 
itself a continuous function of frequency. Because we typically measure the flux of a source at several discrete frequencies we often treat $\alpha$ as a piecewise function that has a constant value between frequency pairs. As most spectral indices remain fairly constant across an appropriate frequency range, this is considered an acceptable compromise. Note also that in interferometric observations the sensitivity to different angular power-scales does vary with frequency. In practice both effects would be present in wide-band interferometric imaging.

So again we are faced with a trade-off. On one hand, we would like to split our observing band into the maximum number of subbands possible to achieve the highest-order approximation of the continuous $\alpha_{\nu 1}^{\nu 2}$ function. However, every additional subband split will result in a loss of sensitivity - meaning fewer sources will be detected above the noise - as well as increasing the work required in the data reduction phase.

It was decided to split the band into four again for spectral index analysis. The benefit of this was that the four subbands had already been reduced, so required very little preparation before spectral indices could be generated. All maps for both fields were reduced in size using cgcurs to agree with the region selection of the full-frequency-band images (Section 3.1.3). The Duchamp source finding algorithm was then applied to all images, providing separate source catalogues for each frequency band. The catalogued sources were matched, using the full-frequency catalogues as a reference, with the TOPCAT source matching package. The assumption was that, due to its deeper sensitivity, any sources detected in any subband would also have been detected in the master map, so the master catalogue ID was used as the unique identifier for sources. Sources from separate bands with a spatial separation of less than 1.5 arcsec were considered to be the same object. TOPCAT allows matches to be generated from up to five worksheets, which is the number we had to deal with. That is one issue that would have to be overcome if we were interested in generating more than three spectral index values for each source (n spectral indices requiring $(n+1)$ flux measurements plus the reference catalogue position). Because sources had to show up in the catalogues for different frequencies, there was less likelihood of false detection. After the catalogues were matched with TOPCAT the detections were checked on the relavant master mosaic. For each zone one source was identified as spurious, and removed from the catalogue.

The spectral indices of all sources for which there were flux measurements at at least two frequencies, are laid out on $\log \mathrm{S}-\log \nu$ plots in Figs. 4.10 and 4.11 respectively. For Zone 1 there are 56 sources plotted, and a further 33 for Zone 2. These are split into several frames for clarity. Additionally the sources are listed in Tables 4.4 and 4.5 (note because the spectral indices here were drawn from sources with flux measures from adjacent bands, the tables contain only 50 and 31 sources, respectively). Interestingly, comparing the spectral indices of the two Zones shows an apparent difference in the overall trend. Most Zone 1 sources seem to have spectra that are relatively flat or with a shallow positive gradient ( $\alpha \leq 0$ under the sign convention used here). In contrast with this, the majority of Zone 2 sources display what could be considered more traditional spectral indices, where $\alpha \geq 0$. Some sources here exhibit some steepening (more common) or shallowing (less common) in their spectra at higher frequencies, though for the most part this is small enough to cause only minor deviation in the plot line.

It has been proposed that radio sources in clusters may exhibit steeper spectral indices than comparable field sources because of confinement of radiating electrons by the intracluster 
medium (Roland et al. 1985). The spectral indices of Zone 1, being flatter, are more consistent with this theory than those of Zone 2 - most of which are $\geq 1$.

It was considered whether the difference in spectral indices between Zones 1 and 2 were due to the kinds of sources that were being detected. To investigate the source morphologies, the field mosaics were again inspected. Not surprisingly, the vast majority of galaxies were point source detections (see Tables 4.4 and 4.5). For Zone 2 only one source (Source 28) showed any sign of extended morphology. In Zone 1 there was a little more variety. Five sources were extended $(9,21,31,39$ and 47), with a further four (20,25, 34 and 40) being pairs of (point source) galaxies that Duchamp had picked up as single sources. However, we saw in Figs. 4.10 and 4.11 the disparity between the Zones was evident in most sources, and the bulk of these apparently share the same morphological type.

Variation in the spectral indices of sources between different frequency pairs can be described visually by using a colour-colour plot, where the higher-band index is plotted against the lower. Normally to do this we would have flux measurements at three frequencies, giving a pair of spectral indices for which the choice of plot axes is trivial. As mentioned above we have four frequencies, hence three spectral index values, and so we have three options of pairs of indices we could plot. So as not to throw any data away here all three plots were generated for sources from each field (see Figs. 4.12 and 4.13). The spectral index pairs in the figures is $\alpha_{1844}^{2356}$ vs $\alpha_{1332}^{1844}$ in the upper pane, $\alpha_{2356}^{2868}$ vs $\alpha_{1844}^{2356}$ in the centre, and $\alpha_{2356}^{2868}$ vs $\alpha_{1332}^{1844}$ in the lower pane. For reference on these plots a $\mathrm{x}=\mathrm{y}$ line has been added. Sources on this line have a spectral index that doesn't change as we move from lower to higher frequency. Because the first two plots for each field show spectral indices from adjacent frequency pairs, we would expect the difference from one to the other to be less dramatic than in the lower plot, in which the indices plotted are from either end of the full $2 \mathrm{GHz}$ band.

\section{Investigation of Calibration Error in Zone 1 Data}

Again we see far more reasonable behaviour in the colour-colour plots for Zone 2 than for Zone 1. Calibration error in the initial data reduction for Zone 1 was considered as a final avenue for the apparent difference in the source population. If the calibration process was confirmed to have been entirely successful, it must be concluded that the disparity in spectral index properties between the two zones is a real effect.

To test the calibration, the flux of primary and secondary calibrators were drawn from the raw data of Zones 1 and 2 at the four subbands. From these flux measures a spectral index was generated for the sources, this being the average spectral index across the $2 \mathrm{GHz}$ band. It was found that while the primary calibrator PKS B1934-638 had the same spectral index for both zones $(0.506 \pm 0.001)$, the secondary calibrators showed markedly different properties. The secondary calibrator for Zone 2, 0438-436 had a measured spectral index of $\alpha_{a v}=0.58 \pm 0.02$. This value is slightly shallower than the index of $\sim 0.7$ expected for a typical radio source, but it is not unreasonable. Conversely, from the raw data 0302-623, the Zone 1 secondary appeared to have a spectral index of $\alpha_{a v}=0.040_{-0.013}^{+0.029}$. This value is unusually shallow for a normal radio source. It is also similar to the very shallow indices observed for the majority of Zone 1 sources (see Table 4.4 and Fig. 4.10).

Of course, the secondary calibrators for Zones 1 and 2 were different sources, so probably 

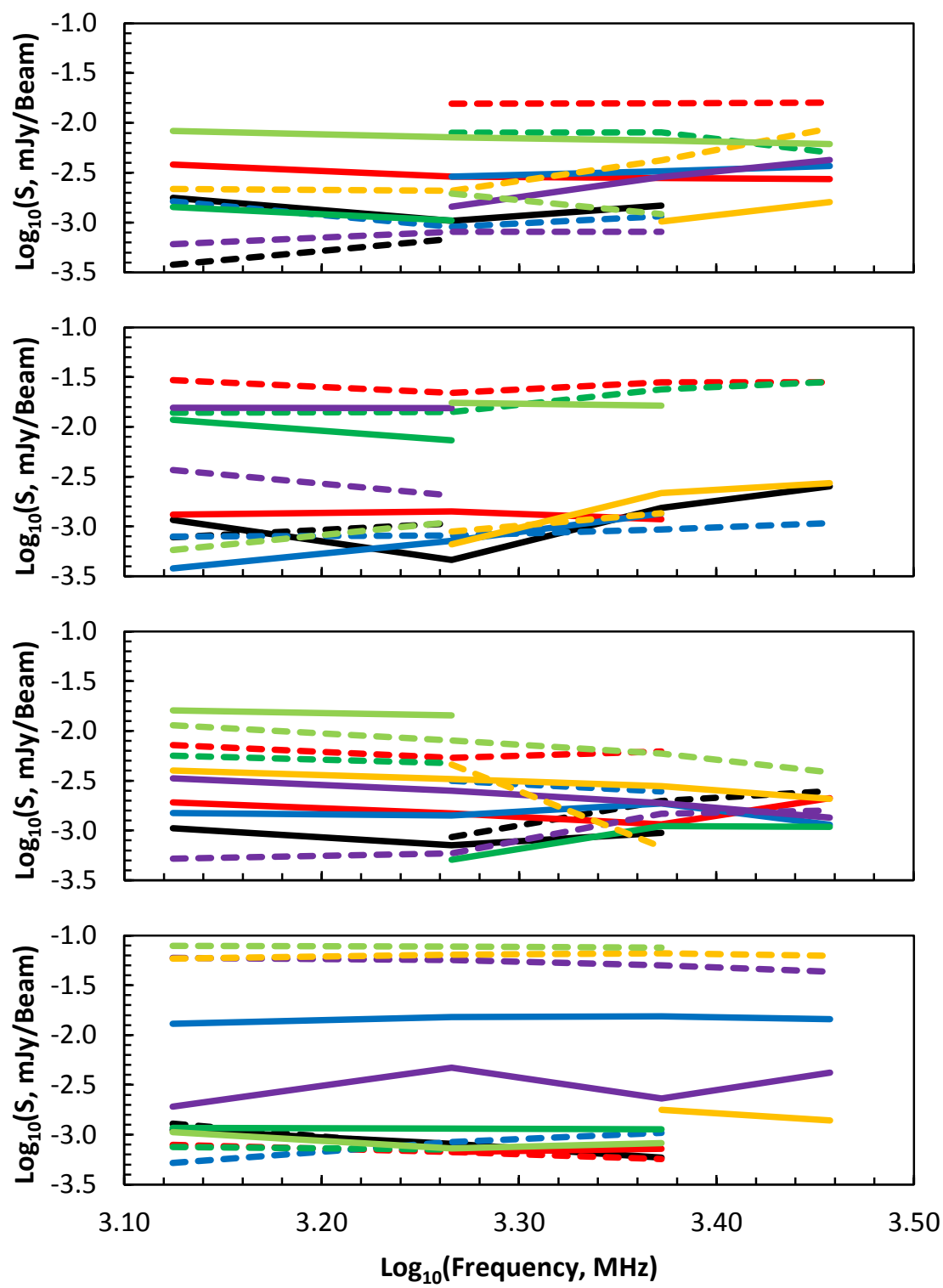

Figure 4.10: Spectral index plots for all Zone 1 sources with at least two measured flux values. The fluxes at each band are represented on a log-log plot, from which the gradient defines the (negative) spectral index, according to Eqn. 2.1. Spectra for 56 sources have been divided into 4 plots for clarity. 

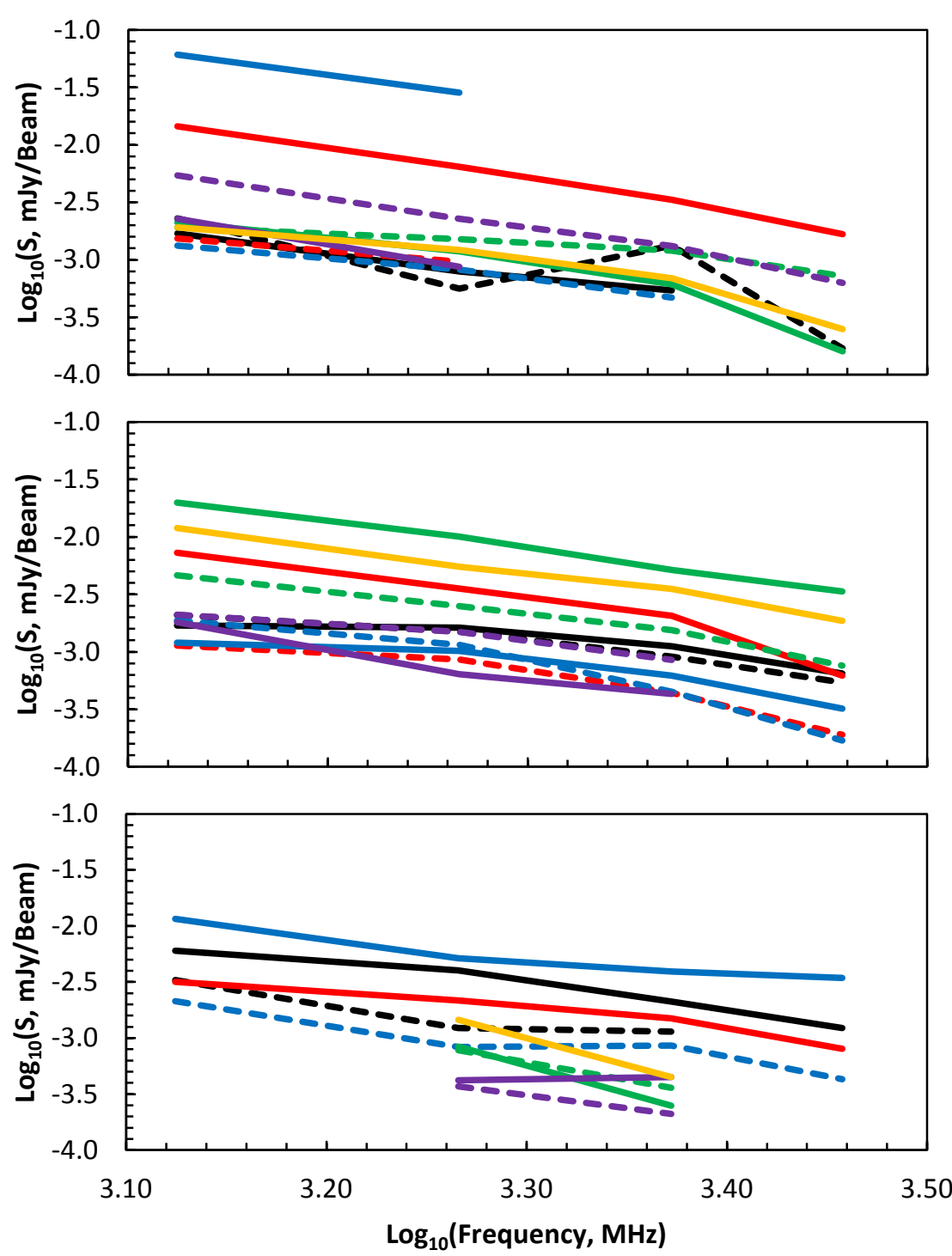

Figure 4.11: Spectral index plots for all Zone 2 sources with at least two measured flux values. Spectra for 33 sources have been divided into 3 plots for clarity. 


\begin{tabular}{|c|c|c|c|c|c|c|c|c|c|c|}
\hline $\begin{array}{r}\text { Source } \\
\text { No. }\end{array}$ & $\begin{array}{c}\text { Duchamp } \\
\text { Catalogue ID }\end{array}$ & $\begin{array}{c}\text { Position } \\
\text { RA }\end{array}$ & $\begin{array}{c}(\mathrm{J} 2000) \\
\text { DEC }\end{array}$ & $\begin{array}{l}\mathrm{S}_{1332} \\
(\mathrm{mJy})\end{array}$ & $\begin{array}{l}\mathrm{S}_{1844} \\
(\mathrm{mJy})\end{array}$ & $\begin{array}{l}\mathrm{S}_{2356} \\
(\mathrm{mJy})\end{array}$ & $\begin{array}{l}\mathrm{S}_{2868} \\
(\mathrm{mJy})\end{array}$ & $\alpha_{1332}^{1844}$ & $\alpha_{1844}^{2356}$ & $\alpha_{2356}^{2868}$ \\
\hline 1 & M2013 J053.416-53.720 & 53.4162 & -53.7197 & 0.069 & 0.072 & 0.084 & & -0.13 & -0.63 & \\
\hline 2 & M2013 J053.422-53.692 & 53.4218 & -53.6922 & 0.036 & 0.061 & & & -1.62 & & \\
\hline 3 & M2013 J053.466-53.569 & 53.4663 & -53.5692 & 0.080 & 0.106 & & 0.140 & -0.87 & & \\
\hline 4 & M2013 J053.477-53.575 & 53.4767 & -53.5751 & 0.062 & 0.078 & 0.080 & & -0.71 & -0.10 & \\
\hline 5 & M2013 J053.477-53.713 & 53.4770 & -53.7132 & 0.061 & 0.084 & & & -0.98 & & \\
\hline 6 & M2013 J053.540-53.656 & 53.5404 & -53.6560 & & 0.137 & 0.161 & 0.180 & & -0.66 & -0.57 \\
\hline 7 & M2013 J053.550-53.662 & 53.5503 & -53.6619 & & 0.089 & 0.116 & 0.190 & & -1.08 & \\
\hline 8 & M2013 J053.561-53.081 & 53.5613 & -53.0811 & 0.046 & 0.069 & 0.073 & & -1.25 & -0.23 & \\
\hline 9 & M2013 J053.600-53.676 & 53.6000 & -53.6755 & & & 0.087 & 0.150 & & & -2.77 \\
\hline 10 & M2013 J053.601-52.760 & 53.6010 & -52.7596 & 0.069 & 0.097 & 0.139 & 0.280 & -1.05 & -1.47 & -3.56 \\
\hline 11 & M2013 J053.647-53.677 & 53.6472 & -53.6766 & & 0.089 & 0.080 & & & 0.44 & \\
\hline 12 & M2013 J053.655-52.820 & 53.6554 & -52.8205 & 0.116 & 0.162 & & 0.220 & -1.03 & & \\
\hline 13 & M2013 J053.657-53.682 & 53.6568 & -53.6817 & 0.059 & 0.061 & 0.094 & 0.140 & -0.10 & -1.76 & -2.03 \\
\hline 14 & M2013 J053.664-52.984 & 53.6640 & -52.9836 & 0.046 & 0.081 & & & -1.74 & & \\
\hline 15 & M2013 J0 & 53.7213 & -52.9825 & 0.051 & 0.089 & 0.080 & & -1.71 & 0.44 & \\
\hline 16 & M2013 J( & 53.7429 & -53.1195 & 0.118 & 0.182 & 0.233 & 0.330 & -1.33 & -1.01 & -1.77 \\
\hline 17 & M2013 J053.784-52.879 & 53.7837 & -52.8790 & 0.044 & 0.072 & & 0.110 & -1.51 & & \\
\hline 18 & M2013 J053.794-53.256 & 53.7938 & -53.2557 & 0.117 & 0.172 & & & -1.18 & & \\
\hline 19 & M2013 J053.8 & 53.8532 & -53.0752 & 0.105 & 0.136 & 0.218 & 0.300 & -0.80 & -1.93 & -1.62 \\
\hline 20 & M20 & 53.8740 & -53.5642 & 0.118 & 0.194 & & & -1.53 & & \\
\hline 21 & M2013 J053.8 & 53.8805 & -53.4557 & 0.103 & 0.119 & & & -0.44 & & \\
\hline 22 & M2013 J053.948-53.855 & 53.9479 & -53.8551 & & 0.072 & 0.106 & 0.150 & & -1.58 & -1.77 \\
\hline 23 & M2013 J053.953-53.301 & 53.9530 & -53.3014 & & 0.078 & 0.094 & & & -0.76 & \\
\hline 24 & M2013 J0 & 53.9901 & -53.8477 & 0.038 & 0.069 & & & -1.83 & & \\
\hline 25 & M2013 JC & 54.0080 & -53.8466 & & 0.268 & 0.284 & & & -0.24 & \\
\hline 26 & M2013 J054.2 & 54.2422 & -53.3775 & & 0.075 & 0.097 & 0.130 & & -1.05 & -1.49 \\
\hline 27 & M2013 J054.242-53.483 & 54.2417 & -53.4827 & 0.058 & 0.072 & 0.080 & & -0.66 & -0.43 & \\
\hline 28 & M2013 J054.250-52.853 & 54.2502 & -52.8531 & 0.067 & & 0.077 & 0.150 & & & -3.39 \\
\hline 29 & M20 & 54.2776 & -53.7552 & 0.108 & 0.152 & 0.157 & & -1.05 & -0.13 & \\
\hline 30 & M2013 JC & 54.2966 & -53.5847 & 0.061 & 0.092 & 0.103 & 0.110 & -1.26 & -0.46 & -0.33 \\
\hline 31 & M2013 J054.2 & 54.2979 & -52.8903 & & 0.147 & 0.146 & & & 0.03 & \\
\hline 32 & M2013 J054.3 & 54.3845 & -53.6135 & & 0.065 & 0.084 & 0.110 & & -1.05 & -1.37 \\
\hline 33 & M2013 JC & 54.4000 & -53.7273 & 0.139 & 0.207 & & & -1.22 & & \\
\hline 34 & M2013 J054.4 & 54.4299 & -53.6665 & 0.095 & 0.142 & & & -1.24 & & \\
\hline 35 & M2013 J054.438-53.536 & 54.4382 & -53.5362 & 0.078 & 0.104 & 0.094 & 0.120 & -0.88 & 0.41 & -1.24 \\
\hline 36 & M2013 J054.518-53.234 & 54.5176 & -53.2339 & 0.046 & 0.065 & 0.087 & & -1.06 & -1.19 & -1 \\
\hline 37 & M2013 JC & 54.5306 & -53.5330 & 0.087 & 0.115 & 0.108 & 0.1 & -0.86 & 0.26 & -0 \\
\hline 38 & M2013 J0 & 54.5355 & -53.5944 & 0.124 & 0.175 & 0.157 & 0.190 & -1.06 & 0.44 & -0.97 \\
\hline 39 & M2013 J054.5! & 54.5926 & -53.6388 & 0.116 & 0.182 & & & -1.38 & & \\
\hline 40 & M2013 J054.615-53.773 & 54.6153 & -53.7730 & 0.055 & 0.061 & & & -0.32 & & \\
\hline 41 & M2013 J054.617-53.263 & 54.6172 & -53.2632 & & 0.072 & 0.073 & & & -0.06 & \\
\hline 42 & M2013 J054.713-53.409 & 54.7125 & -53.4093 & 0.100 & 0.160 & 0.152 & 0.190 & -1.45 & 0.21 & -1.13 \\
\hline 43 & M2013 J054.754-53.694 & 54.7539 & -53.6943 & 0.044 & 0.075 & 0.077 & & -1.64 & -0.11 & \\
\hline 44 & M2013 J054.810-53.596 & 54.8102 & -53.5957 & 0.054 & 0.072 & & & -0.88 & & \\
\hline 45 & M2013 J054.818-53.088 & 54.8181 & -53.0884 & 0.061 & 0.150 & 0.103 & 0.180 & -2.77 & 1.53 & -2.84 \\
\hline 46 & M2013 J054.8 & 54.8863 & -53.2996 & 0.145 & 0.234 & 0.210 & 0.300 & -1.47 & 0.44 & -1.81 \\
\hline 47 & M2013 J054.904-53.226 & 54.9043 & -53.2259 & & & 0.097 & 0.110 & & & -0.64 \\
\hline 48 & M2013 J054.918-53.243 & 54.9180 & -53.2434 & 0.128 & 0.223 & 0.220 & 0.240 & -1.71 & 0.06 & -0.44 \\
\hline 49 & M2013 J054.931-53.109 & 54.9311 & -53.1095 & 0.141 & 0.288 & 0.266 & & -2.20 & 0.32 & \\
\hline 50 & M2013 J054.935-53.426 & 54.9353 & -53.4263 & 0.058 & 0.072 & 0.077 & & -0.66 & -0.27 & \\
\hline
\end{tabular}

Table 4.4: Relative measured fluxes at four frequency subbands for 50 Zone 1 (above) and 31 Zone 2 (overleaf) sources with a minimum of one spectral index value (requiring a pair of neighbouring flux measures). Flux values are taken from Duchamp-generated catalogues for each band, and then combined with the master $(2100 \mathrm{MHz})$ catalogue via TOPCAT. Catalogue IDs are from the master catalogue. Spectral indices from these tables form the basis of the colour-colour plots in Figs. 4.12 and 4.13. 


\begin{tabular}{|c|c|c|c|c|c|c|c|c|c|c|}
\hline $\begin{array}{r}\text { Source } \\
\text { No. }\end{array}$ & $\begin{array}{c}\text { Duchamp } \\
\text { Catalogue ID } \\
\end{array}$ & $\begin{array}{l}\text { Position } \\
\text { RA } \\
\end{array}$ & $\begin{array}{c}(\mathrm{J} 2000) \\
\text { DEC }\end{array}$ & $\begin{array}{l}\mathrm{S}_{1332} \\
(\mathrm{mJy})\end{array}$ & $\begin{array}{l}\mathrm{S}_{1844} \\
(\mathrm{mJy})\end{array}$ & $\begin{array}{l}\mathrm{S}_{2356} \\
(\mathrm{mJy})\end{array}$ & $\begin{array}{l}\mathrm{S}_{2868} \\
(\mathrm{mJy})\end{array}$ & $\alpha_{1332}^{1844}$ & $\alpha_{1844}^{2356}$ & $\alpha_{2356}^{2868}$ \\
\hline 1 & M2013 J053.420-38.364 & 53.4198 & -38.3637 & 0.132 & 0.064 & 0.039 & & 2.23 & 2.02 & \\
\hline 2 & M2013 J053.434-38.404 & 53.4340 & -38.4036 & 0.163 & 0.052 & 0.063 & 0.023 & 3.51 & -0.78 & 5.12 \\
\hline 3 & M2013 J053.570-38.395 & 53.5695 & -38.3950 & 0.243 & 0.099 & 0.059 & 0.049 & 2.76 & 2.11 & 0.94 \\
\hline 4 & M2013 J053.603-38.565 & 53.6035 & -38.5645 & 0.132 & 0.074 & & & 1.78 & & \\
\hline 5 & M2013 J053.630-38.205 & 53.6297 & -38.2054 & & 0.064 & 0.029 & & & 3.23 & \\
\hline 6 & M2013 J053.662-38.577 & 53.6619 & -38.5769 & & 0.064 & 0.033 & & & 2.70 & \\
\hline 7 & M2013 J053.675-38.064 & 53.6752 & -38.0640 & 0.233 & 0.115 & & & 2.17 & & \\
\hline 8 & M2013 J053.712-38.745 & 53.7120 & -38.7454 & & 0.047 & 0.035 & & & 1.20 & \\
\hline 9 & M2013 J053.752-38.681 & 53.7516 & -38.6806 & 0.122 & 0.068 & 0.039 & & 1.80 & 2.27 & \\
\hline 10 & M2013 J053.774-38.544 & 53.7741 & -38.5435 & 0.140 & 0.072 & 0.040 & 0.023 & 2.04 & 2.40 & 2.81 \\
\hline 11 & M2013 J053. & 53.8397 & -39.1615 & 0.132 & 0.078 & 0.050 & 0.042 & 1.62 & 1.81 & 0.89 \\
\hline 12 & M2013 J053.862-38.508 & 53.8622 & -38.5084 & 0.148 & 0.070 & & & 2.30 & & \\
\hline 13 & M2013 J053.882-38.156 & 53.8815 & -38.1564 & 0.189 & 0.087 & 0.049 & 0.038 & 2.39 & 2.34 & 1.29 \\
\hline 14 & M2013 J053.906-38.279 & 53.9056 & -38.2792 & & 0.047 & 0.027 & & & 2.26 & \\
\hline 15 & M2013 J053. & 53.9238 & -38.1433 & 0.136 & 0.074 & 0.040 & 0.026 & 1.87 & 2.51 & 2.19 \\
\hline 16 & M2013 J053.991-38.753 & 53.9911 & -38.7531 & 0.127 & 0.079 & 0.049 & 0.039 & 1.46 & 1.95 & 1.16 \\
\hline 17 & M2013 J053.998-38.289 & 53.9976 & -38.2885 & 0.140 & 0.076 & 0.044 & 0.036 & 1.88 & 2.23 & 1.02 \\
\hline 18 & M2013 J054.008-38.603 & 54.0076 & -38.6034 & 0.204 & 0.101 & 0.063 & 0.040 & 2.16 & 1.93 & 2.31 \\
\hline 19 & M2013 J054.064-38.604 & 54.0644 & -38.6042 & 0.113 & 0.066 & 0.035 & 0.024 & 1.65 & 2.59 & 1.92 \\
\hline 20 & M2013 J054. & 54.0957 & -39.1596 & 0.107 & 0.068 & 0.042 & 0.031 & 1.39 & 1.97 & 1.54 \\
\hline 21 & M2013 J054.106-38.887 & 54.1061 & -38.8870 & 0.136 & 0.072 & 0.036 & 0.023 & 1.96 & 2.83 & 2.28 \\
\hline 22 & M2013 J054.164-38.182 & 54.1637 & -38.1824 & 0.250 & 0.107 & 0.062 & 0.057 & 2.61 & 2.23 & 0.43 \\
\hline 23 & M2013 J054.169-38.302 & 54.1693 & -38.3019 & 0.176 & 0.087 & 0.051 & 0.041 & 2.17 & 2.18 & 1.11 \\
\hline 24 & M2013 J054.205-37.889 & 54.2053 & -37.8888 & 0.140 & 0.057 & 0.036 & & 2.76 & 1.88 & \\
\hline 25 & M2013 J054.206-38.855 & 54.2063 & -38.8554 & 0.136 & 0.081 & 0.046 & & 1.59 & 2.31 & \\
\hline 26 & M2013 J054.231-38.170 & 54.2312 & -38.1700 & 0.209 & 0.094 & 0.061 & 0.049 & 2.46 & 1.76 & 1.11 \\
\hline 27 & M2013 J054.234-39.036 & 54.2343 & -39.0365 & 0.186 & 0.103 & 0.061 & 0.051 & 1.82 & 2.14 & 0.91 \\
\hline 28 & M2013 J054.254-37.973 & 54.2541 & -37.9734 & 0.180 & 0.086 & 0.059 & & 2.27 & 1.54 & \\
\hline 29 & M2013 J054.294-38.125 & 54.2942 & -38.1251 & 0.148 & 0.083 & 0.053 & 0.041 & 1.78 & 1.83 & 1.31 \\
\hline 30 & M2013 J054.510-37.916 & 54.5103 & -37.9161 & 0.186 & 0.095 & 0.067 & 0.071 & 2.07 & 1.43 & -0.29 \\
\hline 31 & M2013 J054.540-38.036 & 54.5396 & -38.0358 & 0.136 & 0.060 & 0.043 & 0.032 & 2.52 & 1.36 & 1.50 \\
\hline
\end{tabular}

Table 4.5: Spectral index data for 31 Zone 2 sources. Further explanation previous page. 

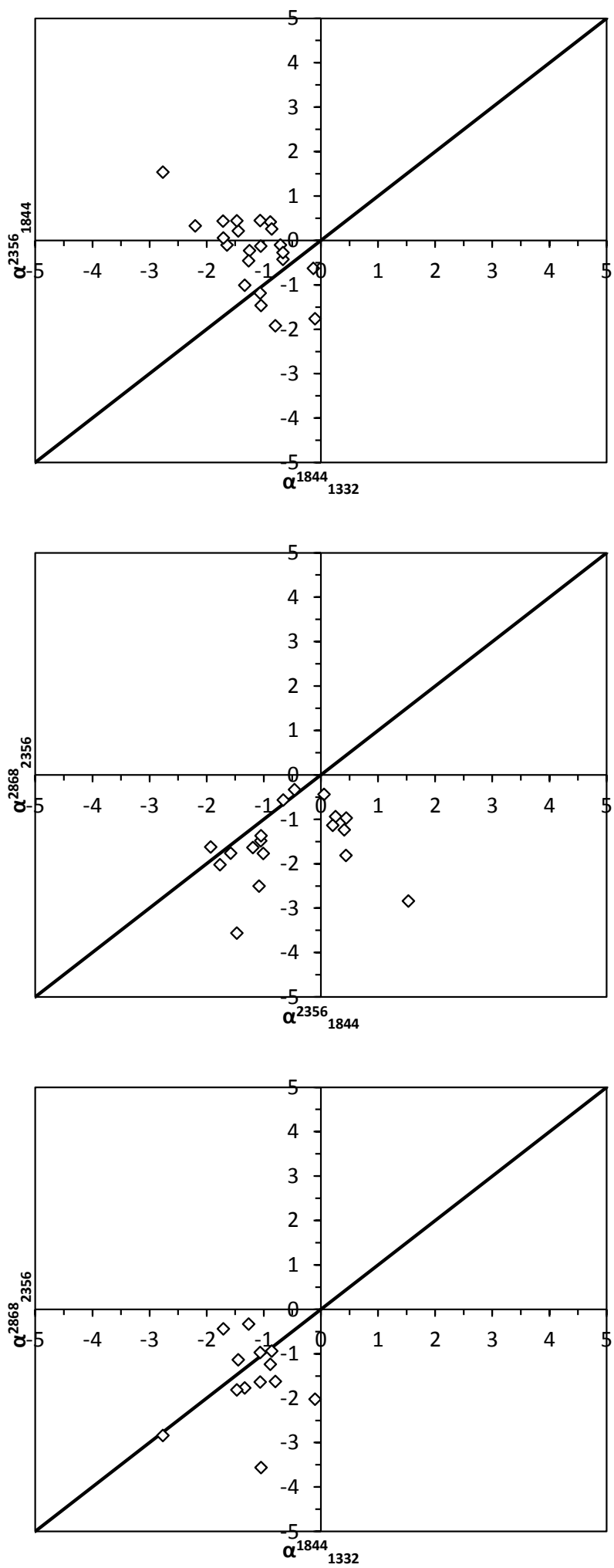

Figure 4.12: Colour-colour plots for spectral index measures of Zone 1 sources. These plots compare the spectral index of a higher frequency band with that of a lower band. A maximum of three index measures for each source give us three options of pair comparisions. Spectral indices that do not change between bands will appear as points on the $\mathrm{x}=\mathrm{y}$ line added for reference. Here the points imply significant variation of spectral indices between frequency bands. 

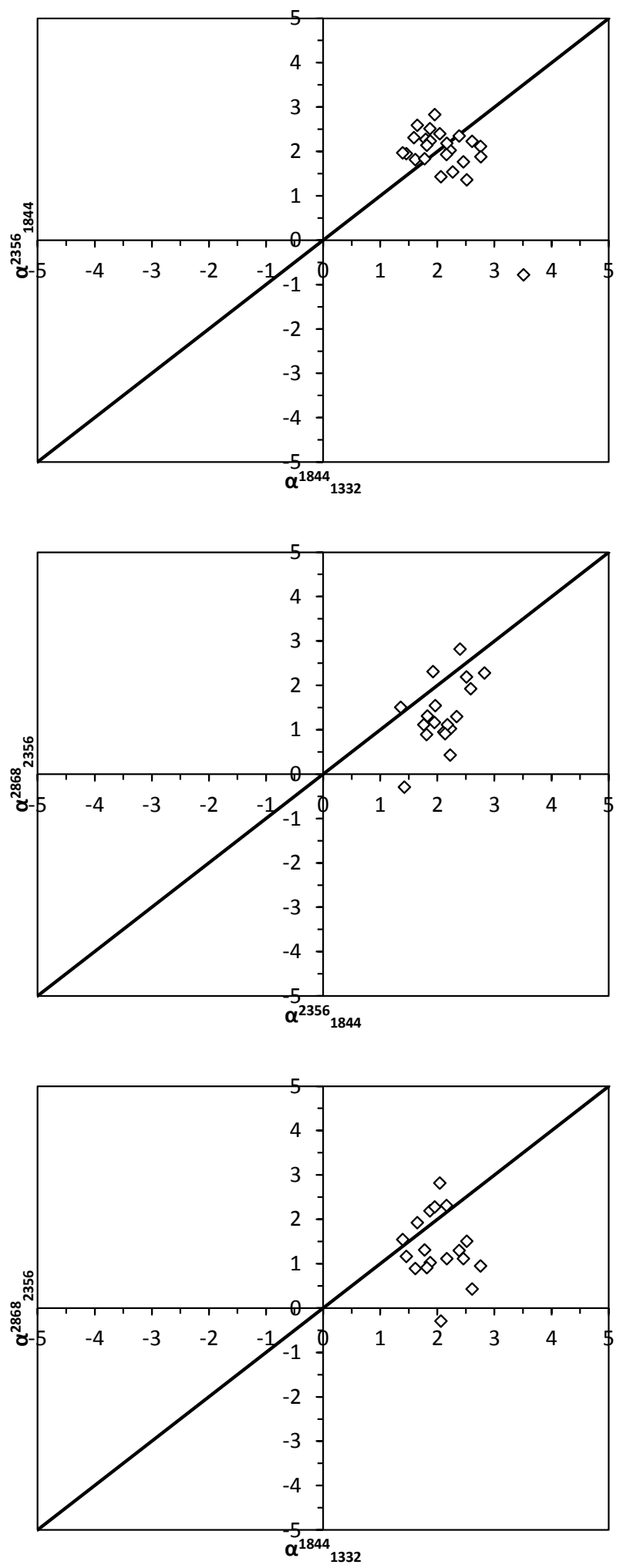

Figure 4.13: Colour-colour plots for spectral index measures of Zone 2 sources. Explanation on previous page. Here we see much tighter grouping about the $\mathrm{x}=\mathrm{y}$ line, implying spectral indices that are rather more constant across the full $2 \mathrm{GHz}$ band of our observations. 
ought not have identical spectral indices across the $2 \mathrm{GHz}$ band of our observations. As a first-order test of the reliability of our spectral index measures, the measured fluxes for both calibrators were drawn from the ATCA calibrator database ${ }^{2}$ at five of the six CABB observing bands (data for $3 \mathrm{~mm}$ observations were not used, these values significantly steepening the derived index measures). These ATCA bands, covering wavelengths $16 \mathrm{~cm}-7 \mathrm{~mm}$, are obviously spread over a far greater frequency range than our data, but offer some idea of a reasonable spectral index for our calibrators. From these data, 0438-436 was found to have a (database) spectral index of $\alpha_{d b}=0.7_{-0.3}^{+0.5}$, consistent with our observed value for Zone 2 . In contrast 0302-623 gave $\alpha_{d b}=0.4_{-0.2}^{+0.3}$. This figure is significantly different from our observed spectral index drawn from the Zone 1 data. It is also far more consistent with both 0438-436 and typical radio sources in general.

From this test it appears highly likely that the Zone 1 data has been affected by a significant error in the calibration process. Ideally it would be desirous to return to the calibration stage, isolate the problem, and then continue forward through the reduction process again. This would result in a more robust 4-band catalogue from which correct spectral indices could be calculated for radio sources in the region. Unfortunately there simply was not time for such a re-reduction within the scope of this project. A useful initial step however, is to artificially boost our subband fluxes by using the spectral index value drawn from the ATCA calibrator database. This would allow us to see whether recalibration would be likely to result in spectral indices for Zone 1 that are consistent with a typical radio population.

To boost the subband fluxes for the sources of Zone 1, we began with the measured value at $2868 \mathrm{MHz}$. This figure was kept as-is and to the remaining values was applied a spectral index of 0.4, this being the average index from the calibrator database. Any sources without a $2868 \mathrm{MHz}$ flux were removed from the catalogue for the purposes of this trial. The resulting corrected flux spectra for the remaining 24 sources were then plotted in Fig. 4.14.

As can be seen in Figure 4.14 boosting the flux values via a spectral index of 0.4 had very little observable effect on the overall trends of source spectra. All 24 sources still show spectra that are inverted for most of the band. In no case do we see a completely non-inverted spectrum. Referring to Fig. 4.10 it must be noted that those spectra with a $2868 \mathrm{MHz}$ flux measure seem to show a higher likelihood of being inverted, than those without. Additionally, the vertical scale here is smaller than before, exaggerating the steepness of spectra. However, the simple evidence here is that the anomalous spectra cannot be adequately described by boosting fluxes on this scale. The test was repeated for a spectral index of 0.7 , the upper limit implied from the flux data on the calibrator database. Even here the majority of spectra for Zone 1 sources remained inverted. In fact, it was found that the minimum spectral index $\alpha_{d b}$ required to boost the average derived index into a positive (non-inverted) regime was 1.14, significantly higher than expected for typical radio sources.

The colour-colour plots of Fig. 4.12 were repeated for the flux-boosted population. These can be seen in Fig. 4.15. What is clear from the figure is that, other than fewer points because of the reduced catalogue size, there is no significant difference from before in the observed trend. The boosting of flux has slightly shifted all points upwards and to the right. This is not surprising, as the boost served to make the spectral indices more positive everywhere. The correction has not affected the loose grouping of points on the plots. We certainly do

\footnotetext{
${ }^{2}$ http://www.narrabri.atnf.csiro.au/calibrators/
} 


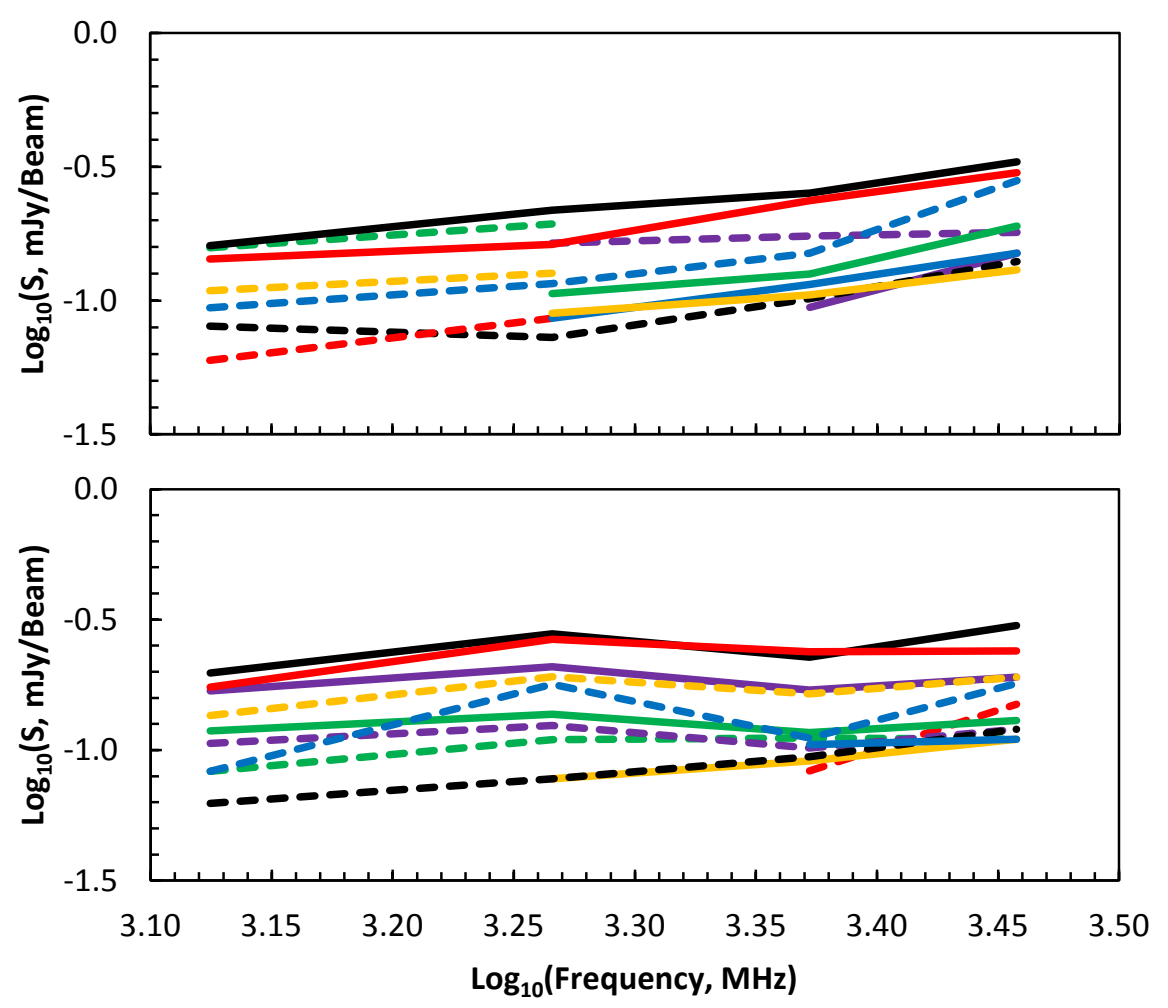

Figure 4.14: Spectral index plots to test the applied spectral index correction described in the text. Unfortunately the boost afforded by the additional index of 0.4 was not sufficient to reverse the inverted spectra evident in these sources. Here 24 spectra have been divided into two plots for clarity. 
not see the tightly bound groupings evident in the Zone 2 population (see Fig. 4.13). This effect also suggests that the issue is more serious than a simple spectral index substitution will remedy.

\subsection{Polarisation Properties of Zones 1 and 2}

\subsubsection{Generation of Polarisation Images}

In addition to the reduction of observational data into radio intensity maps for Zones 1 and 2 , the data was also reduced to generate maps of the polarisation of both fields. Here the reduction process was exactly the same as outlined in Section 3.1.3. The calibrated UV datasets for each field pointing at each frequency subband were run through the imaging script that had been used for radio intensity imaging, with an additional looping layer to create Stokes q, $u$ and $v$ images in one pass. The only other variation to the script was that the number of iterations of mfclean was reduced from 100000 to only 1000 because of the much lower noise level inherent in $\mathrm{q} / \mathrm{u} / \mathrm{v}$ images. This meant that the scripts ran much faster than the corresponding Stokes i reductions, even though the image output had been trebled.

The Stokes $\mathrm{q}$ and $\mathrm{u}$ images for each field at each frequency band were stitched together into a pair of mosaics using linmos, as had been done for the intensity maps. Finally, the q- and u-mosaics were combined together using impol, resulting in four polarisation maps for each filament zone; one for each frequency subband. Note that the q- and u- images for each pointing were mosaiced before the generation of the polarisation map. While we could alternately make p-images for each field, and then stitch those with linmos, the result would be incorrect. The root of this lies in the name of the routine; linmos combines linear properties. Intensity is a linear quantity, polarisation is not. This is also why we cannot simply combine the p-maps of all four subbands into a single master polarisation image for each zone easily. It can be done, but not with linmos. The final polarisation mosaics at 1844 $\mathrm{MHz}$ are presented in Figs. 4.16 and 4.17. The maps for the other frequency bands were not noticeably different, so have been omitted in the interests of brevity.

In the polarised emission mosaic map of Zone 1 (Fig. 4.16) we see polarised emission from a number of sources, but none of these are extended beyond $\sim 30$ ". In contrast, the Zone 2 map (Fig. 4.17) shows evidence of extended emission at either end (NE, SW) of the filament in this region. This polarised emission is coincident with the locations of clusters A3145 and A3135. It also appears to be diffuse emission, not associated with any discrete sources. What this suggests is that we have detected clusters by observing the polarised signatures left by their magnetic fields. Although this was not central to the aims of this study, it is nevertheless a surprising result that could benefit from further exploration in the future.

\subsubsection{Analysis of Polarised Sources in Zones 1 and 2}

It would have been acceptable to apply the Duchamp algorithm to these fields to locate polarised sources, as had been done for sources in Stokes-I. Here the low numbers of these meant that it was just as easy to catalogue them by hand, noting down the levels of polarised flux at 

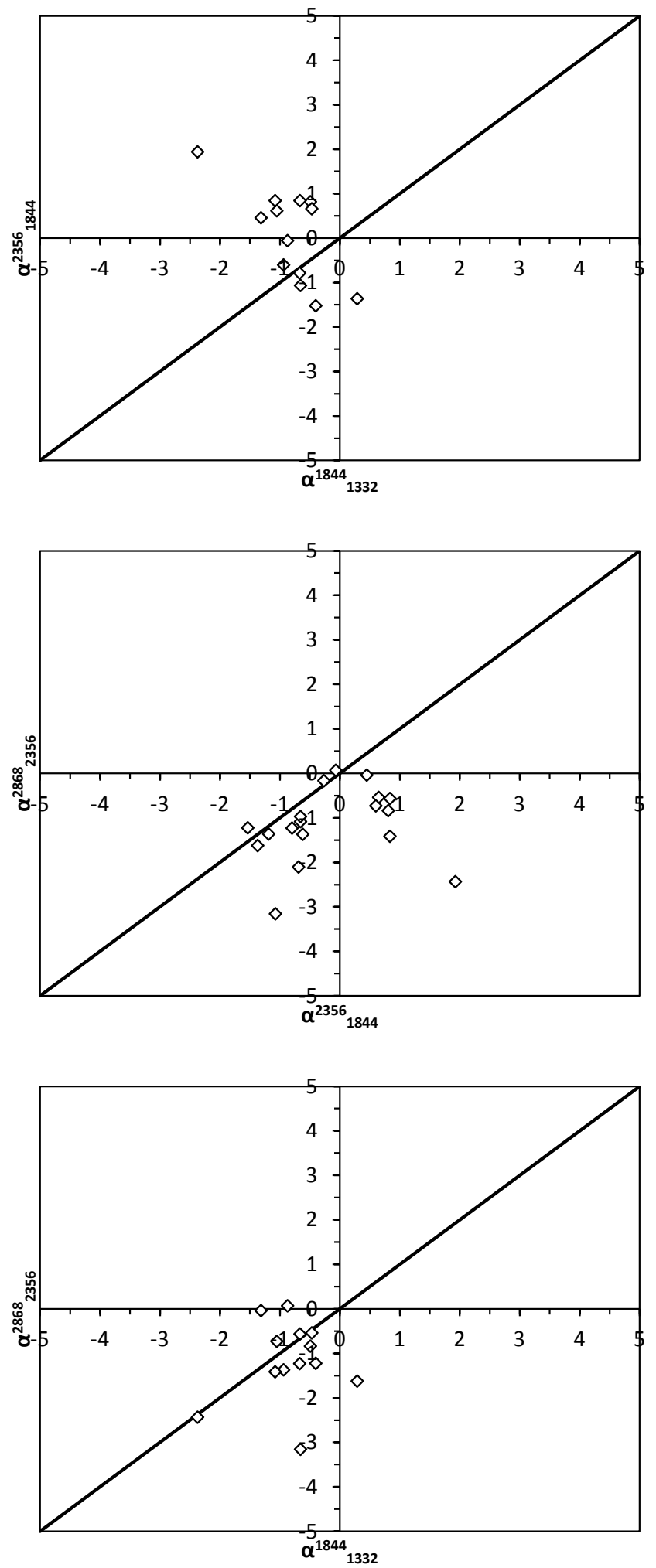

Figure 4.15: Colour-colour plots for spectral index measures of Zone 1 sources, boosted artificially by assuming an additional spectral index of 0.4 , as explained in the text. It can be seen that this attempted correction has done nothing to fix the broad spread of points on either side of the $\mathrm{x}=\mathrm{y}$ line. 


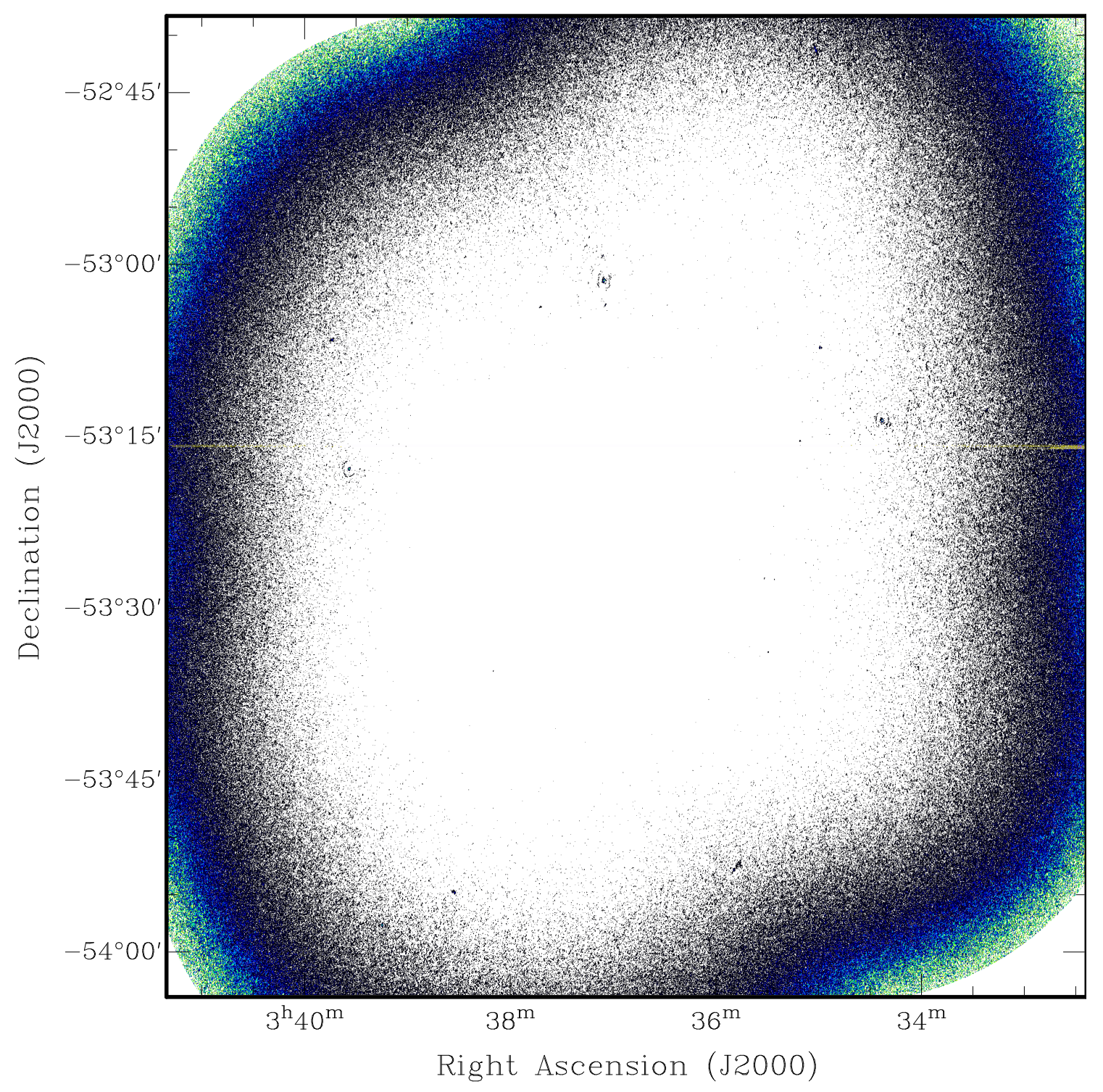

Figure 4.16: Polarisation mosaic of Zone 1 at $1844 \mathrm{MHz}$. Similar to the radio intensity map of Zone 1, this mosaic consisted of 28 pointings observed over 2 sessions in June (14 'core' pointings) \& December (14 'arm' pointings) 2012. Equivalent polarisation mosaics were also made at 1332, 2356 and $2868 \mathrm{MHz}$. As well as these, the single-band data for Zone1(core) were reduced into a 14-pointing mosaic covering that subregion with better sensitivity, allowing the detection of additional sources (5-10, 14 in Table 4.6). 


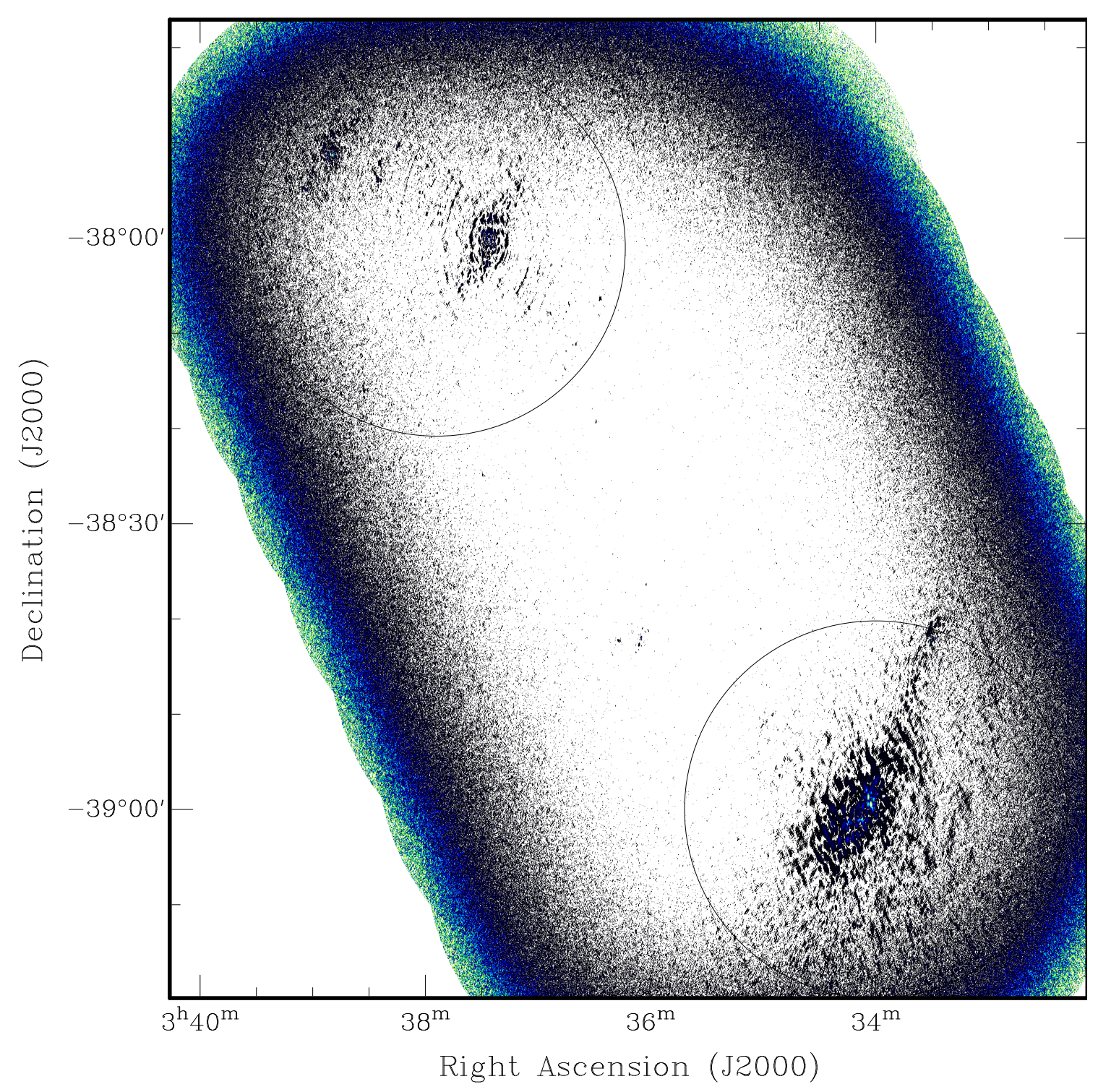

Figure 4.17: Polarisation mosaic of Zone 2 at $1844 \mathrm{MHz}$. The 27 pointings making up this mosaic were all observed during the December 2012 and Jan 2013 session (see Table 3.3). Similar maps were made at 1332, 2356 and $2868 \mathrm{MHz}$. No single-band data existed for this region, as explained in the text. At either end of this filamentary region (top left, bottom right) we're seeing polarised emission within clusters A3145 and A3135 (black circles indicate the positions of these clusters). This appears to be diffuse emission, not associated with discrete sources. 
each frequency. Combining these figures with the Stokes-I fluxes of sources, it was possible to generate profiles of the fractional polarisation of sources across the band. Rather than using the total intensity figures from the Duchamp-generated master catalogues, the values were measured by hand from the images, according to their positions on the polarisation maps. As a result we have measured values for some sources that do not appear at all in the Duchamp catalogues. These are described as $\mathrm{u} / \mathrm{c}$ (uncatalogued) sources and assigned a name based on their lowest-frequency position, while those that do appear are identified by their Duchamp catalogue ID.

Altogether 19 polarised sources were found in Zone 1 ( $\sim 5.7 \%$ of total population), with a further 13 in Zone 2 ( 13\% of total population). Because the Zone1(core) fields were imaged at $2100 \mathrm{MHz}$ before being split into subbands, there existed an extra polarisation image for that subregion and frequency. Some sources in the catalogue were only evident at $2.1 \mathrm{GHz}$. Full details of these sources are presented in Tables 4.6 and 4.7 .

Generally polarised sources exhibit fractional polarisation levels on the order of $10 \%$. Fractional polarisations $\geq 20 \%$ are uncommon. With that in mind, the representation of highly polarised sources in our sample is unusually high - especially for Zone 2 . Source 3 in Zone 1, and Sources 4, 5 and 9 in Zone 2 all show FP $\geq 20 \%$, making $1 / 19$ and $3 / 13$ of the respective polarised populations. All sources with three or more fractional polarisation values have their profiles plotted in Figs 4.18 and 4.19 .

We seem to be seeing some systematic errors in some measures of polarised flux. Several of the plots in Zone 2 (corresponding to sources 2, 5, 8 and 9) show a fractional polarisation level at $1844 \mathrm{MHz}$ significantly lower than expected. In others $(4,7,10,11,13$ in Zone 2; 2 in Zone 1) we see flux measures that drop off at either end of the band, implying an issue with bandpass calibration.

Because the polarised and total fluxes of sources were measured by hand here, this is not an error associated with detection by Duchamp. It is more likely the outlying points in Figs. 4.18 and 4.19 are caused by variations in the polarised sensitivity of ATCA at different frequencies, and for sources in different places relative to pointing centres.

This is an instrumental effect that is known, and had been well studied for the pre-CABB ATCA. The polarisation response profiles for the ATCA at $20 \& 13 \mathrm{~cm}$, drawn from the ATCA observing guide ${ }^{3}$, are shown in Fig. 4.20. At the time of writing a similar calibration of the upgraded instrument - significantly more difficult given the increased bandwidth - has not been undertaken. While the data here suggests inconsistency across the $16 \mathrm{~cm}$ band, without knowing the expected response we cannot perform much in the way of analysis of sources. In any case, polarisation data for our fields were reduced as a sideline to our main (Stokes-I) analysis of the radio populations. The reduction followed the same processes as for the Stokes-I data, and so did not require a large time investment. Proper analysis would require this investment, and isn't crucial to the main research aims of the project.

\footnotetext{
${ }^{3}$ This is the old, pre-CABB guide, available online http://www.atnf.csiro.au/observers/docs/ca_obs_guide/ca_obs_guide.pdf
} 


\begin{tabular}{|c|c|c|c|c|c|c|c|c|c|c|c|c|c|}
\hline \multirow{2}{*}{$\begin{array}{r}\text { Source } \\
\text { No. }\end{array}$} & \multirow{2}{*}{\multicolumn{3}{|c|}{ ID }} & \multirow{2}{*}{\multicolumn{2}{|c|}{$\begin{array}{r}\text { Position } \\
\text { RA } \\
\end{array}$}} & \multirow{2}{*}{$\begin{array}{l}(\mathrm{J} 2000) \\
\text { DEC }\end{array}$} & \multirow{2}{*}{$\begin{array}{l}1332 \\
\text { I (mJy) }\end{array}$} & \multicolumn{2}{|c|}{$\mathrm{MHz}$} & \multicolumn{2}{|r|}{1844} & \multicolumn{2}{|l|}{$\mathrm{MHz}$} \\
\hline & & & & & & & & \multicolumn{2}{|c|}{$\mathrm{P}(\mathrm{mJy})$} & FP & $\mathrm{I}(\mathrm{mJy})$ & $\mathrm{P}(\mathrm{mJy})$ & FP \\
\hline 1 & \multicolumn{3}{|c|}{ M2013 J053.593-53.227 } & \multicolumn{2}{|c|}{$3: 34: 22.1$} & $-53: 13: 33.1$ & 76.4 & \multicolumn{2}{|c|}{5.98} & 0.08 & 83.1 & 4.23 & 0.05 \\
\hline 2 & \multicolumn{3}{|c|}{ M2013 J053.743-53.120 } & \multicolumn{2}{|c|}{$3: 34: 58.4$} & $-53: 07: 08.7$ & 28.8 & \multicolumn{2}{|l|}{0.96} & 0.03 & 21.4 & 0.99 & 0.05 \\
\hline 3 & \multicolumn{3}{|c|}{ M2013 J053.754-53.781 } & \multicolumn{2}{|c|}{$3: 35: 01.0$} & $-52: 41: 03.0$ & 10.4 & \multicolumn{2}{|l|}{4.98} & 0.48 & 15.2 & 7.05 & 0.47 \\
\hline 4 & \multicolumn{3}{|c|}{ u/c J53.775-54.108 } & \multicolumn{2}{|c|}{$3: 35: 05.9$} & $-54: 06: 27.5$ & 105.7 & 7.8 & & 0.07 & 203.8 & 7.87 & 0.04 \\
\hline 5 & M2013 J & $053.794-5$ & 3.256 & $3: 3$ & 10.1 & $-53: 15: 19.4$ & & & & & & & \\
\hline 6 & M2013 J & $053.852-5$ & 3.859 & $3: 3$ & 25.2 & $-53: 53: 26.0$ & & & & & & & \\
\hline 7 & $\mathrm{u} / \mathrm{c} \mathrm{J} 5$ & $3.855-53$. & & $3: 3$ & 25.2 & $-53: 27: 29.5$ & & & & & & & \\
\hline 8 & M2013 J & $053.874-5$ & 3.564 & $3: 3$ & 29.0 & $-53: 33: 46.6$ & & & & & & & \\
\hline 9 & M2013 J & $053.881-5$ & 3.456 & $3: 3$ & 30.5 & $-53: 27: 15.6$ & & & & & & & \\
\hline 10 & M2013 J & $053.946-5$ & 3.433 & $3: 3$ & 45.0 & $-53: 25: 53.2$ & & & & & & & \\
\hline 11 & M2013 J & 053.949-5 & 3.881 & $3: 3$ & 48.8 & $-53: 52: 49.1$ & 36.4 & 1.45 & & 0.04 & 39.2 & 1.58 & 0.04 \\
\hline 12 & M2013 J & $054.271-5$ & 3.023 & $3: 37$ & 04.8 & $-53: 01: 18.0$ & 132.7 & 4.36 & & 0.03 & 140.7 & 4.5 & 0.03 \\
\hline 13 & M2013 J & $054.389-5$ & 2.950 & $3: 37$ & 33.0 & $-52: 55: 34.0$ & 10.6 & 0.55 & & 0.05 & 7.4 & 0.49 & 0.07 \\
\hline 14 & M2013 J & $054.537-5$ & 3.618 & $3: 38$ & 09.0 & $-53: 35: 28.8$ & & & & & & & \\
\hline 15 & M2013 J & $054.635-5$ & 3.914 & $3: 38$ & 31.8 & $-53: 54: 46.9$ & 71.4 & 2.01 & & 0.03 & 60.7 & 2.16 & 0.04 \\
\hline 16 & M2013 J & $054.806-5$ & 3.921 & $3: 3$ & 14.3 & $-53: 57: 37.4$ & 39.1 & 4.92 & & 0.13 & 33.4 & 2.91 & 0.09 \\
\hline 17 & M2013 J & $054.886-5$ & 3.300 & $3: 3$ & 33.5 & $-53: 17: 47.0$ & 58.8 & 5.26 & & 0.09 & 56.9 & 4.14 & 0.07 \\
\hline 18 & M2013 J & $054.928-5$ & 3.145 & $3: 3$ & 43.0 & $-53: 06: 27.6$ & & & & & & & \\
\hline 19 & $\mathrm{u} / \mathrm{c} \mathrm{J} 5$ & $5.051-54$. & & $3: 40$ & 12.2 & $-54: 03: 43.2$ & 42.3 & 5.58 & & 0.13 & 45.2 & 7.65 & 0.17 \\
\hline & Source & 2100 & $\mathrm{MH}_{2}$ & & & 2356 & $\mathrm{MHz}$ & & 286 & & $\mathrm{MHz}$ & & \\
\hline & No. & $\mathrm{I}(\mathrm{mJy})$ & $\mathrm{P}(\mathrm{n}$ & a Jy) & FP & $\mathrm{I}(\mathrm{mJy})$ & $\mathrm{P}(\mathrm{mJy})$ & FP & $\mathrm{I}(\mathrm{n}$ & nJy) & $\mathrm{P}(\mathrm{mJy})$ & FP & \\
\hline & 1 & 92.5 & 3.84 & & 0.04 & 82.0 & 2.43 & 0.03 & 81.8 & & 1.73 & 0.02 & \\
\hline & 2 & 30.4 & 1.37 & & 0.05 & 26.7 & 1.14 & 0.04 & 27.8 & & 0.49 & 0.02 & \\
\hline & 3 & & & & & 19.3 & 3.85 & 0.20 & & & & & \\
\hline & 4 & & & & & & & & & & & & \\
\hline & 5 & 11.0 & 0.47 & & 0.04 & & & & & & & & \\
\hline & 6 & 28.1 & 0.99 & & 0.04 & & & & & & & & \\
\hline & 7 & 2.29 & 0.18 & & 0.08 & & & & & & & & \\
\hline & 8 & 8.83 & 0.31 & & 0.04 & & & & & & & & \\
\hline & 9 & 5.75 & 0.49 & & 0.09 & & & & & & & & \\
\hline & 10 & 2.0 & 0.16 & & 0.08 & & & & & & & & \\
\hline & 11 & 41.8 & 1.25 & & 0.03 & 32.8 & 0.92 & 0.03 & & & & & \\
\hline & 12 & 157 & 5.91 & & 0.04 & 126.1 & 3.15 & 0.02 & 112 & & 2.22 & 0.02 & \\
\hline & 13 & & & & & 8.4 & 0.4 & 0.05 & 0.4 & & 0.24 & 0.59 & \\
\hline & 14 & 9.92 & 0.26 & & 0.03 & & & & & & & & \\
\hline & 15 & 77.4 & 1.97 & & 0.03 & 46.6 & 1.89 & 0.04 & 37.6 & & 1.24 & 0.03 & \\
\hline & 16 & & & & & 29.0 & 1.32 & 0.05 & 22.8 & & 1.06 & 0.05 & \\
\hline & 17 & 54.0 & 2.81 & & 0.05 & 50.3 & 3.62 & 0.07 & & & & & \\
\hline & 18 & 78.9 & 1.59 & & 0.02 & & & & & & & & \\
\hline & 19 & & & & & & & & & & & & \\
\hline
\end{tabular}

Table 4.6: Fractional polarisation details of Zone 1 radio population. Because the first batch of observational data was not split into frequency subbands, we have an additional data point for several sources corresponding to $2.1 \mathrm{GHz}$. As can be seen from the table, a number of polarised sources only appeared in that band. Values of polarised flux and total flux are listed for each source, along with the ratio of the two - the fractional polarisation. This is $\leq 10 \%$ for all sources. Note that the total flux here was measured by hand, as was the case for the polarised flux, rather than use the values from the Duchamp-generated catalogue. Uncertainties in both I and P are on the order of $10 \%$, giving twice this figure for the fractional polarisation. 


\begin{tabular}{|c|c|c|c|c|c|c|c|c|c|}
\hline Sourc & \multirow{2}{*}{\multicolumn{3}{|c|}{ ID }} & Position & \multicolumn{2}{|l|}{ (J2000) } & \multirow{2}{*}{$\begin{array}{l}1332 \\
\mathrm{I}(\mathrm{mJy})\end{array}$} & \multicolumn{2}{|l|}{$\mathrm{MHz}$} \\
\hline $\mathrm{N}$ & & & & RA & DEC & & & $\mathrm{P}(\mathrm{mJy})$ & FP \\
\hline & \multicolumn{3}{|c|}{ u/c J53.367-38.699 } & $3: 33: 28.0$ & \multicolumn{2}{|c|}{$-38: 41: 57.8$} & & \multicolumn{2}{|l|}{1.74} \\
\hline & \multicolumn{3}{|c|}{ u/c J53.373-38.702 } & $3: 33: 29.6$ & \multicolumn{2}{|c|}{$-38: 42: 07.1$} & 41.1 & 2.15 & 0.05 \\
\hline & \multicolumn{3}{|c|}{ u/c J53.374-39.177 } & $3: 33: 29.8$ & \multicolumn{2}{|c|}{$-39: 10: 38.3$} & 30.4 & 1.32 & 0.04 \\
\hline & \multicolumn{3}{|c|}{$\mathrm{u} / \mathrm{c}$ J53.522-39.017 } & $3: 34: 05.2$ & \multicolumn{2}{|c|}{$-39: 01: 01.7$} & 1000 & 162 & 0.16 \\
\hline & \multicolumn{3}{|c|}{$\mathrm{u} / \mathrm{c}$ J53.558-39.054 } & $3: 34: 13.9$ & \multicolumn{2}{|c|}{$-39: 03: 15.0$} & & \multicolumn{2}{|l|}{7.32} \\
\hline & \multicolumn{3}{|c|}{ M2013 J054.018-38.696 } & $3: 36: 04.5$ & \multicolumn{2}{|c|}{$-38: 41: 51.6$} & 15.8 & 1.17 & 0.07 \\
\hline & \multicolumn{3}{|c|}{ M2013 J054.023-38.710 } & $3: 36: 05.5$ & $-38: 42: 3$ & & 6.38 & 0.94 & 0.15 \\
\hline & M201: & J054.164- & 38.182 & $3: 36: 39.3$ & $-38: 11: 0$ & & 19.7 & 0.54 & 0.03 \\
\hline & M201: & J054.356- & 37.994 & $3: 37: 25.4$ & $-37: 59: 5$ & & 57.7 & 15.0 & 0.26 \\
\hline & M201: & J054.362- & 38.026 & $3: 37: 26.8$ & $-38: 02: 3$ & 5.6 & 31.0 & 1.59 & 0.05 \\
\hline & M201: & J054.419- & 38.081 & $3: 37: 40.2$ & $-38: 04: 4$ & 5.8 & 14.3 & 1.11 & 0.08 \\
\hline & $\mathrm{u} / \mathrm{c}$ & J54.706-37 & 854 & $3: 38: 49.4$ & $-37: 51: 1$ & 3.4 & 281 & 9.25 & 0.03 \\
\hline & $\mathrm{u} / \mathrm{c}$ & J54.765-37 & 913 & $3: 39: 03.5$ & $-37: 54: 4$ & 7.5 & 53.0 & 1.51 & 0.03 \\
\hline Source & 1844 & $\mathrm{MHz}$ & & 2356 & $\mathrm{MHz}$ & & 2868 & $\mathrm{MHz}$ & \\
\hline No. & $\mathrm{I}(\mathrm{mJy})$ & $\mathrm{P}(\mathrm{mJy})$ & $\mathrm{FP}$ & $\mathrm{I}(\mathrm{mJy})$ & $\mathrm{P}(\mathrm{mJy})$ & $\mathrm{FP}$ & $\mathrm{I}(\mathrm{mJy})$ & $\mathrm{P}(\mathrm{mJy})$ & FP \\
\hline 1 & 22.0 & 0.68 & 0.03 & 10.9 & 0.40 & 0.04 & 10.1 & 0.33 & 0.03 \\
\hline 2 & 49.0 & 1.02 & 0.02 & 19.1 & 0.74 & 0.04 & 16.1 & 0.53 & 0.03 \\
\hline 3 & 20.0 & 0.72 & 0.04 & & 0.42 & & & 0.34 & \\
\hline 4 & 560 & 191 & 0.34 & 390 & 209 & 0.54 & 570 & 183 & 0.32 \\
\hline 5 & 31.0 & 7.50 & 0.24 & & 3.91 & & 13.0 & 3.33 & 0.26 \\
\hline 6 & 9.67 & 0.65 & 0.07 & 5.00 & 0.38 & 0.08 & 3.00 & 0.32 & 0.11 \\
\hline 7 & 3.10 & 0.53 & 0.17 & 1.80 & 0.28 & 0.16 & 1.60 & 0.17 & 0.11 \\
\hline 8 & 27.3 & 0.49 & 0.02 & 7.90 & 0.27 & 0.03 & 3.60 & 0.13 & 0.04 \\
\hline 9 & 49.9 & 7.02 & 0.14 & 16.8 & 4.78 & 0.28 & 11.1 & 3.35 & 0.30 \\
\hline 10 & 17.2 & 2.32 & 0.13 & 9.50 & 1.70 & 0.18 & 7.40 & 0.81 & 0.11 \\
\hline 11 & 6.29 & 0.75 & 0.12 & 5.30 & 0.62 & 0.12 & 3.30 & 0.29 & 0.09 \\
\hline 12 & 239 & 8.54 & 0.04 & 101 & 6.08 & 0.06 & 125 & 6.49 & 0.05 \\
\hline 13 & 21.2 & 0.99 & 0.05 & 8.90 & 0.55 & 0.06 & 6.20 & 0.19 & 0.03 \\
\hline
\end{tabular}

Table 4.7: Fractional polarisations of radio sources in Zone 2. Values of polarised and total flux are given for the four frequency bands. As before, uncertainties are 10\%, $10 \%$ and $20 \%$ respectively, and flux measurements were measured manually from the appropriate radio images. Here we see a more consistent detection of the same sources across the different bands, than had been the case for Zone 1. Also some sources in Zone 2 have slightly higher fractional polarisations $(\geq 10 \%)$. 

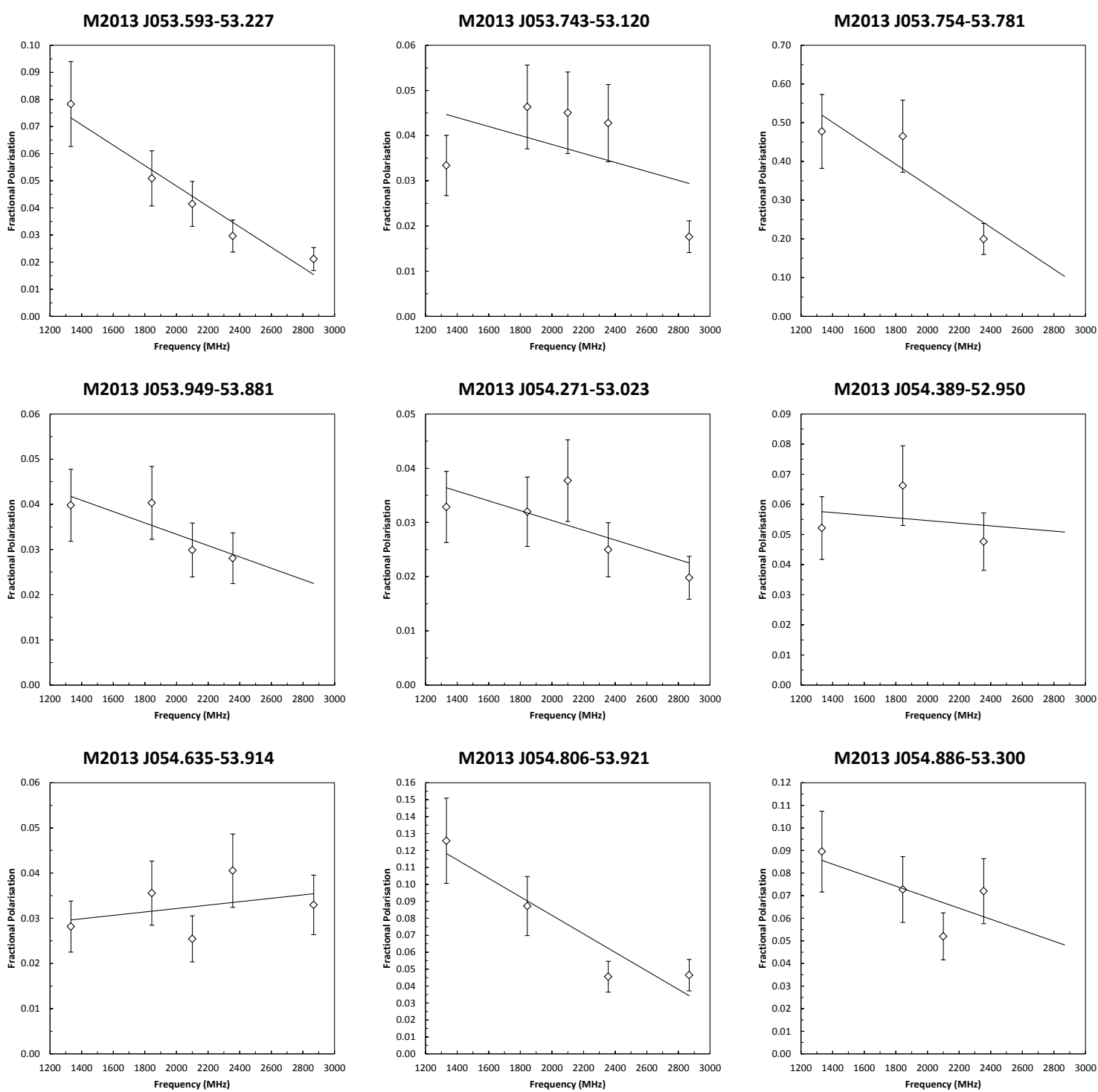

Figure 4.18: Zone 1 Fractional Polarisation plots for all sources with at least three polarised flux measures. Plots correspond to sources in Table $4.6(1,2,3 ; 11,12,13 ; 15,16,17)$. 

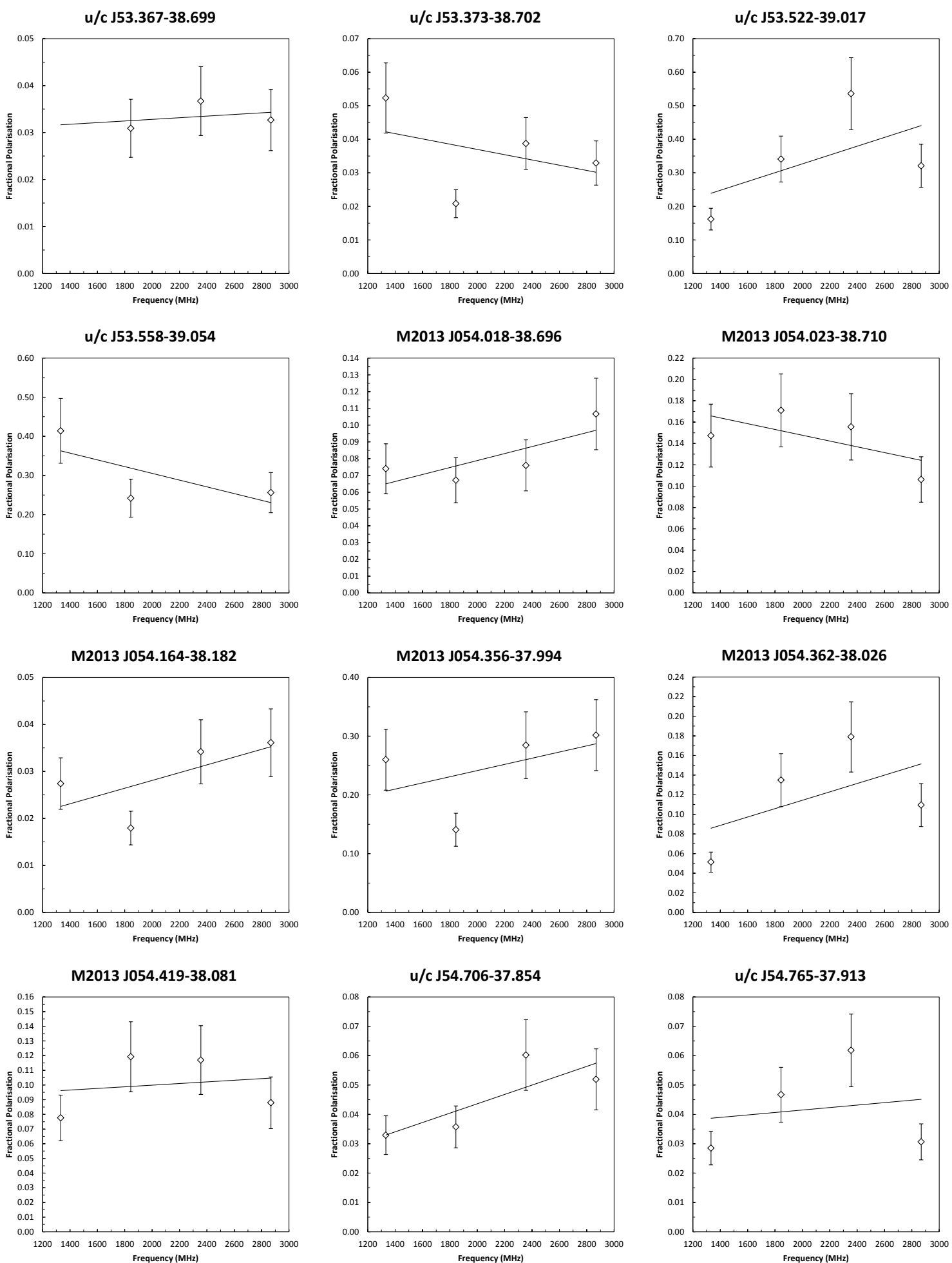

Figure 4.19: Zone 2 Fractional Polarisation plots. u/c indicates that those sources did not appear in the Duchamp-generated master catalogue for Zone 2, and are thus labelled as 'uncatalogued sources' with their lowest-frequency decimal RA and DEC. Plots correspond to sources in Table $4.7(1,2,4 ; 5,6,7 ; 8,9,10 ; 11,12,13)$. 

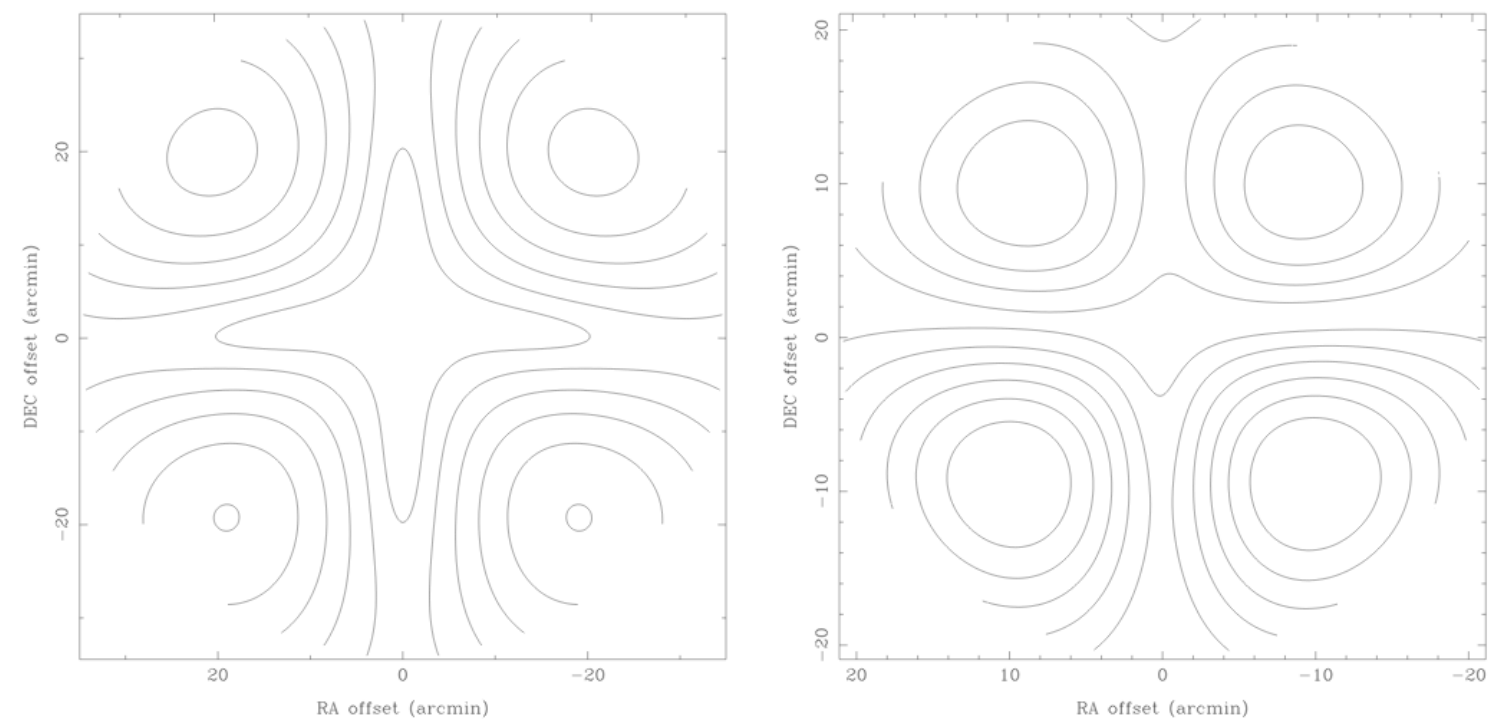

Figure 4.20: The polarised response of pre-CABB ATCA at $1.4 \mathrm{GHz}$ (left) and $2.5 \mathrm{GHz}$ (right) for a simulated source of true flux 1. Clearly here the response varies significantly depending on the location of the source relative to the pointing centre. Contours are 0.001 units for $1.4 \mathrm{GHz}$ and 0.01 units for $2.5 \mathrm{GHz}$. Image from ATCA observing guide (see text). 


\section{Chapter 5}

\section{Discussion}

\subsection{Source Counts in Zones 1 and 2}

In Section 4.1.1 it was seen that while the source count profile of Zone 2 showed strong agreement with the canonical survey of Prandoni et al. (2001), Zone 1 exhibited an excess of radio sources for flux levels $1 \mathrm{mJy} \leq \mathrm{S}_{1.4} \leq 200 \mathrm{mJy}$. Sources above and below this range still showed an excess, but to within the estimated uncertainties the counts were not inconsistent with the expected values here. Additionally, the excess was not limited to only the lower-flux sources, as would likely be the case for false identification of artifacts in the image as sources. Finally, the optical-radio/radio ratio of sources for Zone 1 was consistent with the rate of matching in the Fornax Beta Field survey, suggesting the source counts are probably about right.

To test the significance of the apparent excess in source counts, we applied a KolmogorovSmirnov (KS) test to the Zone 1 and ATESP source count profiles (Prandoni et al. 2001), to see whether these could reasonably have been drawn from similar populations. Because we had only the binned data for the ATESP survey, and not the flux values themselves, we ran the test in two different ways. First, we assumed that fluxes for ATESP sources would be equal to the bin-centre values. The results of this test were $\mathrm{h}=1 ; \mathrm{p}=9.3 \times 10^{-9} ; \mathrm{D}=$ 0.18. This implies the null hypothesis (that the populations are the same) is rejected at the $0.1 \%$ level, so we can be confident that the distributions are different. For the second test, we assumed that the ATESP fluxes were spread evenly across each flux bin. This gave us $\mathrm{h}=1 ; \mathrm{p}=0.022 ; \mathrm{D}=0.087$, meaning that we are less certain here - the null hypothesis is only rejected to the $2.5 \%$ level. As such, we cannot be completely confident that the two populations are significantly different.

It was found (see Section 4.1.4) that the flux calibration for Zone 1 seems to have been applied incorrectly, leading to dubious flux measures for sources in that region. Although this problem seems to have nullified the validity of our spectral index analysis for Zone 1, this cannot be the cause of the excess seen in the source count profile for that population. Based on the quoted flux of 0302-623 from the calibrator database $\left(\mathrm{S}_{2.1} \sim 4.2\right)$ and that of our observations $\left(\mathrm{S}_{2.1}=2.4 \pm 0.2\right)$, it would appear that if anything, we have underestimated the fluxes of sources (This is not necessarily true; source fluxes are known to change over time. 
For this reason this comparison was not used as the basis for flux boosting the population in Section 4.1.4). If this were indeed the case, increasing them would shift the source count profile slightly to the right, increasing the separation between it and the profile of Prandoni et al. (2001). In any case, the effect would be minimised by presenting our source counts on a $\log$ scale, as we have done. Similarly, for the RLF analysis of Zone 1, converting from flux to radio power would have minimised the effect of a slight error in the measured fluxes on the order of the above comparison.

Assuming the flux scaling error for Zone 1 is not large, we can ask the question: why are the source count profiles of Zones 1 and 2 different? This issue can be addressed if we consider the locations of these two filamentary regions relative to the Horologium-Reticulum Supercluster as a whole. Referring again to the map in Fig. 2.1 we see that Zone 1, being defined as the region spanning the rich clusters A3158 and the pair A3125/A3128, lies close to the centre of the supercluster. On the other hand Zone 2, between A3135 and A3145 is much more remote, being only $\sim 3^{\circ}$ from the northernmost extent of the HRS. During the initial field selection this factor was not taken into account. It was assumed that these filaments, which are similar in length and join clusters of comparable richness, would have generally the same properties. However it now seems likely that in Zone 1 we are seeing the effects of a denser environment, providing boosted source counts. Zone 2 is little different to the background. Unfortunately with the available data we were unable to generate an RLF for Zone 2, which would have allowed us to test the comparative likelihood of a galaxy hosting radio emission. Venturi et al. (2000) argued concluded that there was no positive correlation between environment and the ratio of radio to optical flux. However, that study dealt with cluster populations and hence the difference between turbulent and dormant environment ought to be more pronounced than in filaments; it would have made for an interesting comparison.

\subsection{False Detection of Sources with Duchamp}

Much of the analysis of the populations in Zones 1 and 2 was undertaken by means of catalogues generated automatically using the Duchamp source detection algorithm. As with any automated detector, there will inevitably be some degree of false detection of sources from artifacts in the radio image. As a result of the baseline configurations used to observe Zones 1 and 2, radio artifacts were present in the radio maps of both regions. These were unable to be completely removed in the cleaning process. The number of false detections could be greatly reduced by running Duchamp with a higher noise cutoff. This could either be chosen as multiples of $\sigma$ (where $\sigma$ is the noise level determined by Duchamp) or by measuring the noise level by hand and choosing some multiple of that figure explicitly. Trying the former method frequently caused Duchamp to not work correctly - usually by identifying an entire image as 'source' - so the latter was used in this study. The total source counts for Zone 1 were 1239 at $3 \sigma, 540$ at $5 \sigma$ and 332 with a $6 \sigma$ cutoff. What was interesting was that the reduction of spurious sources was not just limited to the low-flux sources; changing the flux cutoff affected the overall rate of detection! There is no reason this should be the case. Furthermore, the quality of the Zone 1 mosaic (Fig. 3.5) was not so bad as to make huge numbers of false detections inevitable. These issues suggest some limitations in the Duchamp algorithm, at least when working with imperfect images. 


\subsection{Radio Luminosity Functions}

The RLF generated for Zone 1 can only loosely be compared to that of Ledlow and Owen (1996), as it covers a radio power regime below the completion limit of that study (see Fig. 4.7). Additionally, in order to match the magnitude limit of Ledlow and Owen we are restricted to only three radio sources with optical counterparts, debasing the quality of our RLF. To work around this we calculated RLFs for Zone 1 and Fornax Beta Field with a fainter magnitude cutoff, allowing more sources to be included in the model (Fig. 4.8). Here we saw that the two RLFs followed a similar trend, with the RLF of Zone 1 extending to a slightly lower radio power than that of Fornax Field. In light of this, comparing the two results as they appear in Fig. 4.7 seems a little out of place. In this figure we see a nice meshing of RLF lines for Fornax Field and A3125, with similar trends seen in A3558 (Venturi et al. 2000) and A3158 (Johnston-Hollitt et al. 2008). The RLF in Zone 1 shows a slight deficit, but the same general placement as that of A3158. This isn't really surprising, as A3158 was one of the clusters that bordered the filamentary region designated as Zone 1. So while the Zone 1 RLF here shows more of a deficit relative to Fornax Field, than in Fig. 4.8, it isn't completely unreasonable. Again here the flux uncertainty associated with Zone 1 may push our RLF to the right, but the effect of this will have been minimised by converting from source flux to radio power, as per Eqn. 1.5.

What is more concerning, is that all five of these RLFs show a significant deficit relative to that of Ledlow and Owen (1996), the canonical example.

A graphical representation of the shortcomings of Ledlow and Owen (1996) is presented in Fig. 5.1. Because generation of a radio luminosity function requires both radio and optical data, the quality of the RLF depends on the sensitivity of these two dimensions. Converting the optical magnitude limit of Ledlow and Owen from $\mathrm{R}_{c}$ to $\mathrm{B}_{J}$ via the treatment in Fukugita et al. (1995), we find that the former probes optical sources down to $\mathrm{B}_{J}=-18.4$, matching these to radio sources as faint as $\mathrm{S}_{1.4}=10 \mathrm{mJy}$. The 'reach' of Ledlow and Owen's RLF is described by the dark blue shaded box in the plot. To put this in perspective, also plotted in Fig. 5.1 are a number of other possible limits. The light blue box describes the reach of the RLF analysis in this study, if adjusted for the redshift in Ledlow and Owen (1996), $z=0.3$. For Zone 1, the sensitivity of the final radio map was around $0.4 \mathrm{mJy}(3 \sigma)$, and combining the Duchamp-generated radio catalogue with optical data from NED and SuperCOSMOS took us down to an absolute magnitude of $\mathrm{B}_{J}=-17.8$. At the actual redshift of the field $(\mathrm{z} \sim 0.06)$, this is much fainter; here $\mathrm{B}_{J}=-14.1$, and we see a significant increase in reach. Additionally the plot includes the quoted optical magnitude limits (at $z=0.3$ ) of the SDSS (Sloan Digital Sky Survey) and LSST (Large Synoptic Survey Telescope, proposed), as well as radio sensitivities of wide field surveys NVSS (NRAO VLA Sky Survey), FIRST (Faint Images of the Radio Sky at Twenty centimetres) and EMU-Wide (Evolutionary Map of the Universe, proposed) (Norris et al. 2013). As can be seen from the plot, even the increase in reach achieved in going from Ledlow and Owen's RLF to that in this study will soon be minor when compared with the possibilities provided by combining upcoming, large scale radio and optical survey data. These large-population-large-area surveys should provide the basis for a more contemporary RLF benchmark. A side effect of this would be that the higher source counts would allow us to consistently represent RLFs on a differential plot, rather than the cumulative RLFs which have been the norm until now. 


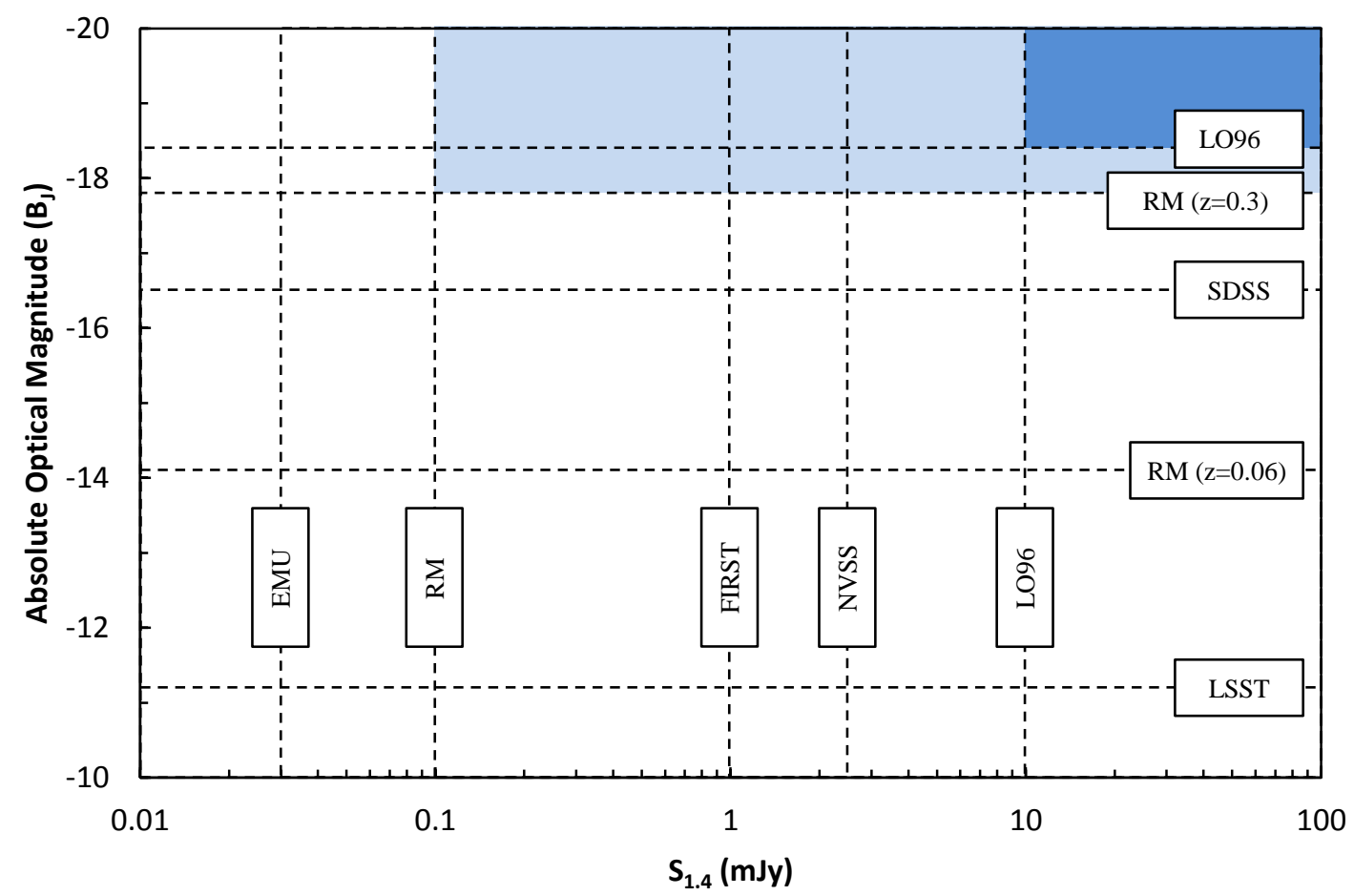

Figure 5.1: This graphic describes the two-dimentional 'reach' of an RLF from a given radio/optical population. The dark blue box represents the canonical RLF from Ledlow and Owen (1996). The light blue box shows the improvement achieved in this study, if we correct the optical magnitudes for the redshift range of Ledlow and Owen, $(z=0.3)$. The main improvement here lies in the radio sensitivity, which is a factor of $10^{2}$ better than the previous. The optical reach of this study at the actual redshift for the HRS $(z=0.06)$ is rather better, but not really a fair comparison. Also included on the plot are a handful of wide-field radio (NVSS, FIRST, EMU-wide) and optical (SDSS, LSST) surveys; some of these are available already, some are yet to be completed. Nevertheless the potential for improvement over Ledlow and Owen (1996) is obvious, making a strong case for the necessity of an updated RLF benchmark that properly probes radio/optical populations into the low-flux-faint-magnitude regime. 
In comparing the RLFs of Zone 1 and Fornax Field with an optical magnitude cutoff of $\mathrm{b}_{J}=22$, it was noted that the former population did indeed seem to exhibit an excess in the low powered (SF-dominated) regime. This was seen in the source counts for that region, but not to a degree beyond the uncertainties. However, comparing the red and blue magnitudes of weak sources (Section 4.9), we saw that five of the six objects in question showed some degree of blue excess, suggesting that the low power overdensity in Zone 1 is associated with a predominantly starforming population.

\subsection{Spectral Indices of Filamentary Populations}

In Section 4.1.4 it was found that the spectral indices of the radio populations in Zones 1 and 2 showed rather different properties. The general trend was that Zone 1 sources exhibited flat or slightly inverted spectra, while sources in Zone 2 demonstrated positive spectral indices (according to the sign convention in Eqn. 2.1) more in keeping with those of classical radio sources. It was noted above that the source counts of Zones 1 and 2 also show differing properties, which can possibly be explained by the locations of the two regions within the HRS; Zone 1 being at the heart of the supercluster and Zone 2 being at its remote northern extent. In light of this, it is not completely surprising to see difference in the types of spectral indices generated for relative populations. The problem is that the situation is contrary to what we should expect. If cluster sources do indeed have steeper indices compared to those in the field (Roland et al. 1985), then we should expect the steeper spectra to be associated with the population of Zone 1. This region was shown to be a denser, and therefore more cluster-like environment. Shallower spectra would more naturally belong to sources in Zone 2 , which displayed a sparser source count profile, and is likely a more relaxed region. But in actuality this is the opposite of what we do see; Zone 2 returned steep spectra, and Zone 1 shallow/inverted spectra. This was initially puzzling, until it was noted that the secondary calibrator for Zone 1, 0302-623, showed a markedly different spectral index across our four bands than the approximate value derived from data on the ATCA calibrator database. In contrast, the two figures agreed for Zone 2 to within uncertainties. Some attempt was made to probe the problem. We boosted the fluxes of sources in the lower three frequency bands by assuming the correctness of the calibrator database-derived index. However, this was still not sufficient to significantly affect the trend seen across the Zone 1 population of inverted spectra across the $2 \mathrm{GHz}$ band of our observations.

\subsubsection{Spectral Indices and CABB}

In the spectral index analysis of Zone 1 and 2 populations, the choice to split the full 2 $\mathrm{GHz}$ bandwidth into four subbands during reduction dictated the manner in which $\alpha$ was generated. To make some investigation of how the $\alpha$ changes across the band, a three-point spectral index is the minimum requirement. Three frequency bands generate a pair of spectral indices that can be compared on a colour-colour plot. Dividing the data into four subbands did allow for an extra point for comparison. It is debatable whether this was useful coming, as it did, at the expense of sensitivity. As was evident in Section 4.1.4 spectral indices could be generated for only a small fraction of each zone catalogue. Clearly here a three-point 
approach would have been desirable, which would allow for a higher number of indices to be generated, helping to address the disparity in spectral index trends between Zones 1 and 2 . However, there simply was not time within the scope of this project to troubleshoot the issue of pre-assigned band subdivision, which would have allowed us to move forward in this area.

While we're on the subject of spectral indices, it is perhaps worth examining the way in which this property is used practically. Elsewhere in this text we have defined the spectral index of a radio source as a continuous function of frequency that describes the decay in the detected flux of the source as we increase the observing frequency. This continuous function has typically been represented as a piecewise set of constant values associated with a given pair of observing frequencies. Adding flux data at additional frequencies can either strengthen the assumption that $\alpha$ remains constant across some frequency range, or else offer some insight to how it varies with frequency.

A prime reason behind the use of this system of spectral indices is the way in which radio observations have been conducted in the past. Until recently radio instruments were limited by narrow observing bandwidths. An example was the pre-CABB version of the ATCA facility, which utilised a maximum bandwidth of $128 \mathrm{MHz}$ for continuum observations. Across such a narrow bandwidth - or rather, considering such a low value of the ratio $\Delta \nu / \nu$ - most observable properties of radio sources could be considered more-or-less constant.

A standard cm-wavelength observing project using pre-CABB might consist of observations at $1.4 \mathrm{GHz}$ and $2.5 \mathrm{GHz}$, possibly with supplementary data available from SUMSS at $843 \mathrm{MHz}$ $(\Delta \nu=3 \mathrm{MHz})$. Maps at these frequencies could provide three effectively-discrete measures of integrated flux for a source, which could then be combined to give a pair of discrete values for the spectral index, $\alpha_{843}^{1.4}$ and $\alpha_{1.4}^{2.5}$.

As became evident during calibration of the observational data in this project, the constancy of observable properties across a bandwidth on the scale of that of CABB is no longer a safe assumption to make. Consider for a moment the frequency difference in the situation above. Very roughly, the frequency increase between MOST at $843 \mathrm{MHz}$ and ATCA at $1.4 \mathrm{GHz}$ is a factor of $\sim 1.7$, and the increase from $1.4 \mathrm{GHz}$ to $2.5 \mathrm{GHz}$ is the same. The $16 \mathrm{~cm}$ band of CABB extends from $1.1 \mathrm{GHz}$ at the low end to $3.1 \mathrm{GHz}$ at the top, a factor of $\sim 2.8$. It is inappropriate, therefore, to use the same method as before and assign a single value of $\alpha$ to the entire $16 \mathrm{~cm}$ band. Additionally, because of the increased sensitivity of CABB it is less likely that useful data will be able to be retrieved from surveys using older instruments like MOST. Our definition of spectral index must take this into account.

If indeed the entire $16 \mathrm{~cm}$ band is being used, it is necessary to divide it into several subbands for the purposes of spectral index analysis. At the time of writing there appears to be no established convention for how best to do this, so any choice of band subdivision is at the discretion of the astronomer reducing the data. If we are treating $\alpha$ as a truly continuous variable, then the more frequencies at which we can make flux measurements, the better. As already mentioned though, more subbands means a lower signal-to-noise ratio in each, and a greater computational load as we duplicate the reduction process ad nauseum. 


\subsection{Linear Mosaic vs Joint Deconvolution}

In this project the 28- and 27-pointing mosaics for Zones 1 and 2 were stitched together after reduction using linmos. For mosaic observation with the pre-CABB ATCA, the observer had the option of combining fields via this method, or by joining at the invert stage (a jointdeconvolution mosaic). At the time of writing, performing joint deconvolution on a wideband data set is not yet possible. The process requires an update to MIRIAD to be able to apply the subband calibrations before deconvolution and then to properly account for the widefield dirty beam. Such an update is currently being developed for MIRIAD, but is not yet complete. Even once that becomes available, in many cases it still may not be possible to combine fields via joint-deconvolution because of the computing power necessary to do so.

The initial reduction of CABB data was performed using MIRIAD via Linux, on a consumerlevel PC. The computing power needed to invert large mosaic maps from CABB data was well outside of its capabilities. Later reduction of this and other data followed the same process by default, even though at that point the reduction had been shifted over onto SPOCK (whose specifications were outlined in Section 1.4.2). Given more time (and the aforementioned MIRIAD update), it would have been interesting to see (i) if that machine could deal with a joint deconvolution on the scale of the mosaic observations in this study, with a sufficiently large image size, and if so (ii) the improvement in the quality of the final radio maps if this method were used. As a minor point, would a joint deconvolution be faster overall? Effectively we are shifting the computational load from time spent cleaning fields to time spent inverting them together. Obviously, this is a topic for consideration at another time, once the appropriate MIRIAD update has been made available. 


\section{Chapter 6}

\section{Conclusions and Further Work}

The findings pertaining to our primary regions of interest, Zones 1 and 2, can be broken down into four main points:

1. We see some evidence of an increase in overall radio activity in filaments, compared to clusters. Comparing the source count profile of Zone 1 with that of Prandoni et al. (2001) there is an excess in source counts. This is within uncertainties for high powered (>200 mJy) sources, while incomplete detection of the low powered population $(<2 \mathrm{mJy})$ makes it unclear whether the source count excess continues or not. Also, results of a KS-test indicated that we cannot be fully confident that the apparent excess is actually significant. It was noted that the flux calibration for Zone 1 was dubious, but the most likely correction would see the whole source count profile move slightly to the right (less consistent with Prandoni et al. 2001). Additionally, small errors here would have little obvious effect on the profile, which is presented on a log scale. The source counts for Zone 2 are consistent with the profile of Prandoni et al..

2. Based on the RLF of Zone 1 we see evidence of an increase in low power log $\mathrm{P}_{1.4}<22$ sources relative to the field RLF drawn from the Fornax Field survey data. This would seem to justify the excess in source counts seen in Zone 1 for $\mathrm{S}_{1.4}<3 \mathrm{mJy}$, which could not be confirmed at that time because of the large uncertainties. The brief investigation of the optical colours of weak sources in Zone 1 suggested that any excess seen in the low power regime is most likely associated with a predominantly starforming population. However, this was based on the results of a very small $\left(\mathrm{n}_{\text {radio }}=6\right)$ sample size, and needs confirmation with a more thorough investigation - ideally through spectroscopic observations to properly diagnose AGN and SF activity through emission and absorption features. Again we see no excess in the starforming population of Zone 2, though without being able to pursue RLF analysis or blue/red magnitude studies, we would be unlikely to do so.

3. The source counts for our two Zones differed from one another, though this was probably the result of cosmic variance as our survey regions were very small $\left(\sim 1^{\circ 2}\right)$, and drawn from different places in the Horologium-Reticulum Supercluster. Zone 2 was near the northern extent of the HRS, wile Zone 1 is near the centre of the supercluster. As 
further studies of filamentary regions are performed and the results stacked together we should expect to see this variance average out, to arrive at a general source count profile for filaments (either in the HRS only, or everywhere).

4. RLF analysis also suffered from the small sample size, though in this case it was the threefold combination of (i) few overall sources (because of the small area covered), (ii) the necessity of coupling the radio catalogue with optical data, reducing the available data pool and (iii) the limitation imposed by adopting an optical magnitude cutoff of $\mathrm{b}_{J}$ $=18.05$ to compare our RLF with the canonical Ledlow and Owen (1996) study, culling the catalogue even further. The first two points can again be rectified by repeating the study for a number of other filamentary regions, stacking the results. However, as was shown in Section 5.3 the RLF of Ledlow and Owen is now outdated and its use as a primary comparison seriously restricts the effectiveness of RLF analysis for lowbrightness-low-flux populations, as ours is. The need for a new RLF benchmark that probes the low power regime is ever-increasing.

While the spectral indices derived for the sources in Zone 2 were consistent with expected values for a typical radio population, those of Zone 1 were notably different. The bulk of these were inverted, and many showed much greater variation across the $2 \mathrm{GHz}$ bandwidth of the observations than the Zone 2 population. This was thought to have stemmed from an error in calibration. However, an attempted first-order correction using an additional spectral index of 0.4 to boost the fluxes of sources relative to their $2868 \mathrm{MHz}$ fluxes, was unsuccessful. The resulting spectra were still significantly inverted and variable. To suitably address the problem it would be necessary to return to the initial calibration of the Zone 1 data, and then proceed with the remainder of reduction. Although much of the process (e.g. flagging) would be far quicker than the first time around, such a treatment was unable to be performed within the timescale of this study.

As was mentioned during the analysis of polarised sources in Zones 1 and 2, a benchmarking study of the polarised response of CABB across the $2.1 \mathrm{GHz}$ is yet to be performed. Such a study is necessary before we can begin to draw conclusions from the fractional polarisation profiles of sources generated in that part of this study.

In the initial selection of filamentary zones of interest within the HRS is was noted that both Zone 1 and Zone 2 had observations covering the adjoining clusters. Although it was outside the scope of this study, it would be possible to combine these previous data - which have been reduced by the Radio Astronomy Group at Victoria University of Wellington - with our filamentary mosaics. This could potentially offer a laboratory to study physical effects on galaxies as a function of distance from cluster centres, covering a large proximity range. This could provide the basis for a short-term research project at honours-level or similar.

Although probably less useful, there were also several data sets pertaining to Filament 17 that were not reduced in this project. Because we were interested in distant point sources, the decision was made to reduce only those data with $6 \mathrm{~km}$ baselines for maximum resolution. Much of our data for Zones 1 and 2 actually used 1.5km baselines (either from the original observations, or from the removal of Antenna 6 visibilities during reduction), with reasonable success. All the sources found in Filament 17 were foreground sources, not in the HRS, and most were extended. It would be necessary to estimate the increase in sensitivity from 
combining $1.5 \mathrm{~km}$ data to the $6 \mathrm{~km}$ data already reduced, and compare this with the depth of our other observations - particularly Zone 1, where the SF regime was sufficiently probed.

An area only briefly touched on in this study was colour analysis of weak sources. A firstorder analysis was attempted with the SuperCOSMOS catalogue for Zone 1, comparing the blue and red magnitude data for sources in the region. The low number of optical/radio correlations further limited to optical magnitudes $\mathrm{b}_{J} \leq 20$ left us with only six sources. A better course of action would be to perform follow-up spectroscopic observations for sources in Zones 1 and 2 using a suitable instrument like AAOmega (Anglo-Australian Observatory).

Finally, this study focused on continuum radio observations. Additional insight could be had from pursuing spectral line observations of the same regions. SF galaxies are known to host an excess of molecular gas, while this has been exhausted in AGNs. Probing the signatures of prominent species (e.g. HI, CO) could provide a complementary indicator of the kinds of radio galaxies found in filaments in the HRS.

This thesis represents a brief excursion into observation and analysis of filamentary radio populations using a current-generation telescope. It is by no means exhaustive, and several avenues remain open, inviting further study. In Section 5.3 it was noted that a number new radio instruments will be coming online over the next $\sim 10$ years. Combining observations from these telescopes with new optical surveys has the potential to unlock previously unexplored parameter space (see Fig. 5.1), allowing astronomers to study the way that environment affects radio emission over cosmic timescales. As a result we expect to see growing interest in filamentary populations from the radio community in the coming years.

\section{Acknowledgements}

The author wishes to acknowledge the assistance of the following people and organisations:

- Tiziana Venturi and Istituto di Radioastronomia at CNR Area della Ricerca, Bologna for accommodating me while I brought this thesis towards completion.

- The ATCA staff, who allowed me to undertake my first set of observations during scheduled green time.

- The International Astronomical Union, and J L Stewart for the award of generous scholarships allowing me to attend the XXVIII IAU General Assembly 2012 in Beijing.

- CSIRO, for assisting my attendance at the 2012 Radio Astronomy School.

- The Radio Astronomy group at Victoria University of Wellington, but especially Melanie JohnstonHollitt, Luke Hindson, Siamak Dehghan and Luke Pratley for all their help along the way.

The Australia Telescope Compact Array is operated by the CSIRO Australia Telescope National Facility which is funded by the Commonwealth of Australia for operation as a National Facility managed by CSIRO.

This paper includes archived data obtained through the Australia Telescope Online Archive (http://atoa.atnf.csiro.au).

The Digitized Sky Surveys were produced at the Space Telescope Science Institute under U.S. Government grant NAG W-2166. The images of these surveys are based on photographic data obtained using the Oschin Schmidt Telescope on Palomar Mountain and the UK Schmidt Telescope. The plates were processed into the present compressed digital form with the permission of these institutions.

This research made use of the Duchamp source finder, produced at the Australia Telescope National Facility, CSIRO, by M. Whiting. 
Miriad software and documentation are distributed under the GNU General Public License from the Miriad ftp area, ftp://ftp.atnf.csiro.au/pub/software/miriad/.

This research has made use of the NASA/IPAC Extragalactic Database (NED) which is operated by the Jet Propulsion Laboratory, California Institute of Technology, under contract with the National Aeronautics and Space Administration.

This research has made use of data obtained from the SuperCOSMOS Science Archive, prepared and hosted by the Wide Field Astronomy Unit, Institute for Astronomy, University of Edinburgh, which is funded by the UK Science and Technology Facilities Council.

TOPCAT is written in pure Java and available under the GNU General Public Licence. It has been developed mostly in the UK within various UK and Euro-VO projects (Starlink, AstroGrid, VOTech, AIDA, GAVO) and under PPARC and STFC grants. 


\section{Appendices}

\section{Appendix 1: Example Reduction Scripts}

All examples will deal with a simplified mosaic-pointing consisting of four fields, each containing a single frequency band centred at $2.1 \mathrm{GHz}$. Expansion to full data complement is trivial by means of simple loops within the script.

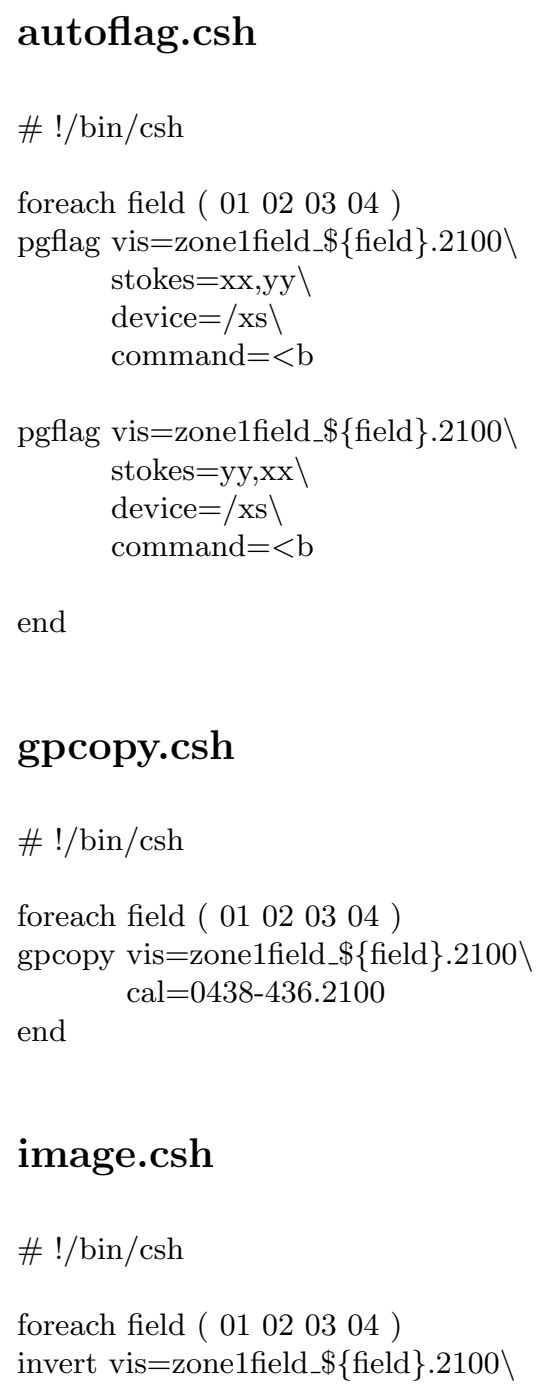


map=zone1field_ $\$\{$ field $\} .2100 . i m \backslash$

beam $=$ zone1field $\_\$\{$ field $\} .2100 . i b \backslash$

imsize $=4096 \backslash$

cell $=2.4 \backslash$

stokes $=\mathrm{i} \backslash$

robust $=0.5$

options $=$ double, mfs, mosaic, $\mathrm{sdb}$

mfclean map=zone1field $\_\$$ field $\} .2100$. im $\backslash$ beam $=$ zone1field $\_\{\text {field }\} .2100 . i b \backslash$ out=zone1field_\$\{field $\} .2100$. icln $\backslash$ cutoff $=1.5 \mathrm{e}-4 \backslash$ niters $=100000 \backslash$

region=@zone1field_\$ffield $\}$.region

restor model $=$ zone1field_ $\$\{$ field $\} .2100 . i c \ln \backslash$ map $=$ zone1field_ $\$\{$ field $\} .2100 . \mathrm{im} \backslash$ beam $=$ zone1field__ffield $\} .2100 . i b \backslash$ out=zone1field $\_\$$ field $\} .2100$.iimag

end 


\section{Bibliography}

G. O. Abell, H. G. Corwin, Jr., and R. P. Olowin. A catalog of rich clusters of galaxies. ApJS, 70:1-138, May 1989. doi: $10.1086 / 191333$.

M. A. Aragón-Calvo, R. van de Weygaert, and B. J. T. Jones. Multiscale phenomenology of the cosmic web. MNRAS, 408:2163-2187, November 2010. doi: 10.1111/j.1365-2966.2010.17263.x.

S. Bardelli, A. Pisani, M. Ramella, E. Zucca, and G. Zamorani. A substructure analysis of the A3558 cluster complex. MNRAS, 300:589-598, October 1998. doi: 10.1046/j.1365-8711.1998.01930.x.

J. R. Bond, L. Kofman, and D. Pogosyan. How filaments of galaxies are woven into the cosmic web. Nature, 380:603-606, April 1996. doi: 10.1038/380603a0.

H. Butcher and A. Oemler, Jr. The evolution of galaxies in clusters. V - A study of populations since Z approximately equal to 0.5 . ApJ, 285:426-438, October 1984. doi: 10.1086/162519.

N. Caldwell and J. A. Rose. The Butcher-Oemler Effect at Low Redshift: Spectroscopy of Five Nearby Clusters of Galaxies. AJ, 113:492, February 1997. doi: 10.1086/118271.

C. L. Carilli and P. D. Barthel. Cygnus A. A\&ARv, 7:1-54, 1996. doi: 10.1007/s001590050001.

M. Colless, G. Dalton, S. Maddox, W. Sutherland, P. Norberg, S. Cole, J. Bland-Hawthorn, T. Bridges, R. Cannon, C. Collins, W. Couch, N. Cross, K. Deeley, R. De Propris, S. P. Driver, G. Efstathiou, R. S. Ellis, C. S. Frenk, K. Glazebrook, C. Jackson, O. Lahav, I. Lewis, S. Lumsden, D. Madgwick, J. A. Peacock, B. A. Peterson, I. Price, M. Seaborne, and K. Taylor. The 2dF Galaxy Redshift Survey: spectra and redshifts. MNRAS, 328:1039-1063, December 2001. doi: 10.1046/j.1365-8711.2001.04902.x.

J. J. Condon. Radio emission from normal galaxies. ARAA, 30:575-611, 1992 . doi: 10.1146/annurev.aa.30.090192.003043.

J. J. Condon, W. D. Cotton, and J. J. Broderick. Radio Sources and Star Formation in the Local Universe. AJ, 124:675-689, August 2002. doi: 10.1086/341650.

G. de Zotti, M. Massardi, M. Negrello, and J. Wall. Radio and millimeter continuum surveys and their astrophysical implications. A\&ARv, 18:1-65, February 2010. doi: 10.1007/s00159-009-0026-0.

J. P. Dietrich, P. Schneider, D. Clowe, E. Romano-Díaz, and J. Kerp. Weak lensing study of dark matter filaments and application to the binary cluster A 222 and A 223. A\&\&A, 440:453-471, September 2005. doi: 10.1051/0004-6361:20041523.

A. Dressler and J. E. Gunn. Spectroscopy of galaxies in distant clusters. II - The population of the 3C 295 cluster. ApJ, 270:7-19, July 1983. doi: 10.1086/161093.

F. Durret, G. B. Lima Neto, W. Forman, and E. Churazov. An XMM-Newton view of the extended "filament" near the cluster of galaxies Abell 85. A\&\&A, 403:L29-L32, May 2003. doi: 10.1051/0004-6361:20030424.

D. Fadda, A. Biviano, F. R. Marleau, L. J. Storrie-Lombardi, and F. Durret. Starburst Galaxies in Clusterfeeding Filaments Unveiled by Spitzer. ApJ, 672:L9-L12, January 2008. doi: 10.1086/526457. 
M. C. Fleenor. Morphology and large-scale structure within the Horologium-Reticulum supercluster of galaxies. $\mathrm{PhD}$ thesis, The University of North Carolina at Chapel Hill, 2006.

M. C. Fleenor, J. A. Rose, W. A. Christiansen, R. W. Hunstead, M. Johnston-Hollitt, M. J. Drinkwater, and W. Saunders. Large-Scale Velocity Structures in the Horologium-Reticulum Supercluster. AJ, 130:957-967, September 2005. doi: 10.1086/431972.

M. Fukugita, K. Shimasaku, and T. Ichikawa. Galaxy Colors in Various Photometric Band Systems. PASP, 107:945, October 1995. doi: 10.1086/133643.

S. Giacintucci, T. Venturi, S. Bardelli, D. Dallacasa, and E. Zucca. AGN and starburst radio activity in the A3558 cluster complex. A\&SA, 419:71-87, May 2004. doi: 10.1051/0004-6361:20040071.

M. D. Gladders and H. K. C. Yee. A New Method For Galaxy Cluster Detection. I. The Algorithm. AJ, 120: 2148-2162, October 2000. doi: 10.1086/301557.

J. E. Gunn and J. R. Gott, III. On the Infall of Matter Into Clusters of Galaxies and Some Effects on Their Evolution. ApJ, 176:1, August 1972. doi: 10.1086/151605.

C. P. Haines, G. Busarello, P. Merluzzi, R. J. Smith, S. Raychaudhury, A. Mercurio, and G. P. Smith. ACCESS - III. The nature of star formation in the Shapley supercluster. MNRAS, 412:145-160, March 2011. doi: 10.1111/j.1365-2966.2010.17892.x.

A. M. Hopkins, C. J. Miller, R. C. Nichol, A. J. Connolly, M. Bernardi, P. L. Gómez, T. Goto, C. A. Tremonti, J. Brinkmann, Ž. Ivezić, and D. Q. Lamb. Star Formation Rate Indicators in the Sloan Digital Sky Survey. ApJ, 599:971-991, December 2003. doi: 10.1086/379608.

A. Jenkins, C. S. Frenk, F. R. Pearce, P. A. Thomas, J. M. Colberg, S. D. M. White, H. M. P. Couchman, J. A. Peacock, G. Efstathiou, and A. H. Nelson. Evolution of Structure in Cold Dark Matter Universes. $A p J, 499: 20$, May 1998. doi: 10.1086/305615.

S. Johnston, R. Taylor, M. Bailes, N. Bartel, C. Baugh, M. Bietenholz, C. Blake, R. Braun, J. Brown, S. Chatterjee, J. Darling, A. Deller, R. Dodson, P. Edwards, R. Ekers, S. Ellingsen, I. Feain, B. Gaensler, M. Haverkorn, G. Hobbs, A. Hopkins, C. Jackson, C. James, G. Joncas, V. Kaspi, V. Kilborn, B. Koribalski, R. Kothes, T. Landecker, A. Lenc, J. Lovell, J.-P. Macquart, R. Manchester, D. Matthews, N. McClure-Griffiths, R. Norris, U.-L. Pen, C. Phillips, C. Power, R. Protheroe, E. Sadler, B. Schmidt, I. Stairs, L. Staveley-Smith, J. Stil, S. Tingay, A. Tzioumis, M. Walker, J. Wall, and M. Wolleben. Science with ASKAP. The Australian square-kilometre-array pathfinder. ExA, 22:151-273, December 2008. doi: 10.1007/s10686-008-9124-7.

M. Johnston-Hollitt, M. Sato, J. A. Gill, M. C. Fleenor, and A.-M. Brick. Radio observations of the Horologium-Reticulum supercluster - I. A3158: excess star-forming galaxies in a merging cluster? MNRAS, 390:289-303, October 2008. doi: 10.1111/j.1365-2966.2008.13730.x.

A. Kull and H. Böhringer. Detection of filamentary X-ray structure in the core of the Shapley supercluster. A\&A, 341:23-28, January 1999.

M. J. Ledlow and F. N. Owen. 20 CM VLA Survey of Abell Clusters of Galaxies. VI. Radio/Optical Luminosity Functions. AJ, 112:9, July 1996. doi: 10.1086/117985.

M. Y. Mao, M. Johnston-Hollitt, J. B. Stevens, and S. J. Wotherspoon. Head-tail Galaxies: beacons of highdensity regions in clusters. MNRAS, 392:1070-1079, January 2009. doi: 10.1111/j.1365-2966.2008.14141.x.

T. Mauch and E. M. Sadler. Radio sources in the 6dFGS: local luminosity functions at $1.4 \mathrm{GHz}$ for star-forming galaxies and radio-loud AGN. MNRAS, 375:931-950, March 2007. doi: 10.1111/j.1365-2966.2006.11353.x.

T. Mauch, T. Murphy, H. J. Buttery, J. Curran, R. W. Hunstead, B. Piestrzynski, J. G. Robertson, and E. M. Sadler. SUMSS: a wide-field radio imaging survey of the southern sky - II. The source catalogue. MNRAS, 342:1117-1130, July 2003. doi: 10.1046/j.1365-8711.2003.06605.x.

G. K. Miley, G. C. Perola, P. C. van der Kruit, and H. van der Laan. Active Galaxies with Radio Trails in Clusters. Nature, 237:269-272, June 1972. doi: 10.1038/237269a0. 
N. A. Miller and F. N. Owen. Evolution of Star-forming and Active Galaxies in Nearby Clusters. AJ, 124: 2453-2470, November 2002. doi: 10.1086/343837.

N. A. Miller and F. N. Owen. Abell 2255: Increased Star Formation and AGN Activity in a Cluster-Cluster Merger. AJ, 125:2427-2446, May 2003. doi: 10.1086/374767.

G. E. Morrison and F. N. Owen. Radio-selected Galaxies in Very Rich Clusters at z $\leq 0.25$. II. Radio Properties and Analysis. AJ, 125:506-513, February 2003. doi: 10.1086/346096.

T. Muxlow, R. J. Beswick, A. M. S. Richards, and H. J. Thrall. Starburst galaxies. In Proceedings of the 8th European VLBI Network Symposium, 2006.

R. P. Norris, A. M. Hopkins, J. Afonso, S. Brown, J. J. Condon, L. Dunne, I. Feain, R. Hollow, M. Jarvis, M. Johnston-Hollitt, E. Lenc, E. Middelberg, P. Padovani, I. Prandoni, L. Rudnick, N. Seymour, G. Umana, H. Andernach, D. M. Alexander, P. N. Appleton, D. Bacon, J. Banfield, W. Becker, M. J. I. Brown, P. Ciliegi, C. Jackson, S. Eales, A. C. Edge, B. M. Gaensler, G. Giovannini, C. A. Hales, P. Hancock, M. T. Huynh, E. Ibar, R. J. Ivison, R. Kennicutt, A. E. Kimball, A. M. Koekemoer, B. S. Koribalski, Á. R. López-Sánchez, M. Y. Mao, T. Murphy, H. Messias, K. A. Pimbblet, A. Raccanelli, K. E. Randall, T. H. Reiprich, I. G. Roseboom, H. Röttgering, D. J. Saikia, R. G. Sharp, O. B. Slee, I. Smail, M. A. Thompson, J. S. Urquhart, J. V. Wall, and G.-B. Zhao. EMU: Evolutionary Map of the Universe. PASA, 28:215-248, August 2011. doi: $10.1071 / \mathrm{AS} 11021$.

R. P. Norris, J. Afonso, D. Bacon, R. Beck, M. Bell, R. J. Beswick, P. Best, S. Bhatnagar, A. Bonafede, G. Brunetti, T. Budavári, R. Cassano, J. J. Condon, C. Cress, A. Dabbech, I. Feain, R. Fender, C. Ferrari, B. M. Gaensler, G. Giovannini, M. Haverkorn, G. Heald, K. Van der Heyden, A. M. Hopkins, M. Jarvis, M. Johnston-Hollitt, R. Kothes, H. Van Langevelde, J. Lazio, M. Y. Mao, A. Martínez-Sansigre, D. Mary, K. Mcalpine, E. Middelberg, E. Murphy, P. Padovani, Z. Paragi, I. Prandoni, A. Raccanelli, E. Rigby, I. G. Roseboom, H. Röttgering, J. Sabater, M. Salvato, A. M. M. Scaife, R. Schilizzi, N. Seymour, D. J. B. Smith, G. Umana, G.-B. Zhao, and P.-C. Zinn. Radio Continuum Surveys with Square Kilometre Array Pathfinders. PASA, 30:e020, March 2013. doi: 10.1017/pas.2012.020.

F. N. Owen, M. J. Ledlow, W. C. Keel, and G. E. Morrison. Cluster Mergers as Triggers of Star Formation and Radio Emission: A Comparative Study of the Rich Clusters Abell 2125 and 2645. AJ, 118:633-644, August 1999. doi: 10.1086/300974.

Planck Collaboration, P. A. R. Ade, N. Aghanim, M. Arnaud, M. Ashdown, F. Atrio-Barandela, J. Aumont, C. Baccigalupi, A. Balbi, A. J. Banday, and et al. Planck intermediate results. VIII. Filaments between interacting clusters. A\&SA, 550:A134, February 2013. doi: 10.1051/0004-6361/201220194.

S. C. Porter, S. Raychaudhury, K. A. Pimbblet, and M. J. Drinkwater. Star formation in galaxies falling into clusters along supercluster-scale filaments. MNRAS, 388:1152-1160, August 2008. doi: 10.1111/j.13652966.2008.13388.x.

I. Prandoni, L. Gregorini, P. Parma, H. R. de Ruiter, G. Vettolani, M. H. Wieringa, and R. D. Ekers. The ATESP radio survey. III. Source counts. AESA, 365:392-399, January 2001. doi: 10.1051/00046361:20000142.

L. Pratley, M. Johnston-Hollitt, S. Dehghan, and M. Sun. Using head-tail galaxies to constrain the intracluster magnetic field: an in-depth study of PKS J0334-3900. MNRAS, 432:243-257, June 2013. doi: $10.1093 / \mathrm{mnras} / \mathrm{stt} 448$.

V. Quilis, B. Moore, and R. Bower. Gone with the Wind: The Origin of S0 Galaxies in Clusters. Science, 288:1617-1620, June 2000. doi: 10.1126/science.288.5471.1617.

G. H. Rieke, A. Alonso-Herrero, B. J. Weiner, P. G. Pérez-González, M. Blaylock, J. L. Donley, and D. Marcillac. Determining Star Formation Rates for Infrared Galaxies. ApJ, 692:556-573, February 2009. doi: 10.1088/0004-637X/692/1/556.

K. Roettiger, J. O. Burns, and C. Loken. The Observational Consequences of Merging Clusters of Galaxies. ApJ, 473:651, December 1996. doi: 10.1086/178179. 
J. Roland, R. J. Hanisch, P. Veron, and E. Fomalont. WSRT and VLA observations of very steep spectrum radio galaxies in clusters. A\& $A, 148: 323-334$, July 1985.

L. Rudnick and F. N. Owen. Head-tail radio sources in clusters of galaxies. ApJL, 203:L107-L111, February 1976. doi: $10.1086 / 182030$.

R. J. Sault and M. H. Wieringa. Multi-frequency synthesis techniques in radio interferometric imaging. $A \mathscr{E} A S$, 108:585-594, December 1994.

R. J. Sault, P. J. Teuben, and M. C. H. Wright. A Retrospective View of MIRIAD. In R. A. Shaw, H. E. Payne, \& J. J. E. Hayes, editor, Astronomical Data Analysis Software and Systems IV, volume 77 of Astronomical Society of the Pacific Conference Series, pages 433-+, 1995.

C. Scharf, M. Donahue, G. M. Voit, P. Rosati, and M. Postman. Evidence for X-Ray Emission from a Large-Scale Filament of Galaxies? ApJ, 528:L73-L76, January 2000. doi: 10.1086/312435.

A. R. Taylor and E. R. Seaquist. A deep VLA survey of a galactic field - A search for stellar radio emission. $A J$, 90:2049-2054, October 1985. doi: 10.1086/113911.

S. Tingay, R. Goeke, J. N. Hewitt, E. Morgan, R. A. Remillard, C. L. Williams, J. D. Bowman, E. Emrich, S. M. Ord, T. Booler, B. Crosse, D. Pallot, W. Arcus, T. Colegate, P. J. Hall, D. Herne, M. J. Lynch, F. Schlagenhaufer, S. Tremblay, R. B. Wayth, M. Waterson, D. A. Mitchell, R. J. Sault, R. L. Webster, J. S. B. Wyithe, M. F. Morales, B. J. Hazelton, A. Wicenec, A. Williams, D. Barnes, G. Bernardi, L. J. Greenhill, J. C. Kasper, F. Briggs, B. McKinley, J. D. Bunton, L. deSouza, R. Koenig, J. Pathikulangara, J. Stevens, R. J. Cappallo, B. E. Corey, B. B. Kincaid, E. Kratzenberg, C. J. Lonsdale, S. R. McWhirter, A. E. E. Rogers, J. E. Salah, A. R. Whitney, A. Deshpande, T. Prabu, A. Roshi, N. Udaya-Shankar, K. S. Srivani, R. Subrahmanyan, B. M. Gaensler, M. Johnston-Hollitt, D. L. Kaplan, and D. Oberoi. Realisation of a low frequency SKA Precursor: The Murchison Widefield Array. In Resolving The Sky Radio Interferometry: Past, Present and Future, 2012.

T. Venturi, S. Bardelli, R. Morganti, and R. W. Hunstead. Radio properties of the Shapley Concentration I. The Abell cluster A3556. MNRAS, 285:898-912, March 1997.

T. Venturi, S. Bardelli, R. Morganti, and R. W. Hunstead. Radio properties of the Shapley Concentration - III. Merging clusters in the A3558 complex. MNRAS, 314:594-610, May 2000. doi: 10.1046/j.13658711.2000.03403.x.

M. T. Whiting. DUCHAMP: a 3D source finder for spectral-line data. MNRAS, 421:3242-3256, April 2012. doi: $10.1111 /$ j.1365-2966.2012.20548.x.

W. E. Wilson, R. H. Ferris, P. Axtens, A. Brown, E. Davis, G. Hampson, M. Leach, P. Roberts, S. Saunders, B. S. Koribalski, J. L. Caswell, E. Lenc, J. Stevens, M. A. Voronkov, M. H. Wieringa, K. Brooks, P. G. Edwards, R. D. Ekers, B. Emonts, L. Hindson, S. Johnston, S. T. Maddison, E. K. Mahony, S. S. Malu, M. Massardi, M. Y. Mao, D. McConnell, R. P. Norris, D. Schnitzeler, R. Subrahmanyan, J. S. Urquhart, M. A. Thompson, and R. M. Wark. The Australia Telescope Compact Array Broad-band Backend: description and first results. MNRAS, 416:832-856, September 2011. doi: 10.1111/j.1365-2966.2011.19054.x. 